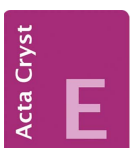

CRYSTALLOGRAPHIC COMMUNICATIONS

ISSN 2056-9890

Received 27 June 2018

Accepted 6 August 2018

Edited by K. Fejfarova, Institute of Biotechnology CAS, Czech Republic

Keywords: $\mathrm{RbGa}\left(\mathrm{HAsO}_{4}\right)_{2} ; \mathrm{RbGa}_{2} \mathrm{As}\left(\mathrm{HAsO}_{4}\right)_{6}$; $\mathrm{AsO}_{6}$; arsenate; hydrogenarsenate; $\mathrm{AsO}_{6}$ octahedra; crystal structure.

CCDC references: 1860366; 1860365

Supporting information: this article has supporting information at journals.iucr.org/e

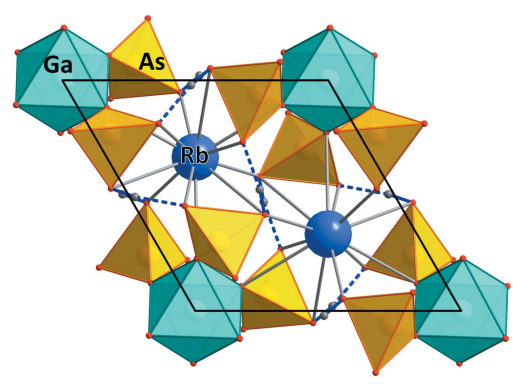

OPEN $\odot$ ACCESS

\section{Two new $\mathrm{Rb}-\mathrm{Ga}$ arsenates: $\mathrm{RbGa}\left(\mathrm{HAsO}_{4}\right)_{2}$ and $\mathrm{RbGa}_{2} \mathrm{As}\left(\mathrm{HAsO}_{4}\right)_{6}$}

\author{
Karolina Schwendtner $^{\mathrm{a} *}$ and Uwe Kolitsch ${ }^{\mathrm{b}}$
}

\begin{abstract}
anstitute for Chemical Technology and Analytics, Division of Structural Chemistry, TU Wien, Getreidemarkt 9/164-SC, 1060 Vienna, Austria, and ${ }^{\mathbf{b}}$ Naturhistorisches Museum Wien, Burgring 7, 1010 Wien, and Institut für Mineralogie und Kristallographie, Universität Wien, Althanstrasse 14, 1090 Wien, Austria. *Correspondence e-mail:

karolina.schwendtner@tuwien.ac.at
\end{abstract}

The crystal structures of hydrothermally synthesized ( $T=493 \mathrm{~K}, 7-9 \mathrm{~d})$ rubidium gallium bis[hydrogenarsenate $(\mathrm{V})], \mathrm{RbGa}\left(\mathrm{HAsO}_{4}\right)_{2}$, and rubidium digallium arsenic $(\mathrm{V})$ hexa[hydrogenarsenate $(\mathrm{V})], \mathrm{RbGa}_{2} \mathrm{As}\left(\mathrm{HAsO}_{4}\right)_{6}$, were solved by single-crystal X-ray diffraction. Both compounds have tetrahedraloctahedral framework topologies. The $M^{+}$cations are located in channels of the respective framework. $\mathrm{RbGa}\left(\mathrm{HAsO}_{4}\right)_{2}$ crystallizes in the $\mathrm{RbFe}\left(\mathrm{HPO}_{4}\right)_{2}$ structure type $(R \overline{3} c)$, while $\mathrm{RbGa}_{2} \mathrm{As}\left(\mathrm{HAsO}_{4}\right)_{6}$ adopts the structure type of $\mathrm{RbAl}_{2} \mathrm{As}\left(\mathrm{HAsO}_{4}\right)_{6}(R \overline{3} c)$, which represents a modification of the $\mathrm{RbFe}\left(\mathrm{HPO}_{4}\right)_{2}$ structure type. In this modification, one third of the $M^{3+} \mathrm{O}_{6}$ octahedra are replaced by $\mathrm{AsO}_{6}$ octahedra, and two thirds of the voids in the structure, which are usually filled by $\mathrm{M}^{+}$cations, remain empty to achieve charge balance.

\section{Chemical context}

Compounds with mixed tetrahedral-octahedral (T-O) framework structures feature a broad range of different atomic arrangements, resulting in topologies with various interesting properties, such as ion exchange (Masquelier et al., 1996) and ion conductivity (Chouchene et al., 2017), as well as unusual piezoelectric (Ren et al., 2015), magnetic (Ouerfelli et al., 2007) or nonlinear optical features (frequency doubling) (Sun et al., 2017). In order to further increase the insufficient knowledge about the crystal chemistry and structure types of arsenates, a comprehensive study of the system $M^{+}-M^{3+}-\mathrm{O}-$ $(\mathrm{H})-\mathrm{As}^{5+}\left(M^{+}=\mathrm{Li}, \mathrm{Na}, \mathrm{K}, \mathrm{Rb}, \mathrm{Cs}, \mathrm{Ag}, \mathrm{Tl}, \mathrm{NH}_{4} ; M^{3+}=\mathrm{Al}, \mathrm{Ga}\right.$, $\mathrm{In}, \mathrm{Sc}, \mathrm{Fe}, \mathrm{Cr}, \mathrm{Tl}$ ) was undertaken, which led to a large number of new compounds, most of which have been published (Schwendtner \& Kolitsch, 2007, 2017, 2018a,b, and references therein).

Among the many different structure types found during our study, one atomic arrangement, i.e. the $\mathrm{RbFe}\left(\mathrm{HPO}_{4}\right)_{2}$ type (Lii $\& \mathrm{Wu}, 1994$; rhombohedral, $R \overline{3} c$ ), was found to show a large crystal-chemical flexibility which allows the incorporation of a wide variety of cations. A total of nine representatives of this structure type are presently known among $M^{+} M^{3+}\left({\mathrm{H} T \mathrm{O}_{4}}_{4}\right)_{2}$ ( $T=\mathrm{P}$, As) compounds containing $\mathrm{Rb}$ or $\mathrm{Cs}$ as the $M^{+}$cation and $\mathrm{Al}, \mathrm{Ga}, \mathrm{Fe}$ or In as the $M^{3+}$ cation (Lesage et al., 2007; Lii \& Wu, 1994; Schwendtner \& Kolitsch, 2017, 2018a,b), including $\mathrm{RbGa}\left(\mathrm{HPO}_{4}\right)_{2}$ (Lesage et al., 2007). One of the title compounds, $\mathrm{RbGa}\left(\mathrm{HAsO}_{4}\right)_{2}$, is another new representative of the $\mathrm{RbFe}\left(\mathrm{HPO}_{4}\right)_{2}$ structure type. The second title compound, $\mathrm{RbGa}_{2} \mathrm{As}\left(\mathrm{HAsO}_{4}\right)_{6}$, is the third representative of a recently described variation of the $\mathrm{RbFe}\left(\mathrm{HPO}_{4}\right)_{2}$ type, the $\mathrm{RbAl}_{2}-$ As $\left(\mathrm{HAsO}_{4}\right)_{6}$ type. It also crystallizes in $R \overline{3} c$ and up to now members with $\mathrm{RbAl}$ and $\mathrm{CsFe}$ as $M^{+} M^{3+}$ cation combinations 
Table 1

Hydrogen-bond geometry $\left(\AA{ }^{\circ}{ }^{\circ}\right)$ for $\mathrm{RbGa}\left(\mathrm{HAsO}_{4}\right)_{2}$.

\begin{tabular}{lllll}
\hline$D-\mathrm{H} \cdots A$ & $D-\mathrm{H}$ & $\mathrm{H} \cdots A$ & $D \cdots A$ & $D-\mathrm{H} \cdots A$ \\
\hline $\mathrm{O} 3-\mathrm{H} \cdots \mathrm{O} 4^{\mathrm{xxi}}$ & $0.85(3)$ & $1.76(3)$ & $2.598(2)$ & $168(4)$ \\
\hline
\end{tabular}

Symmetry code: (xxi) $y, x-1,-z+\frac{3}{2}$.

are known (Schwendtner \& Kolitsch, 2018b). Interestingly, all presently known $M^{+} M^{3+}$ combinations adopting this new structure type also have representatives adopting the $\mathrm{RbFe}\left(\mathrm{HPO}_{4}\right)_{2}$ type. It thus seems likely that more of the known $\mathrm{RbFe}\left(\mathrm{HPO}_{4}\right)_{2}$-type arsenates would also adopt the new $\mathrm{RbAl}_{2} \mathrm{As}\left(\mathrm{HAsO}_{4}\right)_{6}$-type atomic arrangement under formally 'dry' synthesis conditions (see §3). RbGa ${ }_{2} \mathrm{As}$ $\left(\mathrm{HAsO}_{4}\right)_{6}$ is a rare example of a compound containing $\mathrm{AsO}_{6}$ octahedra. Out of all reported arsenates(V), only about $3 \%$ contain $\mathrm{AsO}_{6}$ polyhedra, according to our earlier review paper (Schwendtner \& Kolitsch, 2007), which provides an overview of all known compounds containing $\mathrm{AsO}_{6}$ groups and their bond-length statistics. At present, 37 compounds containing As in an octahedral coordination are known (Schwendtner \& Kolitsch, 2018b); $\mathrm{RbGa}_{2} \mathrm{As}\left(\mathrm{HAsO}_{4}\right)_{6}$ represents the $38^{\text {th }}$ member of this class of compounds. While $12 \mathrm{Rb}$ - and Gacontaining phosphates are contained in the ICSD (FIZ, 2018),

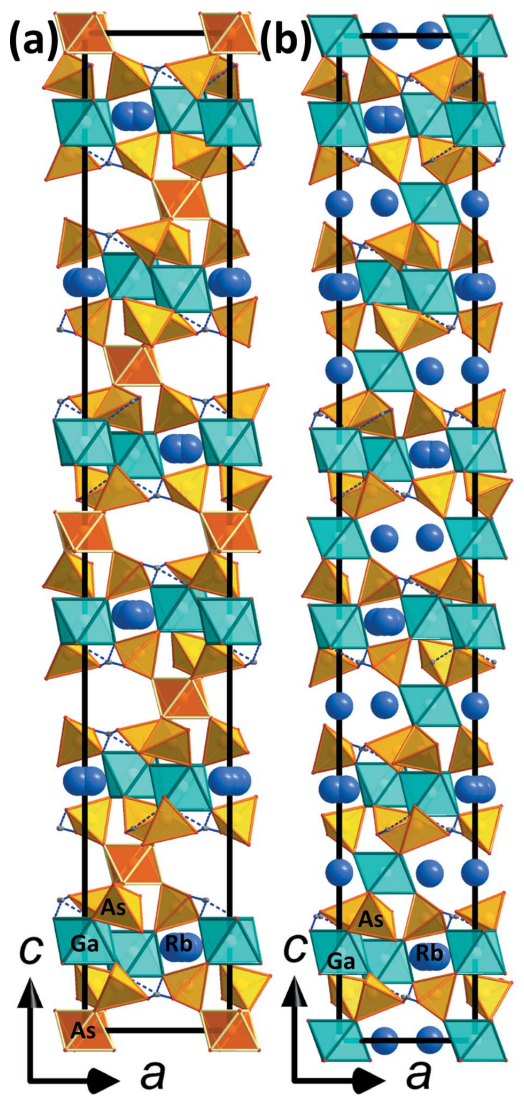

Figure 1

Crystal structure drawings of (a) $\mathrm{RbGa}_{2} \mathrm{As}\left(\mathrm{HAsO}_{4}\right)_{6}$ and (b) RbGa$\left(\mathrm{HAsO}_{4}\right)_{2}$ in views along the $b$ axis. A part of the $\mathrm{GaO}_{6}$ octahedra is replaced by $\mathrm{AsO}_{6}$ octahedra in $\mathrm{RbGa}_{2} \mathrm{As}\left(\mathrm{HAsO}_{4}\right)_{6}$; the corresponding layers (see Figs. 2 and 3 ) are compressed along $c$ and the corresponding void remains vacant of $\mathrm{Rb}$ atoms.
Table 2

Hydrogen-bond geometry $\left(\AA{ }^{\circ}\right)$ for $\mathrm{RbGa}_{2} \mathrm{As}\left(\mathrm{HAsO}_{4}\right)_{6}$.

\begin{tabular}{lllll}
\hline$D-\mathrm{H} \cdots A$ & $D-\mathrm{H}$ & $\mathrm{H} \cdots A$ & $D \cdots A$ & $D-\mathrm{H} \cdots A$ \\
\hline $\mathrm{O} 3-\mathrm{H} \cdots \mathrm{O} 4^{\text {xiv }}$ & $0.80(3)$ & $1.98(3)$ & $2.7314(17)$ & $158(3)$ \\
\hline
\end{tabular}

Symmetry code: (xiv) $y, x-1,-z+\frac{3}{2}$.

only one $\mathrm{Rb}-\mathrm{Ga}$ arsenate, i.e. $\mathrm{RbGaF}_{3}\left(\mathrm{H}_{2} \mathrm{AsO}_{4}\right)$ (Marshall et $a l ., 2015)$, is known so far. Since submitting this paper, another paper dealing with isotypic $M^{+} M_{2}^{3+} \mathrm{As}\left(\mathrm{HAsO}_{4}\right)_{6}$ compounds $\left(M^{+} M^{3+}=\mathrm{TlGa}, \mathrm{CsGa}, \mathrm{CsAl}\right)$ has been published (Schwendtner \& Kolitsch, 2018c).

\section{Structural commentary}

The two title compounds are very closely related to each other and are modifications of a basic tetrahedral-octahedral framework structure featuring interpenetrating channels, which host the $M^{+}$cations (Fig. 1). The two structure types, first reported for $\mathrm{RbFe}\left(\mathrm{HPO}_{4}\right)_{2}(R \overline{3} c$; Lii \& Wu, 1994) and $\mathrm{RbAl}_{2} \mathrm{As}\left(\mathrm{HAsO}_{4}\right)_{6}(R \overline{3} c$; Schwendtner \& Kolitsch, 2018b), are

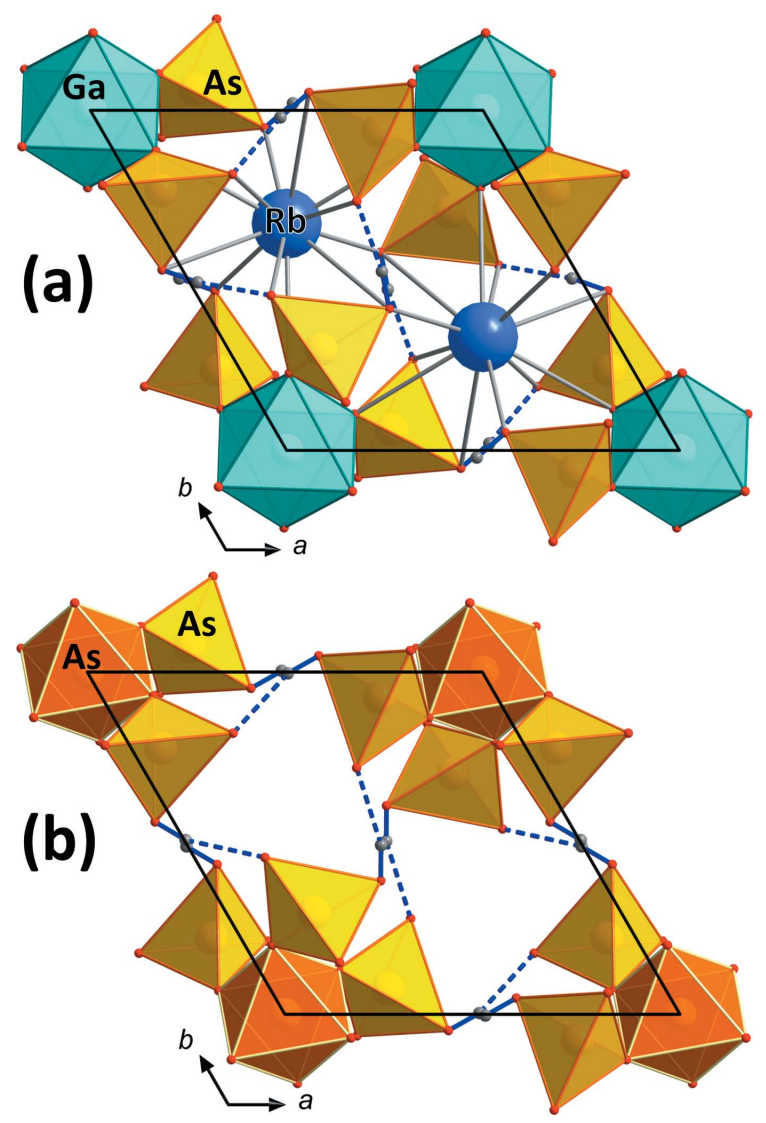

Figure 2

Crystal structure drawings of (a) $\mathrm{RbGa}\left(\mathrm{HAsO}_{4}\right)_{2}$ and (b) $\mathrm{RbGa}_{2} \mathrm{As}$ $\left(\mathrm{HAsO}_{4}\right)_{6}$ inequal layers, viewed along the $c$ axis. In this layer, the $\mathrm{GaO}_{6}$ octahedra are replaced by $\mathrm{AsO}_{6}$ octahedra in $\mathrm{RbGa}_{2} \mathrm{As}\left(\mathrm{HAsO}_{4}\right)_{6}(b)$. Since the unit-cell dimensions in directions $a$ and $b$ are slightly longer in $\mathrm{RbGa}_{2} \mathrm{As}\left(\mathrm{HAsO}_{4}\right)_{6}$ and the $\mathrm{AsO}_{6}$ octahedra are smaller than the corresponding $\mathrm{GaO}_{6}$ octahedra, the $(\mathrm{Ga} / \mathrm{As}) \mathrm{As}_{6} \mathrm{O}_{24}$ units within this layer move further apart - leading to longer $D-\mathrm{H} \cdots A$ distances and a compressed (along $c$ ) void that is too small for $\mathrm{Rb}$ atoms (compare Fig. 1). 


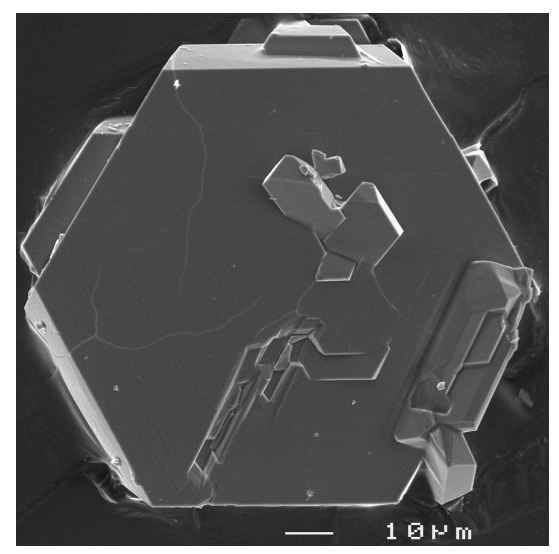

Figure 3

SEM micrograph of the pseudohexagonal tabular crystals of RbGa$\left(\mathrm{HAsO}_{4}\right)_{2}$.

also related to the triclinic $\left(\mathrm{NH}_{4}\right) \mathrm{Fe}\left(\mathrm{HPO}_{4}\right)_{2}$ type $(P \overline{1}$; Yakubovich, 1993) and the $\mathrm{RbAl}\left(\mathrm{HAsO}_{4}\right)_{2}$ type (R32; Schwendtner $\&$ Kolitsch, 2018b). The fundamental building unit in all these structure types contains $M^{3+} \mathrm{O}_{6}$ octahedra which are connected via their six corners to six protonated $\mathrm{AsO}_{4}$ tetrahedra, thereby forming an $M^{3+} \mathrm{As}_{6} \mathrm{O}_{24}$ unit. These units are in turn connected via three corners to other $M^{3+} \mathrm{O}_{6}$ octahedra. The free protonated corner of each $\mathrm{AsO}_{4}$ tetrahedron forms a hydrogen bond to the neighbouring $M^{3+} \mathrm{As}_{6} \mathrm{O}_{24}$ group (Fig. 2). The $M^{3+} \mathrm{As}_{6} \mathrm{O}_{24}$ units are arranged in layers perpendicular to the $c_{\text {hex }}$ axis (Fig. 1). The units within these layers are held together by medium-strong hydrogen bonds (Tables 1 and 2). Both title compounds invariably show a very similar crystal habit: strongly pseudohexagonal to pseudo-octahedral ( $c f$. Fig. 3).

The new compound $\mathrm{RbGa}_{2} \mathrm{As}\left(\mathrm{HAsO}_{4}\right)_{6}$ could only be grown by 'dry' hydrothermal techniques (without the addition of water). The extreme abundance of As during the synthesis and the formation of a melt of arsenic acid promotes the formation of this novel structure type and endorses the octahedral coordination of As. The substitution of one third of all $\mathrm{Ga}^{3+}$ cations by $\mathrm{As}^{5+}$ requires that two thirds of all $\mathrm{Rb}^{+}$cations are omitted to achieve charge balance (compare Figs. $1 a, 1 b$, $2 a$ and $2 b$ ). This substitution also has an effect on the unit-cell parameters (Table 3) and the pore diameter. Since $\mathrm{GaO}_{6}$ is only replaced by $\mathrm{AsO}_{6}$ in every second layer (perpendicular to the $c$ axis), the $a$ axis must remain long enough to still be able to house the $\mathrm{GaO}_{6}$ in the layers between. The effect of the smaller $\mathrm{AsO}_{6}$ octahedra is therefore mainly reflected by a strong compression of about $5 \%$ along the $c$ axis, while the $a$ axis becomes even slightly longer compared to $\mathrm{RbGa}$ $\left(\mathrm{HAsO}_{4}\right)_{2}$. Due to the comparatively smaller $\mathrm{AsO}_{6}$ octahedra, the $(\mathrm{Ga} / \mathrm{As}) \mathrm{As}_{6} \mathrm{O}_{24}$ units are further apart in $\mathrm{RbGa}_{2} \mathrm{As}$ $\left(\mathrm{HAsO}_{4}\right)_{6}$ and the encased void is compressed along $c$, making it too small to house $\mathrm{Rb}^{+}$cations (Figs. 1 and 2). This effect is also reflected by the considerably elongated hydrogen bond in $\mathrm{RbGa}_{2} \mathrm{As}\left(\mathrm{HAsO}_{4}\right)_{6}$. While these bonds, which connect neighbouring $(\mathrm{Ga} / \mathrm{As}) \mathrm{As}_{6} \mathrm{O}_{24}$ groups, are very strong in $\mathrm{RbGa}\left(\mathrm{HAsO}_{4}\right)_{2}[D-\mathrm{H} \cdots A=2.598(2) \AA]$, they are much longer in $\mathrm{RbGa}_{2} \mathrm{As}\left(\mathrm{HAsO}_{4}\right)_{6}$ [2.7314 (17) $\AA$; compare Tables 1 and 2]. The second layer, in contrast, remains practically identical in both compounds and contains $\mathrm{Rb}$ atoms with a slight positional disorder (Fig. 4). In both compounds, the Rb atoms are 12-coordinated (Figs. 2 and 3), and the average $\mathrm{Rb}-\mathrm{O}$ bond lengths in $\mathrm{RbGa}_{2} \mathrm{As}\left(\mathrm{HAsO}_{4}\right)_{6}(3.433 \AA)$ are longer than the longest average bond length in $\mathrm{RbO}_{12}$ polyhedra of $3.410 \AA$ A reported so far (Gagné \& Hawthorne, 2016), thus leading to rather low bond-valence sums (BVSs; Gagné \& Hawthorne, 2015) of only 0.59 valence units (v.u.), whereas the corresponding BVSs are 0.82 and 0.84 v.u. for $\mathrm{RbGa}\left(\mathrm{HAsO}_{4}\right)_{2}$. These loose bondings lead to considerable positional disorder of the $\mathrm{Rb}^{+}$cations in these voids, which were modelled with two $\mathrm{Rb}$ positions, between 0.41 (2) and 0.42 (4) A apart. While position $\mathrm{Rb} 1 A$ in the centre of the large framework void in $\mathrm{RbGa}_{2} \mathrm{As}\left(\mathrm{HAsO}_{4}\right)_{6}$ has only $77 \%$ occupancy compared to the off-centre position $\mathrm{Rb} 1 B$ (with occupancy $23 \%$ ), in RbGa$\left(\mathrm{HAsO}_{4}\right)_{2}$, the central position $\mathrm{Rb} 1 A$ has $91 \%$ occupancy. Similar behaviour was observed for the isotypic CsFe and RbAl compounds (Schwendtner \& Kolitsch, 2018b), as well as isotypic phosphates (Lesage et al., 2007).

A further indirect effect of the substituting $\mathrm{AsO}_{6}$ octahedra is a distinct change in the $\mathrm{As}-\mathrm{O}$ distances of the $\mathrm{AsO}_{4}$ tetrahedra. The average As-O distance in the protonated $\mathrm{AsO}_{4}$ tetrahedra, with values between 1.688 and $1.689 \AA$, is in
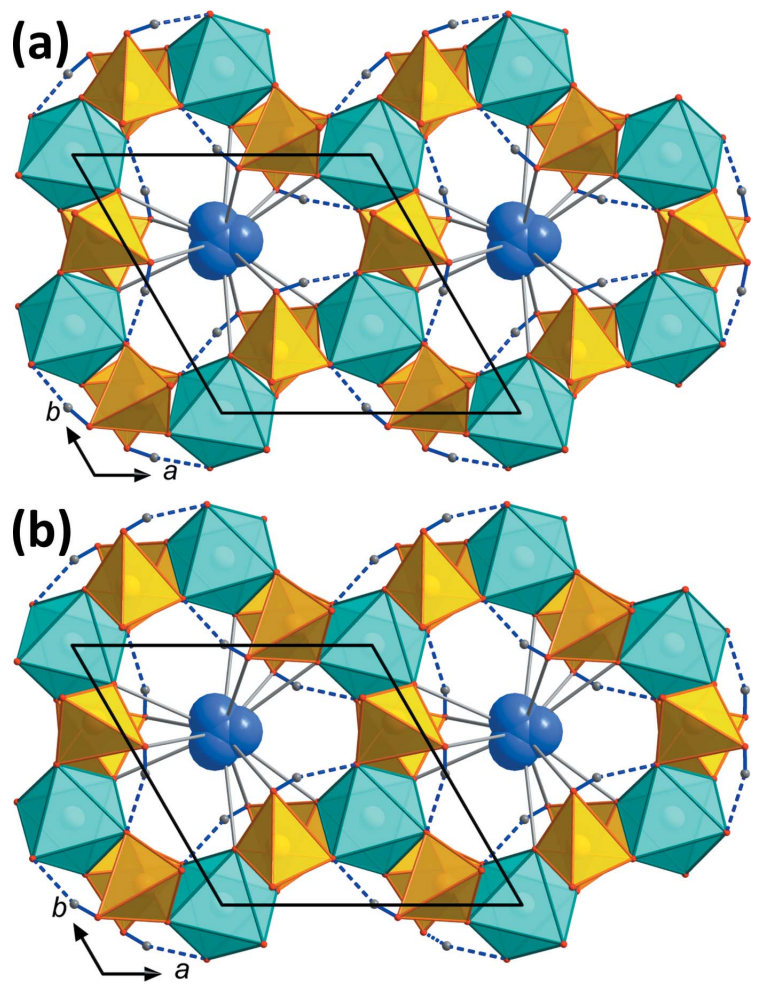

Figure 4

Crystal structure drawing of (a) $\mathrm{RbGa}\left(\mathrm{HAsO}_{4}\right)_{2}$ and (b) $\mathrm{RbGa}_{2}$ $\mathrm{As}\left(\mathrm{HAsO}_{4}\right)_{6}$ equal layers, viewed along the $a$ axis. In these topologically equivalent layers, there are no visible differences between the two structure types apart from very minor changes in the hydrogen-bond geometries. The $\mathrm{Rb}$ atoms in both compounds show a slight positional disorder and are 12-coordinated. 
Table 3

Experimental details.

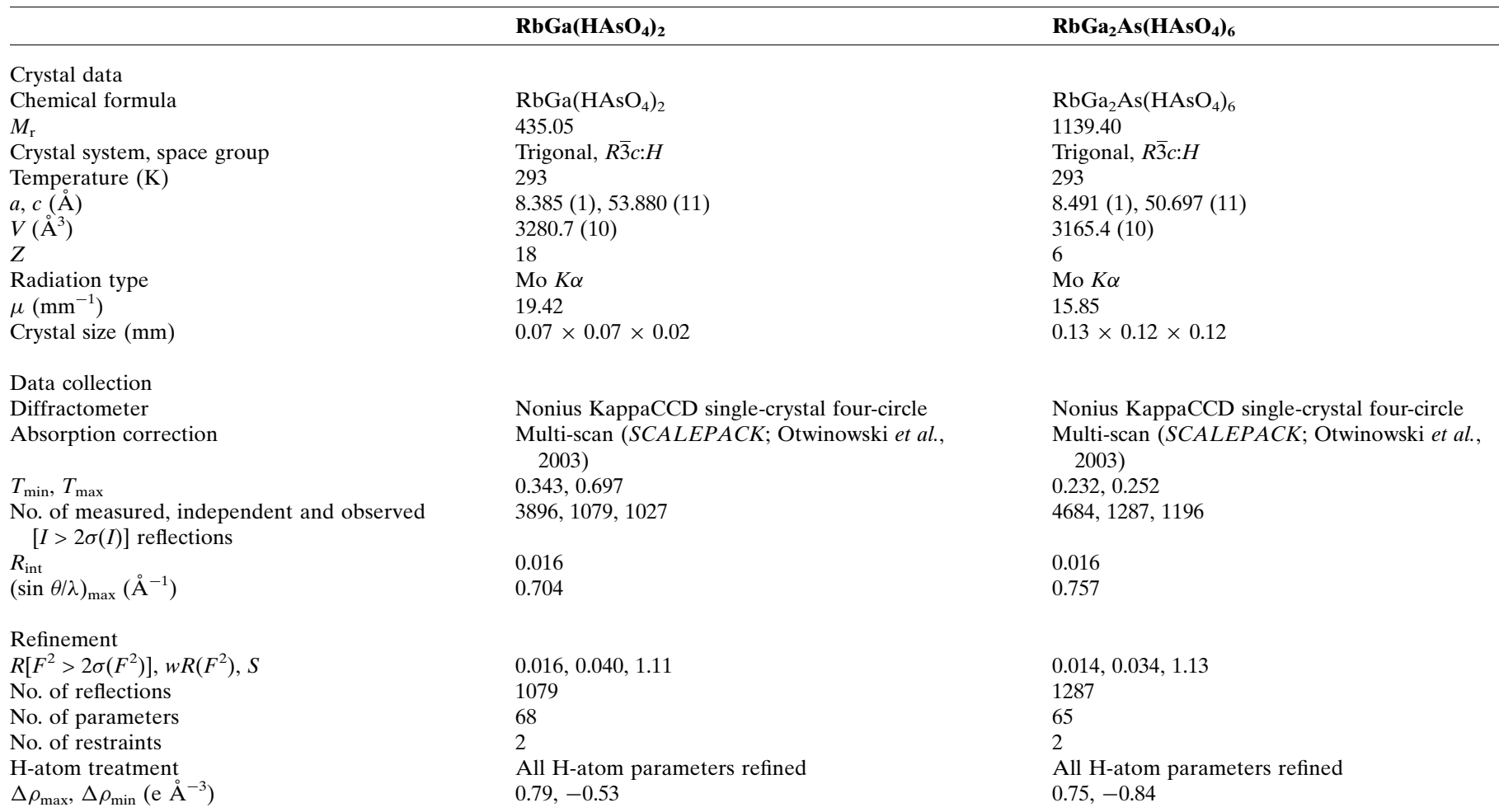

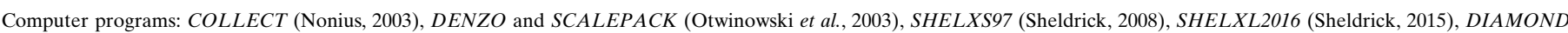
(Brandenburg, 2005) and publCIF (Westrip, 2010).

both compounds very close to the statistical average of 1.686 (10) ^ (Schwendtner, 2008). Also the BVSs (Gagné \& Hawthorne, 2015) are close to ideal values (4.98-5.00 v.u.). In $\mathrm{RbGa}\left(\mathrm{HAsO}_{4}\right)_{2}$, the $\mathrm{HAsO}_{4}$ tetrahedra show a typical distortion, with three short As-O distances to attached $\mathrm{GaO}_{6}$ octahedra and one elongated As-O bond length for the

Table 4

Selected bond lengths $(\AA)$ for $\mathrm{RbGa}\left(\mathrm{HAsO}_{4}\right)_{2}$.

\begin{tabular}{llll}
\hline $\mathrm{Rb} 1 A-\mathrm{O} 3^{\mathrm{i}}$ & $3.197(2)$ & $\mathrm{Rb} 2-\mathrm{O} 4^{\mathrm{xi}}$ & $3.4960(16)$ \\
$\mathrm{Rb} 1 A-\mathrm{O} 3^{\mathrm{ii}}$ & $3.197(2)$ & $\mathrm{Rb} 2-3^{\mathrm{xii}}$ & $3.5327(19)$ \\
$\mathrm{Rb} 1 A-\mathrm{O} 3^{\mathrm{iii}}$ & $3.197(2)$ & $\mathrm{Rb} 2-\mathrm{O} 3^{\mathrm{xii}}$ & $3.533(2)$ \\
$\mathrm{Rb} 1 A-\mathrm{O} 3^{\mathrm{iv}}$ & $3.197(2)$ & $\mathrm{Rb} 2-\mathrm{O} 3^{\mathrm{xiv}}$ & $3.5328(19)$ \\
$\mathrm{Rb} 1 A-\mathrm{O} 3^{\mathrm{v}}$ & $3.197(2)$ & $\mathrm{Ga} 1-\mathrm{O} 2^{\mathrm{xv}}$ & $1.9596(14)$ \\
$\mathrm{Rb} 1 A-\mathrm{O} 3$ & $3.197(2)$ & $\mathrm{Ga} 1-\mathrm{O} 2^{\mathrm{iii}}$ & $1.9597(15)$ \\
$\mathrm{Rb} 1 A-\mathrm{O} 2^{\mathrm{ii}}$ & $3.3698(16)$ & $\mathrm{Ga} 1-\mathrm{O} 2^{\mathrm{xvi}}$ & $1.9597(15)$ \\
$\mathrm{Rb} 1 A-\mathrm{O} 2^{\mathrm{iii}}$ & $3.3698(16)$ & $\mathrm{Ga} 1-\mathrm{O} 4^{\mathrm{xvii}}$ & $1.9690(15)$ \\
$\mathrm{Rb} 1 A-\mathrm{O} 2^{\mathrm{v}}$ & $3.3699(16)$ & $\mathrm{Ga} 1-\mathrm{O} 4^{\mathrm{iv}}$ & $1.9690(15)$ \\
$\mathrm{Rb} 1 A-\mathrm{O} 2$ & $3.3699(16)$ & $\mathrm{Ga} 1-\mathrm{O} 4^{\mathrm{xvii}}$ & $1.9690(15)$ \\
$\mathrm{Rb} 1 A-\mathrm{O} 2^{\mathrm{i}}$ & $3.3699(15)$ & $\mathrm{Ga} 2-\mathrm{O} 1^{\mathrm{vii}}$ & $1.9625(15)$ \\
$\mathrm{Rb} 1 A-\mathrm{O} 2^{\mathrm{iv}}$ & $3.3699(15)$ & $\mathrm{Ga} 2-\mathrm{O} 1^{\mathrm{xiv}}$ & $1.9625(16)$ \\
$\mathrm{Rb} 2-\mathrm{O} 3$ & $2.9346(17)$ & $\mathrm{Ga} 2-\mathrm{O} 1^{\mathrm{xix}}$ & $1.9625(15)$ \\
$\mathrm{Rb} 2-\mathrm{O} 3^{\mathrm{iv}}$ & $2.9347(17)$ & $\mathrm{Ga} 2-\mathrm{O} 1^{\mathrm{iv}}$ & $1.9626(15)$ \\
$\mathrm{Rb} 2-\mathrm{O} 3^{\mathrm{iii}}$ & $2.9347(17)$ & $\mathrm{Ga} 2-\mathrm{O} 1^{\mathrm{xvii}}$ & $1.9626(16)$ \\
$\mathrm{Rb} 2-\mathrm{O} 1^{\mathrm{vi}}$ & $3.3714(16)$ & $\mathrm{Ga} 2-\mathrm{O} 1^{\mathrm{xvii}}$ & $1.9626(15)$ \\
$\mathrm{Rb} 2-\mathrm{O} 1^{\mathrm{vii}}$ & $3.3715(16)$ & $\mathrm{As}-\mathrm{O} 1^{\mathrm{xx}}$ & $1.6576(15)$ \\
$\mathrm{Rb} 2-\mathrm{O} 1^{\mathrm{viii}}$ & $3.3715(17)$ & $\mathrm{As}-\mathrm{O} 2$ & $1.6724(15)$ \\
$\mathrm{Rb} 2-\mathrm{O} 4^{\mathrm{ix}}$ & $3.4960(16)$ & $\mathrm{As}-\mathrm{O} 4^{\mathrm{ii}}$ & $1.6805(15)$ \\
$\mathrm{Rb} 2-\mathrm{O} 4^{\mathrm{x}}$ & $3.4960(17)$ & $\mathrm{As}-\mathrm{O} 3$ & $1.7417(17)$ \\
\hline
\end{tabular}

Symmetry codes: (i) $x-y,-y,-z+\frac{3}{2}$; (ii) $-x,-x+y,-z+\frac{3}{2}$; (iii) $-x+y,-x, z$; (iv) $-y, x-y, z$; (v) $y, x,-z+\frac{3}{2}$; (vi) $-x+\frac{2}{3},-y-\frac{2}{3},-z+\frac{4}{3}$; (vii) $x-y-\frac{4}{3}, x-\frac{2}{3},-z+\frac{4}{3}$; protonated $\mathrm{O}$ atom involved in the $\mathrm{O}-\mathrm{H}$ bond. That bond length (Table 4) in $\mathrm{RbGa}\left(\mathrm{HAsO}_{4}\right)_{2}$ is slightly longer $[1.7417(17) \AA]$ than the average distance of $\mathrm{As}-\mathrm{O} \cdots \mathrm{H}$ bonds in $\mathrm{HAsO}_{4}$ groups [1.72 (3) $\AA$; Schwendtner, 2008]. In contrast, $\mathrm{RbGa}_{2} \mathrm{As}\left(\mathrm{HAsO}_{4}\right)_{6}$ has two short ${ }^{[4]} \mathrm{As}-\mathrm{O}$ bond lengths to neighbouring $\mathrm{GaO}_{6}$ octahedra, but the ${ }^{[4]} \mathrm{As}-\mathrm{O}$ bond length of the $\mathrm{O}$ atom shared with the $\mathrm{AsO}_{6}$ octahedra is also elongated $[1.7100(11) \AA]$ due to ${ }^{[4]} \mathrm{As}-\mathrm{O}-{ }^{[6]} \mathrm{As}$ repulsion. The ${ }^{[4]} \mathrm{As}-\mathrm{OH}$ bond is therefore shortened to 1.7122 (13) $\AA$ (Table 5). The average As-O distances in the $\mathrm{AsO}_{6}$ octahedra are the shortest average distances of $\mathrm{AsO}_{6}$ octahedra found so far, i.e. $1.807 \AA$, leading to rather high BVSs of 5.33 v.u. (after Gagné \& Hawthorne, 2015). The grand mean As- $\mathrm{O}$ bond distance in $\mathrm{AsO}_{6}$ octahedra in inorganic compounds is 1.830 (2) $\AA$ according to Schwendtner \& Kolitsch (2007a); this value was determined on $33 \mathrm{AsO}_{6}$ octahedra of 31 compounds. Gagné \& Hawthorne (2018) determined an identical, but less precise, value of 1.830 (28) $\AA$, based on only $13 \mathrm{AsO}_{6}$ octahedra in $\mathrm{AsO}_{6}$ containing compounds meeting all selection criteria as defined in Gagné \& Hawthorne (2016). However, a larger number of compounds meeting these criteria were not used by Gagné \& Hawthorne (2018) for unknown reasons. The average $\mathrm{Ga}-\mathrm{O}$ bond lengths of the octahedrally coordinated Ga cations (1.962-1.964 ̊) are slightly shorter than the grand mean average of 1.978 (17) $~$ (Gagné \& Hawthorne, 2018), explaining the corresponding BVSs of 3.10 to 3.11 v.u. 
Table 5

Selected bond lengths $(\AA)$ for $\mathrm{RbGa}_{2} \mathrm{As}\left(\mathrm{HAsO}_{4}\right)_{6}$.

\begin{tabular}{llll}
\hline $\mathrm{Rb} 1 A-\mathrm{O}^{\mathrm{i}}$ & $3.4212(16)$ & $\mathrm{Ga} 1-\mathrm{O} 4^{\mathrm{vii}}$ & $1.9623(11)$ \\
$\mathrm{Rb} 1 A-\mathrm{O}^{\mathrm{ii}}$ & $3.4212(16)$ & $\mathrm{Ga} 1-\mathrm{O} 2^{\mathrm{viii}}$ & $1.9625(11)$ \\
$\mathrm{Rb} 1 A-\mathrm{O}^{\mathrm{iii}}$ & $3.4212(15)$ & $\mathrm{Ga} 1-\mathrm{O} 2^{\mathrm{iii}}$ & $1.9625(11)$ \\
$\mathrm{Rb} 1 A-\mathrm{O}^{\mathrm{iv}}$ & $3.4212(16)$ & $\mathrm{Ga} 1-\mathrm{O} 2^{\mathrm{ix}}$ & $1.9625(11)$ \\
$\mathrm{Rb} 1 A-\mathrm{O}^{\mathrm{v}}$ & $3.4212(15)$ & $\mathrm{As} 1-\mathrm{O} 1^{\mathrm{x}}$ & $1.8067(11)$ \\
$\mathrm{Rb} 1 A-\mathrm{O} 3$ & $3.4213(16)$ & $\mathrm{As} 1-\mathrm{O} 1^{\mathrm{xi}}$ & $1.8068(11)$ \\
$\mathrm{Rb} 1 A-\mathrm{O} 2^{\mathrm{ii}}$ & $3.4438(12)$ & $\mathrm{As} 1-\mathrm{O} 1^{\mathrm{xii}}$ & $1.8068(11)$ \\
$\mathrm{Rb} 1 A-\mathrm{O} 2^{\mathrm{iii}}$ & $3.4438(12)$ & $\mathrm{As} 1-\mathrm{O} 1^{\mathrm{v}}$ & $1.8068(11)$ \\
$\mathrm{Rb} 1 A-\mathrm{O} 2$ & $3.4438(12)$ & $\mathrm{As} 1-\mathrm{O} 1^{\mathrm{vii}}$ & $1.8068(11)$ \\
$\mathrm{Rb} 1 A-\mathrm{O} 2^{\mathrm{iv}}$ & $3.4438(12)$ & $\mathrm{As} 1-\mathrm{O} 1^{\mathrm{vi}}$ & $1.8068(11)$ \\
$\mathrm{Rb} 1 A-\mathrm{O} 2^{\mathrm{i}}$ & $3.4438(12)$ & $\mathrm{As} 2-\mathrm{O} 2$ & $1.6658(11)$ \\
$\mathrm{Rb} 1 A-\mathrm{O} 2^{\mathrm{v}}$ & $3.4438(12)$ & $\mathrm{As} 2-\mathrm{O} 4^{\mathrm{ii}}$ & $1.6670(11)$ \\
$\mathrm{Ga} 1-\mathrm{O} 4^{\mathrm{vi}}$ & $1.9623(12)$ & $\mathrm{As} 2-\mathrm{O} 1^{\mathrm{vii}}$ & $1.7100(11)$ \\
$\mathrm{Ga} 1-\mathrm{O} 4^{\mathrm{v}}$ & $1.9623(11)$ & $\mathrm{As} 2-\mathrm{O} 3$ & $1.7122(13)$ \\
\hline
\end{tabular}

Symmetry codes: (i) $x-y,-y,-z+\frac{3}{2}$; (ii) $-x,-x+y,-z+\frac{3}{2}$; (iii) $-x+y,-x, z$; (iv) $y, x,-z+\frac{3}{2}$; (v) $-y, x-y, z$; (vi) $x, y+1, z$; (vii) $-x+y+1,-x+1, z$; (viii) $-y, x-y+1, z ; \quad$ (ix) $\quad x+1, y+1, z ; \quad$ (x) $\quad x-y-\frac{1}{3}, x+\frac{1}{3},-z+\frac{4}{3} ; \quad$ (xi) $y+\frac{2}{3},-x+y+\frac{4}{3},-z+\frac{4}{3}$; (xii) $-x+\frac{2}{3},-y+\frac{1}{3},-z+\frac{4}{3}$; (xiii) $x-1, y, z$.

\section{Synthesis and crystallization}

The compounds were grown by hydrothermal synthesis at 493 K (7 d, autogeneous pressure, slow furnace cooling) using Teflon-lined stainless steel autoclaves with an approximate filling volume of $2 \mathrm{ml}$. Reagent-grade $\mathrm{Rb}_{2} \mathrm{CO}_{3}, \mathrm{Ga}_{2} \mathrm{O}_{3}$ and $\mathrm{H}_{3} \mathrm{AsO}_{4} \cdot 0.5 \mathrm{H}_{2} \mathrm{O}$ were used as starting reagents in approximate volume ratios of Rb:Ga:As of 1:1:3 for both synthesis batches. For $\mathrm{RbGa}\left(\mathrm{HAsO}_{4}\right)_{2}$ the vessels were filled with distilled water to about $70 \%$ of their inner volumes, which led to initial and final $\mathrm{pH}$ values of 1.5. The reaction product was washed thoroughly with distilled water, filtered and dried at room temperature. $\mathrm{RbGa}\left(\mathrm{HAsO}_{4}\right)_{2}$ formed colourless pseudohexagonal platelets (Fig. 3) and is stable in air.

For $\mathrm{RbGa}_{2} \mathrm{As}\left(\mathrm{HAsO}_{4}\right)_{6}$, which contains As in both tetrahedral and octahedral coordination, no additional water was added and arsenic acid was present in excess to promote the growth of crystals from a melt or even vapour of arsenic acid under extremely acidic conditions. $\mathrm{RbGa}_{2} \mathrm{As}\left(\mathrm{HAsO}_{4}\right)_{6}$ formed large colourless pseudo-octahedral crystals accompanied by small colourless twinned crystals of $\mathrm{RbH}_{3} \mathrm{As}_{4} \mathrm{O}_{12}$ (Schwendtner \& Kolitsch, 2007). The crystals of $\mathrm{RbGa}_{2} \mathrm{As}-$ $\left(\mathrm{HAsO}_{4}\right)_{6}$ were extracted mechanically and not further washed; they are hygroscopic and decompose slowly over a period of several years to an amorphous gel and a new, strongly protonated diarsenate containing $\mathrm{Rb}$ and $\mathrm{Ga}(P 321$, publication in preparation). This slow partial alteration is illustrated in an X-ray powder diffraction pattern (Fig. 5).

A measured X-ray powder diffraction diagram of $\mathrm{RbGa}-$ $\left(\mathrm{HAsO}_{4}\right)_{2}$ was deposited at the International Centre for Diffraction Data under PDF number 00-057-0239 (Wohlschlaeger et al., 2006).

\section{Experimental and refinement}

Crystal data, data collection and structure refinement are given in Table 3. For the refinement of $\mathrm{RbGa}\left(\mathrm{HAsO}_{4}\right)_{2}$, the coordinates of $\mathrm{RbFe}\left(\mathrm{HPO}_{4}\right)_{2}$ ( $\left.\mathrm{Lii} \& \mathrm{Wu}, 1994\right)$ were used for the final refinement steps. $\mathrm{H}$ atoms were then located from difference Fourier maps and added to the model. For the refinement of $\mathrm{RbGa}_{2} \mathrm{As}\left(\mathrm{HAsO}_{4}\right)_{6}$, the model for $\mathrm{RbAl}_{2}$ $\mathrm{As}\left(\mathrm{HAsO}_{4}\right)_{6}($ Schwendtner \& Kolitsch, 2018b) was used as a starting point. In both compounds, $\mathrm{O}-\mathrm{H}$ bonds were restrained to $0.9 \pm 0.04 \AA$. During the last refinement steps, residual electron-density peaks of up to 3.83 and $1.16 \mathrm{e}^{-3}$ were located 0.63 and $0.68 \AA$ from the $\mathrm{Rb}$ sites in $\mathrm{RbGa}_{2}$ $\mathrm{As}\left(\mathrm{HAsO}_{4}\right)_{6}$ and $\mathrm{RbGa}\left(\mathrm{HAsO}_{4}\right)_{2}$, respectively, suggesting irregular displacement parameters and split positions, similar to what was found for $\mathrm{RbFe}\left(\mathrm{HPO}_{4}\right)_{2}$-type $\mathrm{RbAl}\left(\mathrm{HPO}_{4}\right)_{2}$ (Lesage et al., 2007). Therefore, a further position, Rb1B, was included in both refinements, which refined to low occupancies and led to considerable decreases in the $R$ factors and weight parameters for both compounds. The bulk occupancies of $\mathrm{Rb} 1 A+\mathrm{Rb} 1 B$ were constrained to give a total occupancy of 1.00. The final residual electron densities in both compounds are $<1$ e $\AA^{-3}$.

\section{Acknowledgements}

The authors acknowledge the TU Wien University Library for financial support through its Open Access Funding Program.

\section{Funding information}

Funding for this research was provided by: Doc fForte Fellowship of the Austrian Academy of Sciences to K. Schwendtner.

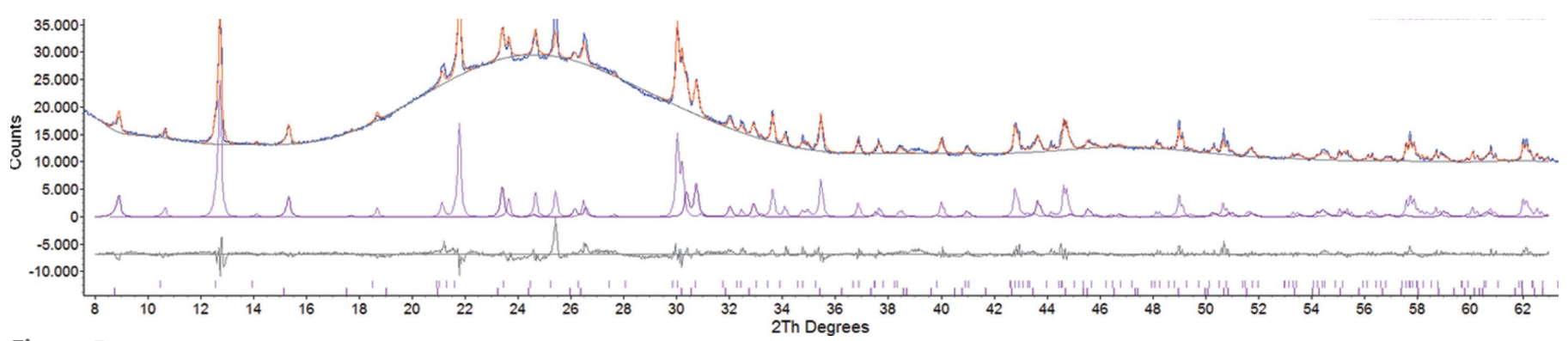

Figure 5

Graph of the Rietveld refinement (TOPAS; Bruker, 2009) of $\mathrm{RbGa}_{2} \mathrm{As}\left(\mathrm{HAsO}_{4}\right)_{6}$, showing the partial alteration of the pseudo-octahedral crystals after an 11-year storage in air. The crystals were hygroscopic and had partly transformed to an amorphous mass. The presence of the relics of the unaltered primary crystals are still visible (pink curve), but a newly crystallized overgrowth of extremely fine fibrous crystals could be attributed to a new strongly protonated $\mathrm{Rb}-\mathrm{Ga}$ diarsenate with space group P321 (dark-red curve), which will be the subject of a future publication. 


\section{References}

Brandenburg, K. (2005). DIAMOND. Crystal Impact GbR, Bonn, Germany.

Bruker (2009). TOPAS. Bruker AXS, Karlsruhe, Germany.

Chouchene, S., Jaouadi, K., Mhiri, T. \& Zouari, N. (2017). Solid State Ionics, 301, 78-85.

FIZ (2018). Inorganic Crystal Structure Database. Version build 20180504-0745, 2018.1. Fachinformationszentrum Karlsruhe, 76344 Eggenstein-Leopoldshafen, Germany.

Gagné, O. C. \& Hawthorne, F. C. (2015). Acta Cryst. B71, 562-578.

Gagné, O. C. \& Hawthorne, F. C. (2016). Acta Cryst. B72, 602-625.

Gagné, O. C. \& Hawthorne, F. C. (2018). Acta Cryst. B74, 63-78.

Lesage, J., Adam, L., Guesdon, A. \& Raveau, B. (2007). J. Solid State Chem. 180, 1799-1808.

Lii, K.-H. \& Wu, L.-S. (1994). J. Chem. Soc. A, 10, 1577-1580.

Marshall, K. L., Armstrong, J. A. \& Weller, M. T. (2015). Dalton Trans. 44, 12804-12811.

Masquelier, C., Padhi, A. K., Nanjundaswamy, K. S., Okada, S. \& Goodenough, J. B. (1996). Proceedings of the 37th Power Sources Conference, June 17-20, 1996, pp. 188-191. Cherry Hill, New Jersey. Fort Monmouth, NJ: US Army Research Laboratory.
Nonius (2003). COLLECT. Nonius BV, Delft, The Netherlands. Otwinowski, Z., Borek, D., Majewski, W. \& Minor, W. (2003). Acta Cryst. A59, 228-234.

Ouerfelli, N., Guesmi, A., Molinie, P., Mazza, D., Madani, A., Zid, M. F. \& Driss, A. (2007). J. Solid State Chem. 180, 2942-2949.

Ren, J., Ma, Z., He, C., Sa, R., Li, Q. \& Wu, K. (2015). Comput. Mater. Sci. 106, 1-4.

Schwendtner, K. (2008). PhD thesis, Universität Wien, Austria.

Schwendtner, K. \& Kolitsch, U. (2007). Acta Cryst. B63, 205-215.

Schwendtner, K. \& Kolitsch, U. (2017). Acta Cryst. E73, 15801586.

Schwendtner, K. \& Kolitsch, U. (2018a). Acta Cryst. E74, 766-771.

Schwendtner, K. \& Kolitsch, U. (2018b). Acta Cryst. C74, 721727.

Schwendtner, K. \& Kolitsch, U. (2018c). Acta Cryst. E74, 11631167.

Sheldrick, G. M. (2008). Acta Cryst. A64, 112-122.

Sheldrick, G. M. (2015). Acta Cryst. C71, 3-8.

Sun, Y., Yang, Z., Hou, D. \& Pan, S. (2017). RSC Adv. 7, 2804-2809.

Westrip, S. P. (2010). J. Appl. Cryst. 43, 920-925.

Wohlschlaeger, A., Lengauer, C. \& Tillmanns, E. (2006). ICDD Grant-in-Aid. University of Vienna, Austria.

Yakubovich, O. V. (1993). Kristallografiya, 38, 43-48. 


\section{supporting information}

Acta Cryst. (2018). E74, 1244-1249 [https://doi.org/10.1107/S2056989018011180]

Two new Rb-Ga arsenates: $\mathrm{RbGa}\left(\mathrm{HAsO}_{4}\right)_{2}$ and $\mathrm{RbGa} 2 \mathrm{As}\left(\mathrm{HAsO}_{4}\right)_{6}$

\section{Karolina Schwendtner and Uwe Kolitsch}

\section{Computing details}

For both structures, data collection: COLLECT (Nonius, 2003); cell refinement: SCALEPACK (Otwinowski et al., 2003); data reduction: DENZO and SCALEPACK (Otwinowski et al., 2003); program(s) used to solve structure: SHELXS97 (Sheldrick, 2008); program(s) used to refine structure: SHELXL2016 (Sheldrick, 2015); molecular graphics: DIAMOND (Brandenburg, 2005). Software used to prepare material for publication: publCIF (Westrip, 2010) for RbGa2AsHAsO46.

Rubidium digallium arsenic(V) hexakis[hydrogen arsenate(V)] (RbGa2AsHAsO46)

Crystal data

$\mathrm{RbGa}_{2} \mathrm{As}\left(\mathrm{HAsO}_{4}\right)_{6}$

$M_{r}=1139.40$

Trigonal, $R \overline{3} c: H$

$a=8.491$ (1) $\AA$

$c=50.697(11) \AA$

$V=3165.4(10) \AA^{3}$

$Z=6$

$F(000)=3168$

Data collection

Nonius KappaCCD single-crystal four-circle diffractometer

Radiation source: fine-focus sealed tube $\varphi$ and $\omega$ scans

Absorption correction: multi-scan (SCALEPACK; Otwinowski et al., 2003)

$T_{\min }=0.232, T_{\max }=0.252$

4684 measured reflections

\section{Refinement}

Refinement on $F^{2}$

Least-squares matrix: full

$R\left[F^{2}>2 \sigma\left(F^{2}\right)\right]=0.014$

$w R\left(F^{2}\right)=0.034$

$S=1.13$

1287 reflections

65 parameters

2 restraints

Primary atom site location: structure-invariant direct methods

Secondary atom site location: difference Fourier map
$D_{\mathrm{x}}=3.586 \mathrm{Mg} \mathrm{m}^{-3}$

Mo $K \alpha$ radiation, $\lambda=0.71073 \AA$

Cell parameters from 2570 reflections

$\theta=2.0-32.6^{\circ}$

$\mu=15.85 \mathrm{~mm}^{-1}$

$T=293 \mathrm{~K}$

Large pseudo-octahedra, colourless

$0.13 \times 0.12 \times 0.12 \mathrm{~mm}$

1287 independent reflections

1196 reflections with $I>2 \sigma(I)$

$R_{\text {int }}=0.016$

$\theta_{\text {max }}=32.5^{\circ}, \theta_{\min }=2.4^{\circ}$

$h=-12 \rightarrow 12$

$k=-10 \rightarrow 10$

$l=-75 \rightarrow 76$

Hydrogen site location: difference Fourier map

All $\mathrm{H}$-atom parameters refined

$w=1 /\left[\sigma^{2}\left(F_{\mathrm{o}}^{2}\right)+(0.0136 P)^{2}+10.4877 P\right]$ where $P=\left(F_{\mathrm{o}}^{2}+2 F_{\mathrm{c}}^{2}\right) / 3$

$(\Delta / \sigma)_{\max }=0.008$

$\Delta \rho_{\max }=0.75$ e $\AA^{-3}$

$\Delta \rho_{\min }=-0.83$ e $\AA^{-3}$

Extinction correction: SHELXL2016

(Sheldrick, 2015),

$\mathrm{Fc}^{*}=\mathrm{kFc}\left[1+0.001 \mathrm{xFc}^{2} \lambda^{3} / \sin (2 \theta)\right]^{-1 / 4}$

Extinction coefficient: 0.000271 (17) 


\section{Special details}

Geometry. All esds (except the esd in the dihedral angle between two 1.s. planes) are estimated using the full covariance matrix. The cell esds are taken into account individually in the estimation of esds in distances, angles and torsion angles; correlations between esds in cell parameters are only used when they are defined by crystal symmetry. An approximate (isotropic) treatment of cell esds is used for estimating esds involving l.s. planes.

Fractional atomic coordinates and isotropic or equivalent isotropic displacement parameters $\left(\hat{A}^{2}\right)$

\begin{tabular}{llllll}
\hline & $x$ & $y$ & $z$ & $U_{\text {iso }} * / U_{\text {eq }}$ & Occ. $(<1)$ \\
\hline Rb1A & 0.000000 & 0.000000 & 0.750000 & $0.055(3)$ & $0.669(3)$ \\
Rb1B & 0.000000 & $-0.048(2)$ & 0.750000 & $0.0359(11)$ & $0.1103(9)$ \\
Ga1 & 0.333333 & 0.666667 & $0.75604(2)$ & $0.00736(6)$ & \\
As1 & 0.333333 & 0.666667 & 0.666667 & $0.00627(7)$ & \\
As2 & $-0.44400(2)$ & $-0.40772(2)$ & $0.71144(2)$ & $0.00735(5)$ & \\
O1 & $0.40249(16)$ & $-0.46597(15)$ & $0.68629(2)$ & $0.01016(19)$ & \\
O2 & $-0.45254(15)$ & $-0.27043(15)$ & $0.73416(2)$ & $0.01025(19)$ & \\
O3 & $-0.22887(17)$ & $-0.28592(18)$ & $0.69874(3)$ & $0.0194(3)$ & \\
O4 & $0.48817(15)$ & $-0.12495(15)$ & $0.77869(2)$ & $0.0108(2)$ & \\
H & $-0.176(4)$ & $-0.337(4)$ & $0.7027(6)$ & $0.046(9)^{*}$ & \\
\hline
\end{tabular}

Atomic displacement parameters $\left(\AA^{2}\right)$

\begin{tabular}{lllllll}
\hline & $U^{11}$ & $U^{22}$ & $U^{33}$ & $U^{12}$ & $U^{13}$ & $U^{23}$ \\
\hline $\mathrm{Rb} 1 \mathrm{~A}$ & $0.050(4)$ & $0.050(4)$ & $0.066(2)$ & $0.0251(18)$ & 0.000 & 0.000 \\
$\mathrm{Rb} 1 \mathrm{~B}$ & $0.024(4)$ & $0.034(4)$ & $0.046(4)$ & $0.012(2)$ & $0.0044(14)$ & $0.0022(7)$ \\
$\mathrm{Ga} 1$ & $0.00807(8)$ & $0.00807(8)$ & $0.00596(12)$ & $0.00403(4)$ & 0.000 & 0.000 \\
$\mathrm{As} 1$ & $0.00747(10)$ & $0.00747(10)$ & $0.00389(14)$ & $0.00373(5)$ & 0.000 & 0.000 \\
$\mathrm{As} 2$ & $0.00844(7)$ & $0.00803(7)$ & $0.00670(7)$ & $0.00495(5)$ & $0.00010(5)$ & $0.00053(5)$ \\
$\mathrm{O} 1$ & $0.0138(5)$ & $0.0110(5)$ & $0.0078(4)$ & $0.0077(4)$ & $-0.0031(4)$ & $0.0000(4)$ \\
O2 & $0.0109(5)$ & $0.0099(5)$ & $0.0098(5)$ & $0.0051(4)$ & $0.0005(4)$ & $-0.0022(4)$ \\
O3 & $0.0121(5)$ & $0.0203(6)$ & $0.0264(7)$ & $0.0084(5)$ & $0.0076(5)$ & $0.0075(5)$ \\
O4 & $0.0124(5)$ & $0.0095(5)$ & $0.0125(5)$ & $0.0070(4)$ & $-0.0030(4)$ & $-0.0042(4)$ \\
\hline
\end{tabular}

Geometric parameters $\left(\AA,{ }^{o}\right)$

\begin{tabular}{|c|c|c|c|}
\hline $\mathrm{Rb} 1 \mathrm{~A}-\mathrm{Rb} 1 \mathrm{~B}^{\mathrm{i}}$ & $0.41(2)$ & $\mathrm{Rb} 1 \mathrm{~B}-\mathrm{O} 2$ & $3.4234(12)$ \\
\hline $\mathrm{Rb} 1 \mathrm{~A}-\mathrm{Rb} 1 \mathrm{~B}^{\mathrm{ii}}$ & $0.41(2)$ & $\mathrm{Rb} 1 \mathrm{~B}-\mathrm{O} 2^{\mathrm{iv}}$ & $3.4234(12)$ \\
\hline $\mathrm{Rb} 1 \mathrm{~A}-\mathrm{O} 3^{\mathrm{iii}}$ & $3.4212(16)$ & $\mathrm{Rb} 1 \mathrm{~B}-\mathrm{O} 3^{\mathrm{iii}}$ & $3.694(14)$ \\
\hline $\mathrm{Rb} 1 \mathrm{~A}-\mathrm{O}^{\mathrm{iv}}$ & $3.4212(16)$ & $\mathrm{Rb} 1 \mathrm{~B}-\mathrm{O} 3^{\mathrm{ii}}$ & $3.694(14)$ \\
\hline $\mathrm{Rb} 1 \mathrm{~A}-\mathrm{O} 3^{\mathrm{ii}}$ & $3.4212(15)$ & $\mathrm{Rb} 1 \mathrm{~B}-\mathrm{O} 2^{\mathrm{ii}}$ & $3.810(18)$ \\
\hline $\mathrm{Rb} 1 \mathrm{~A}-\mathrm{O}^{\mathrm{v}}$ & $3.4212(16)$ & $\mathrm{Rb} 1 \mathrm{~B}-\mathrm{O} 2^{\mathrm{iii}}$ & $3.810(18)$ \\
\hline $\mathrm{Rb} 1 \mathrm{~A}-\mathrm{O} 3^{\mathrm{i}}$ & $3.4212(15)$ & $\mathrm{Rb} 1 \mathrm{~B}-\mathrm{O} 4^{\mathrm{vi}}$ & $3.819(18)$ \\
\hline $\mathrm{Rb} 1 \mathrm{~A}-\mathrm{O} 3$ & $3.4213(16)$ & $\mathrm{Rb} 1 \mathrm{~B}-\mathrm{O} 4^{\mathrm{vii}}$ & $3.819(18)$ \\
\hline $\mathrm{Rb} 1 \mathrm{~A}-\mathrm{O} 2^{\mathrm{iv}}$ & $3.4438(12)$ & $\mathrm{Rb} 1 \mathrm{~B}-\mathrm{As} 2^{\mathrm{v}}$ & $3.934(8)$ \\
\hline $\mathrm{Rb} 1 \mathrm{~A}-\mathrm{O} 2^{\mathrm{ii}}$ & $3.4438(12)$ & $\mathrm{Rb} 1 \mathrm{~B}-\mathrm{As} 2^{\mathrm{i}}$ & $3.934(8)$ \\
\hline $\mathrm{Rb} 1 \mathrm{~A}-\mathrm{O} 2$ & $3.4438(12)$ & $\mathrm{Ga} 1-\mathrm{O} 4^{\mathrm{viii}}$ & $1.9623(12)$ \\
\hline $\mathrm{Rb} 1 \mathrm{~A}-\mathrm{O} 2^{\mathrm{v}}$ & $3.4438(12)$ & $\mathrm{Ga} 1-\mathrm{O} 4^{\mathrm{i}}$ & $1.9623(11)$ \\
\hline $\mathrm{Rb} 1 \mathrm{~A}-\mathrm{O} 2^{\mathrm{iii}}$ & $3.4438(12)$ & $\mathrm{Ga} 1-\mathrm{O} 4^{\mathrm{ix}}$ & $1.9623(11)$ \\
\hline
\end{tabular}


$\mathrm{Rb} 1 \mathrm{~A}-\mathrm{O} 2^{\mathrm{i}}$

$\mathrm{Rb} 1 \mathrm{~A}-\mathrm{As} 2^{\mathrm{iv}}$

$\mathrm{Rb} 1 \mathrm{~A}-\mathrm{As} 2^{\mathrm{iii}}$

$\mathrm{Rb} 1 \mathrm{~A}-\mathrm{As} 2^{\mathrm{v}}$

$\mathrm{Rb} 1 \mathrm{~A}-\mathrm{As} 2^{\mathrm{ii}}$

Rb1B-Rb1B ${ }^{i}$

$\mathrm{Rb} 1 \mathrm{~B}-\mathrm{Rb}^{\mathrm{ii}}$

$\mathrm{Rb} 1 \mathrm{~B}-\mathrm{O} 2^{\mathrm{v}}$

$\mathrm{Rb} 1 \mathrm{~B}-\mathrm{O} 2^{\mathrm{i}}$

$\mathrm{Rb} 1 \mathrm{~B}-\mathrm{O} 3^{\text {iv }}$

$\mathrm{Rb} 1 \mathrm{~B}-\mathrm{O} 3$

$\mathrm{Rb} 1 \mathrm{~B}-\mathrm{O} 3^{\mathrm{v}}$

$\mathrm{Rb} 1 \mathrm{~B}-\mathrm{O} 3^{\mathrm{i}}$

$R b 1 B^{i}-R b 1 A-R b 1 B^{i i}$

$\mathrm{Rb} 1 \mathrm{~B}^{\mathrm{i}}-\mathrm{Rb} 1 \mathrm{~A}-\mathrm{O} 3^{\mathrm{iii}}$

$\mathrm{Rb} 1 \mathrm{~B}^{\mathrm{ii}}-\mathrm{Rb} 1 \mathrm{~A}-\mathrm{O} 3^{\mathrm{iii}}$

$\mathrm{Rb} 1 \mathrm{~B}^{\mathrm{i}}-\mathrm{Rb} 1 \mathrm{~A}-\mathrm{O}^{\mathrm{iv}}$

$\mathrm{Rb} 1 \mathrm{~B}^{\mathrm{ii}}-\mathrm{Rb} 1 \mathrm{~A}-\mathrm{O}^{3 \mathrm{iv}}$

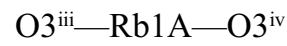

$\mathrm{Rb} 1 \mathrm{~B}-\mathrm{Rb} 1 \mathrm{~A}-\mathrm{O} 3^{\mathrm{ii}}$

$\mathrm{Rb} 1 \mathrm{~B}^{\mathrm{ii}}-\mathrm{Rb} 1 \mathrm{~A}-\mathrm{O} 3^{\mathrm{ii}}$

$\mathrm{O}^{\mathrm{iii}}-\mathrm{Rb} 1 \mathrm{~A}-\mathrm{O} 3^{\mathrm{ii}}$

$\mathrm{O}^{\mathrm{iv}}-\mathrm{Rb} 1 \mathrm{~A}-\mathrm{O} 3^{\mathrm{ii}}$

$\mathrm{Rb} 1 \mathrm{~B}^{\mathrm{i}}-\mathrm{Rb} 1 \mathrm{~A}-\mathrm{O}^{\mathrm{v}}$

$\mathrm{Rb} 1 \mathrm{~B}^{\mathrm{ii}}-\mathrm{Rb} 1 \mathrm{~A}-\mathrm{O}^{\mathrm{v}}$

$\mathrm{O}^{\mathrm{iii}}-\mathrm{Rb} 1 \mathrm{~A}-\mathrm{O}^{\mathrm{v}}$

$\mathrm{O} 3^{\mathrm{iv}}-\mathrm{Rb} 1 \mathrm{~A}-\mathrm{O} 3^{\mathrm{v}}$

$\mathrm{O} 3^{\mathrm{ii}}-\mathrm{Rb} 1 \mathrm{~A}-\mathrm{O}^{\mathrm{v}}$

$\mathrm{Rb} 1 \mathrm{~B}^{\mathrm{i}}-\mathrm{Rb} 1 \mathrm{~A}-\mathrm{O} 3^{\mathrm{i}}$

$\mathrm{Rb} 1 \mathrm{~B}^{\mathrm{ii}}-\mathrm{Rb} 1 \mathrm{~A}-\mathrm{O} 3^{\mathrm{i}}$

$\mathrm{O}^{\mathrm{iii}}-\mathrm{Rb} 1 \mathrm{~A}-\mathrm{O}^{\mathrm{i}}$

$\mathrm{O} 3^{\mathrm{iv}}-\mathrm{Rb} 1 \mathrm{~A}-\mathrm{O} 3^{\mathrm{i}}$

$\mathrm{O} 3^{\mathrm{ii}}-\mathrm{Rb} 1 \mathrm{~A}-\mathrm{O} 3^{\mathrm{i}}$

$\mathrm{O}^{\mathrm{v}}-\mathrm{Rb} 1 \mathrm{~A}-\mathrm{O} 3^{\mathrm{i}}$

$\mathrm{Rb} 1 \mathrm{~B}-\mathrm{Rb} 1 \mathrm{~A}-\mathrm{O} 3$

$\mathrm{Rb} 1 \mathrm{~B}^{\mathrm{ii}}-\mathrm{Rb} 1 \mathrm{~A}-\mathrm{O} 3$

$\mathrm{O} 3{ }^{\mathrm{iii}}-\mathrm{Rb} 1 \mathrm{~A}-\mathrm{O} 3$

$\mathrm{O}^{\mathrm{iv}}-\mathrm{Rb} 1 \mathrm{~A}-\mathrm{O} 3$

$\mathrm{O} 3{ }^{\mathrm{ii}}-\mathrm{Rb} 1 \mathrm{~A}-\mathrm{O} 3$

$\mathrm{O} 3{ }^{\mathrm{v}}-\mathrm{Rb} 1 \mathrm{~A}-\mathrm{O} 3$

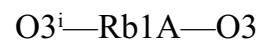

$\mathrm{Rb} 1 \mathrm{~B}-\mathrm{Rb} 1 \mathrm{~A}-\mathrm{O} 2^{\mathrm{iv}}$

$\mathrm{Rb} 1 \mathrm{~B}^{\mathrm{ii}}-\mathrm{Rb} 1 \mathrm{~A}-\mathrm{O} 2^{\mathrm{iv}}$

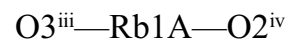

$\mathrm{O} 3^{\text {iv }}-\mathrm{Rb} 1 \mathrm{~A}-\mathrm{O} 2^{\text {iv }}$

$\mathrm{O} 3^{\mathrm{ii}}-\mathrm{Rb} 1 \mathrm{~A}-\mathrm{O} 2^{\mathrm{iv}}$

$\mathrm{O} 3^{\mathrm{v}}-\mathrm{Rb} 1 \mathrm{~A}-\mathrm{O} 2^{\text {iv }}$
3.4438 (12)

4.1192 (5)

4.1192 (5)

$4.1192(5)$

4.1192 (4)

$0.70(3)$

0.70 (3)

3.136 (14)

3.136 (14)

3.269 (6)

3.269 (6)

$3.358(2)$

3.358 (2)

$120.00(7)$

64.81 (2)

77.69 (5)

77.69 (2)

$129.70(3)$

68.57 (4)

77.69 (2)

$64.81(5)$

$100.59(5)$

155.38 (5)

$129.70(2)$

64.81 (5)

68.57 (4)

68.57 (4)

$129.62(5)$

64.81 (2)

129.70 (3)

$129.62(5)$

$100.59(5)$

68.57 (4)

155.38 (4)

$129.70(2)$

77.69 (5)

155.38 (4)

$129.62(5)$

68.57 (4)

$100.59(5)$

68.57 (4)

38.519 (18)

$153.03(7)$

76.93 (3)

45.66 (3)

111.42 (3)

113.17 (3)
$\mathrm{Ga} 1-\mathrm{O} 2^{\mathrm{x}}$

$\mathrm{Ga} 1-\mathrm{O} 2^{\mathrm{ii}}$

$\mathrm{Ga} 1-\mathrm{O} 2^{\mathrm{xi}}$

As $1-O 1^{\text {xii }}$

As $1-O 1^{\text {xiii }}$

As $1-\mathrm{O}^{\mathrm{xiv}}$

As $1-O 1^{i}$

As $1-O 1^{\text {ix }}$

As $1-O 1^{\text {viii }}$

As2- 02

As $2-O 4^{\text {iv }}$

As $2-\mathrm{O}^{\mathrm{xv}}$

As2-O3

1.9625 (11)

1.9625 (11)

1.9625 (11)

1.8067 (11)

1.8068 (11)

1.8068 (11)

1.8068 (11)

1.8068 (11)

1.8068 (11)

1.6658 (11)

$1.6670(11)$

1.7100 (11)

1.7122 (13)

$\mathrm{Rb} 1 \mathrm{~B}^{\mathrm{ii}}-\mathrm{Rb} 1 \mathrm{~B}-\mathrm{O} 4^{\text {vii }}$

$153.31(7)$

53.4 (3)

45.1 (2)

44.49 (19)

$98.0(5)$

76.7 (3)

93.3 (3)

109.4 (4)

$71.6(2)$

$109.34(9)$

$157.2(3)$

$144.11(3)$

144.95 (3)

53.5 (3)

137.8 (2)

88.8 (3)

24.03 (3)

107.9 (5)

82.2 (2)

82.5 (2)

$25.63(7)$

$145.3(5)$

$51.65(8)$

$128.9(3)$

$91.62(8)$

$123.66(15)$

138.7 (4)

90.34 (14)

$68.6(3)$

67.9 (3)

$88.8(2)$

$137.8(2)$

$107.9(5)$

24.03 (3) 


\begin{tabular}{|c|c|c|c|}
\hline $\mathrm{O} 3^{\mathrm{i}}-\mathrm{Rb} 1 \mathrm{~A}-\mathrm{O} 2^{\mathrm{iv}}$ & $63.90(3)$ & $\mathrm{O} 3^{\mathrm{iv}}-\mathrm{Rb} 1 \mathrm{~B}-\mathrm{As} 2^{\mathrm{i}}$ & $82.5(2)$ \\
\hline $\mathrm{O} 3-\mathrm{Rb} 1 \mathrm{~A}-\mathrm{O} 2^{\mathrm{iv}}$ & $127.31(3)$ & $\mathrm{O} 3-\mathrm{Rb} 1 \mathrm{~B}-\mathrm{As} 2^{\mathrm{i}}$ & $82.2(2)$ \\
\hline $\mathrm{Rb} 1 \mathrm{~B}-\mathrm{Rb} 1 \mathrm{~A}-\mathrm{O} 2^{\mathrm{ii}}$ & $38.519(18)$ & $\mathrm{O} 3^{\mathrm{v}}-\mathrm{Rb} 1 \mathrm{~B}-\mathrm{As} 2^{\mathrm{i}}$ & $145.3(5)$ \\
\hline $\mathrm{Rb} 1 \mathrm{~B}{ }^{\mathrm{ii}}-\mathrm{Rb} 1 \mathrm{~A}-\mathrm{O} 2^{\mathrm{ii}}$ & $83.75(7)$ & $\mathrm{O} 3^{\mathrm{i}}-\mathrm{Rb} 1 \mathrm{~B}-\mathrm{As} 2^{\mathrm{i}}$ & $25.63(7)$ \\
\hline 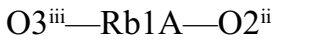 & $63.90(3)$ & $\mathrm{O} 2-\mathrm{Rb} 1 \mathrm{~B}-\mathrm{As} 2^{\mathrm{i}}$ & $128.9(3)$ \\
\hline $\mathrm{O} 3^{\mathrm{iv}}-\mathrm{Rb} 1 \mathrm{~A}-\mathrm{O} 2^{\mathrm{ii}}$ & $111.42(3)$ & $\mathrm{O} 2^{\mathrm{iv}}-\mathrm{Rb} 1 \mathrm{~B}-\mathrm{As} 2^{\mathrm{i}}$ & $51.65(8)$ \\
\hline 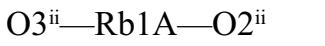 & $45.66(3)$ & $\mathrm{O} 3^{\mathrm{iii}}-\mathrm{Rb} 1 \mathrm{~B}-\mathrm{As} 2^{\mathrm{i}}$ & $123.66(15)$ \\
\hline $\mathrm{O} 3^{\mathrm{v}}-\mathrm{Rb} 1 \mathrm{~A}-\mathrm{O} 2^{\mathrm{ii}}$ & $127.31(3)$ & $\mathrm{O} 3^{\mathrm{ii}}-\mathrm{Rb} 1 \mathrm{~B}-\mathrm{As} 2^{\mathrm{i}}$ & $91.62(8)$ \\
\hline $\mathrm{O} 3^{\mathrm{i}}-\mathrm{Rb} 1 \mathrm{~A}-\mathrm{O} 2^{\mathrm{ii}}$ & $76.93(3)$ & 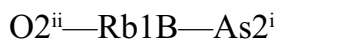 & $90.34(14)$ \\
\hline $\mathrm{O} 3-\mathrm{Rb} 1 \mathrm{~A}-\mathrm{O} 2^{\mathrm{ii}}$ & $113.17(3)$ & $\mathrm{O} 2^{\mathrm{iii}}-\mathrm{Rb} 1 \mathrm{~B}-\mathrm{As} 2^{\mathrm{i}}$ & $138.7(4)$ \\
\hline $\mathrm{O} 2^{\mathrm{iv}}-\mathrm{Rb} 1 \mathrm{~A}-\mathrm{O} 2^{\mathrm{ii}}$ & $77.04(4)$ & $\mathrm{O} 4^{\mathrm{vi}}-\mathrm{Rb} 1 \mathrm{~B}-\mathrm{As} 2^{\mathrm{i}}$ & $67.9(3)$ \\
\hline $\mathrm{Rb} 1 \mathrm{~B}^{\mathrm{i}}-\mathrm{Rb} 1 \mathrm{~A}-\mathrm{O} 2$ & $153.035(19)$ & $\mathrm{O} 4^{\mathrm{vii}}-\mathrm{Rb} 1 \mathrm{~B}-\mathrm{As} 2^{\mathrm{i}}$ & $68.6(3)$ \\
\hline $\mathrm{Rb} 1 \mathrm{~B}^{\mathrm{ii}}-\mathrm{Rb} 1 \mathrm{~A}-\mathrm{O} 2$ & $38.52(7)$ & $A s 2^{v}-R b 1 B-A s 2^{i}$ & $131.0(5)$ \\
\hline 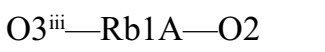 & $111.42(3)$ & 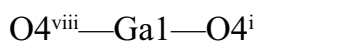 & $89.24(5)$ \\
\hline $\mathrm{O}^{\mathrm{iv}}-\mathrm{Rb} 1 \mathrm{~A}-\mathrm{O} 2$ & $127.31(3)$ & $\mathrm{O} 4^{\mathrm{viii}}-\mathrm{Ga} 1-\mathrm{O} 4^{\mathrm{ix}}$ & $89.24(5)$ \\
\hline $\mathrm{O} 3{ }^{\mathrm{ii}}-\mathrm{Rb} 1 \mathrm{~A}-\mathrm{O} 2$ & $76.93(3)$ & $\mathrm{O} 4^{\mathrm{i}}-\mathrm{Ga} 1-\mathrm{O} 4^{\mathrm{ix}}$ & $89.24(5)$ \\
\hline $\mathrm{O} 3^{\mathrm{v}}-\mathrm{Rb} 1 \mathrm{~A}-\mathrm{O} 2$ & $63.90(3)$ & 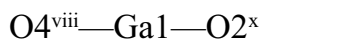 & $91.13(5)$ \\
\hline $\mathrm{O} 3^{\mathrm{i}}-\mathrm{Rb} 1 \mathrm{~A}-\mathrm{O} 2$ & $113.17(3)$ & 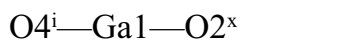 & $88.49(5)$ \\
\hline $\mathrm{O} 3-\mathrm{Rb} 1 \mathrm{~A}-\mathrm{O} 2$ & $45.67(3)$ & $\mathrm{O} 4^{\mathrm{ix}}-\mathrm{Ga} 1-\mathrm{O} 2^{\mathrm{x}}$ & $177.69(5)$ \\
\hline $\mathrm{O} 2^{\mathrm{iv}}-\mathrm{Rb} 1 \mathrm{~A}-\mathrm{O} 2$ & $167.50(4)$ & $\mathrm{O} 4^{\mathrm{viii}}-\mathrm{Ga} 1-\mathrm{O} 2^{\mathrm{ii}}$ & $177.69(5)$ \\
\hline $\mathrm{O} 22^{\mathrm{ii}}-\mathrm{Rb} 1 \mathrm{~A}-\mathrm{O} 2$ & $114.733(13)$ & $\mathrm{O} 4^{\mathrm{i}}-\mathrm{Ga} 1-\mathrm{O} 2^{\mathrm{ii}}$ & $91.13(5)$ \\
\hline $\mathrm{Rb} 1 \mathrm{~B}-\mathrm{Rb} 1 \mathrm{~A}-\mathrm{O} 2^{\mathrm{v}}$ & $153.03(2)$ & $\mathrm{O} 4^{\mathrm{ix}}-\mathrm{Ga} 1-\mathrm{O} 2^{\mathrm{ii}}$ & $88.48(5)$ \\
\hline $\mathrm{Rb} 1 \mathrm{~B}-\mathrm{ii}-\mathrm{Rb} 1 \mathrm{~A}-\mathrm{O} 2^{\mathrm{v}}$ & $83.75(8)$ & $\mathrm{O} 2^{\mathrm{x}}-\mathrm{Ga} 1-\mathrm{O} 2^{\mathrm{ii}}$ & $91.17(5)$ \\
\hline 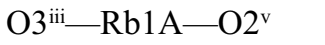 & $113.17(3)$ & $\mathrm{O} 4^{\mathrm{viii}}-\mathrm{Ga} 1-\mathrm{O} 2^{\mathrm{xi}}$ & $88.48(5)$ \\
\hline $\mathrm{O} 3^{\mathrm{iv}}-\mathrm{Rb} 1 \mathrm{~A}-\mathrm{O} 2^{\mathrm{v}}$ & $76.93(3)$ & $\mathrm{O} 4^{\mathrm{i}}-\mathrm{Ga} 1-\mathrm{O} 2^{\mathrm{xi}}$ & $177.69(5)$ \\
\hline 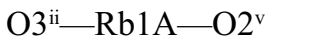 & $127.31(3)$ & $\mathrm{O} 4^{\mathrm{ix}}-\mathrm{Ga} 1-\mathrm{O} 2^{\mathrm{xi}}$ & $91.12(5)$ \\
\hline $\mathrm{O} 3^{\mathrm{v}}-\mathrm{Rb} 1 \mathrm{~A}-\mathrm{O} 2^{\mathrm{v}}$ & $45.66(3)$ & $\mathrm{O} 2^{\mathrm{x}}-\mathrm{Ga} 1-\mathrm{O} 2^{\mathrm{xi}}$ & $91.16(5)$ \\
\hline $\mathrm{O} 3^{\mathrm{i}}-\mathrm{Rb} 1 \mathrm{~A}-\mathrm{O} 2^{\mathrm{v}}$ & $111.42(3)$ & $\mathrm{O} 2^{\mathrm{ii}}-\mathrm{Ga} 1-\mathrm{O} 2^{\mathrm{xi}}$ & $91.16(5)$ \\
\hline $\mathrm{O} 3-\mathrm{Rb} 1 \mathrm{~A}-\mathrm{O} 2^{\mathrm{v}}$ & $63.91(3)$ & $\mathrm{O} 4^{\text {viii }}-\mathrm{Ga} 1-\mathrm{Rb} 1 \mathrm{~B}^{\mathrm{viii}}$ & $82.77(12)$ \\
\hline $\mathrm{O} 2^{\mathrm{iv}}-\mathrm{Rb} 1 \mathrm{~A}-\mathrm{O} 2^{\mathrm{v}}$ & $114.733(13)$ & $\mathrm{O} 4^{\mathrm{i}}-\mathrm{Ga} 1-\mathrm{Rb} \mathrm{B}^{\mathrm{viii}}$ & $55.65(8)$ \\
\hline 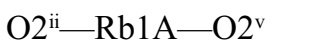 & $167.50(4)$ & $\mathrm{O} 4^{\mathrm{ix}}-\mathrm{Ga} 1-\mathrm{Rb} 1 \mathrm{~B}^{\mathrm{viii}}$ & $143.89(7)$ \\
\hline $\mathrm{O} 2-\mathrm{Rb} 1 \mathrm{~A}-\mathrm{O} 2^{\mathrm{v}}$ & $53.93(4)$ & $\mathrm{O} 2^{\mathrm{x}}-\mathrm{Ga} 1-\mathrm{Rb} 1 \mathrm{~B}^{\mathrm{viii}}$ & $33.99(5)$ \\
\hline $\mathrm{Rb} 1 \mathrm{~B}-\mathrm{Rb} 1 \mathrm{~A}-\mathrm{O} 2^{\mathrm{iii}}$ & 83.749 (19) & $\mathrm{O} 2^{\mathrm{ii}}-\mathrm{Ga} 1-\mathrm{Rb} 1 \mathrm{~B}^{\mathrm{viii}}$ & $99.31(13)$ \\
\hline $\mathrm{Rb} 1 \mathrm{~B}^{\mathrm{ii}}-\mathrm{Rb} 1 \mathrm{~A}-\mathrm{O} 2^{\mathrm{iii}}$ & $38.52(7)$ & $\mathrm{O} 2^{\mathrm{xi}}-\mathrm{Ga} 1-\mathrm{Rb} 1 \mathrm{~B}^{\mathrm{viii}}$ & $123.61(10)$ \\
\hline $\mathrm{O} 3^{\mathrm{iii}}-\mathrm{Rb} 1 \mathrm{~A}-\mathrm{O} 2^{\mathrm{iii}}$ & $45.66(3)$ & $\mathrm{O} 4^{\mathrm{viii}}-\mathrm{Ga} 1-\mathrm{Rb}_{1} \mathrm{~B}^{\mathrm{i}}$ & $143.89(6)$ \\
\hline $\mathrm{O} 3^{\mathrm{iv}}-\mathrm{Rb} 1 \mathrm{~A}-\mathrm{O} 2^{\mathrm{iii}}$ & $113.17(3)$ & $\mathrm{O} 4^{\mathrm{i}}-\mathrm{Ga} 1-\mathrm{Rb} 1 \mathrm{~B}^{\mathrm{i}}$ & $82.77(12)$ \\
\hline 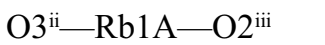 & $63.90(3)$ & $\mathrm{O} 4^{\mathrm{ix}}-\mathrm{Ga} 1-\mathrm{Rb} 1 \mathrm{~B}^{\mathrm{i}}$ & $55.65(8)$ \\
\hline $\mathrm{O} 3^{\mathrm{v}}-\mathrm{Rb} 1 \mathrm{~A}-\mathrm{O} 2^{\mathrm{iii}}$ & $76.93(3)$ & $\mathrm{O} 2^{\mathrm{x}}-\mathrm{Ga} 1-\mathrm{Rb} 1 \mathrm{~B}^{\mathrm{i}}$ & $123.61(9)$ \\
\hline $\mathrm{O} 3^{\mathrm{i}}-\mathrm{Rb} 1 \mathrm{~A}-\mathrm{O} 2^{\mathrm{iii}}$ & $127.31(3)$ & $\mathrm{O} 2^{\mathrm{ii}}-\mathrm{Ga} 1-\mathrm{Rb} 1 \mathrm{~B}^{\mathrm{i}}$ & $33.99(5)$ \\
\hline $\mathrm{O} 3-\mathrm{Rb} 1 \mathrm{~A}-\mathrm{O} 2^{\mathrm{iii}}$ & $111.42(3)$ & $\mathrm{O} 2^{\mathrm{xi}}-\mathrm{Ga} 1-\mathrm{Rb} 1 \mathrm{~B}^{\mathrm{i}}$ & $99.31(12)$ \\
\hline $\mathrm{O} 2^{\mathrm{iv}}-\mathrm{Rb} 1 \mathrm{~A}-\mathrm{O} 2^{\mathrm{iii}}$ & $114.732(14)$ & $\mathrm{Rb} 1 \mathrm{~B}^{\mathrm{viii}}-\mathrm{Ga} 1-\mathrm{Rb} 1 \mathrm{~B}^{\mathrm{i}}$ & $119.554(12)$ \\
\hline $\mathrm{O} 2^{\mathrm{ii}}-\mathrm{Rb} 1 \mathrm{~A}-\mathrm{O} 2^{\mathrm{iii}}$ & $53.93(4)$ & $\mathrm{O} 4^{\mathrm{viii}}-\mathrm{Ga} 1-\mathrm{Rb} 1 \mathrm{~B}^{\mathrm{ix}}$ & $55.65(10)$ \\
\hline $\mathrm{O} 2-\mathrm{Rb} 1 \mathrm{~A}-\mathrm{O} 2^{\mathrm{iii}}$ & $77.04(4)$ & $\mathrm{O} 4-\mathrm{Ga} 1-\mathrm{Rb} 1 \mathrm{~B}^{\mathrm{i} x}$ & $143.89(7)$ \\
\hline $\mathrm{O} 2^{\mathrm{v}}-\mathrm{Rb} 1 \mathrm{~A}-\mathrm{O} 2^{\mathrm{iii}}$ & $114.731(14)$ & $\mathrm{O} 4^{\mathrm{ix}}-\mathrm{Ga} 1-\mathrm{Rb} 1 \mathrm{~B}^{\mathrm{ix}}$ & $82.77(13)$ \\
\hline $\mathrm{Rb} 1 \mathrm{~B}^{\mathrm{i}}-\mathrm{Rb} 1 \mathrm{~A}-\mathrm{O} 2^{\mathrm{i}}$ & $83.749(19)$ & $\mathrm{O} 2^{\mathrm{x}}-\mathrm{Ga} 1-\mathrm{Rb} 1 \mathrm{~B}^{\mathrm{ix}}$ & $99.31(14)$ \\
\hline $\mathrm{Rb} 1 \mathrm{~B}^{\mathrm{ii}}-\mathrm{Rb} 1 \mathrm{~A}-\mathrm{O} 2^{\mathrm{i}}$ & $153.03(7)$ & $\mathrm{O} 2^{\mathrm{ii}}-\mathrm{Ga} 1-\mathrm{Rb} 1 \mathrm{~B}^{\mathrm{ix}}$ & $123.61(9)$ \\
\hline 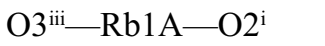 & $127.31(3)$ & $\mathrm{O} 2^{\mathrm{xi}}-\mathrm{Ga} 1-\mathrm{Rb} 1 \mathrm{~B}^{\mathrm{ix}}$ & $33.99(6)$ \\
\hline $\mathrm{O} 3^{\mathrm{iv}}-\mathrm{Rb} 1 \mathrm{~A}-\mathrm{O} 2^{\mathrm{i}}$ & $63.90(3)$ & $\mathrm{Rb}_{1 \mathrm{~B}} \mathrm{viii}^{\mathrm{vii}} \mathrm{Ga} 1-\mathrm{Rb} 1 \mathrm{~B}^{\mathrm{ix}}$ & $119.554(7)$ \\
\hline
\end{tabular}




\begin{tabular}{|c|c|c|c|}
\hline $\mathrm{O} 3^{\mathrm{ii}}-\mathrm{Rb} 1 \mathrm{~A}-\mathrm{O} 2^{\mathrm{i}}$ & $113.17(3)$ & $\mathrm{Rb} 1 \mathrm{~B}^{\mathrm{i}}-\mathrm{Ga} 1-\mathrm{Rb} \mathrm{B}^{\mathrm{ix}}$ & $119.554(2)$ \\
\hline $\mathrm{O}^{\mathrm{v}}-\mathrm{Rb} 1 \mathrm{~A}-\mathrm{O}^{2}{ }^{\mathrm{i}}$ & $111.42(3)$ & $\mathrm{O} 4^{\mathrm{viii}}-\mathrm{Ga} 1-\mathrm{Rb}_{1} \mathrm{~A}^{\mathrm{viii}}$ & $80.57(3)$ \\
\hline $\mathrm{O} 3^{\mathrm{i}}-\mathrm{Rb} 1 \mathrm{~A}-\mathrm{O} 2^{\mathrm{i}}$ & $45.66(3)$ & $\mathrm{O} 4$ - $\mathrm{Ga} 1-\mathrm{Rb}_{1} \mathrm{~A}^{\mathrm{viii}}$ & $57.14(3)$ \\
\hline $\mathrm{O} 3-\mathrm{Rb} 1 \mathrm{~A}-\mathrm{O} 2^{\mathrm{i}}$ & $76.93(3)$ & $\mathrm{O} 4^{\mathrm{ix}}-\mathrm{Ga} 1-\mathrm{Rb}_{1} \mathrm{~A}^{\mathrm{viii}}$ & $144.67(3)$ \\
\hline $\mathrm{O} 2^{\mathrm{iv}}-\mathrm{Rb} 1 \mathrm{~A}-\mathrm{O} 2^{\mathrm{i}}$ & $53.93(4)$ & $\mathrm{O} 2^{\mathrm{x}}-\mathrm{Ga} 1-\mathrm{Rb} 1 \mathrm{~A}^{\mathrm{viii}}$ & $33.28(3)$ \\
\hline 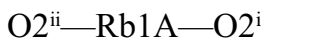 & $114.732(13)$ & $\mathrm{O} 2^{\mathrm{ii}}-\mathrm{Ga} 1-\mathrm{Rb}_{1} \mathrm{~A}^{\mathrm{viii}}$ & $101.54(3)$ \\
\hline $\mathrm{O} 2-\mathrm{Rb} 1 \mathrm{~A}-\mathrm{O} 2^{\mathrm{i}}$ & $114.732(13)$ & $\mathrm{O} 2^{\mathrm{xi}}-\mathrm{Ga} 1-\mathrm{Rb} 1 \mathrm{~A}^{\mathrm{viii}}$ & $122.02(3)$ \\
\hline $\mathrm{O} 2^{\mathrm{v}}-\mathrm{Rb} 1 \mathrm{~A}-\mathrm{O} 2^{\mathrm{i}}$ & $77.04(4)$ & 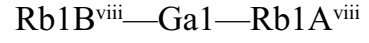 & $2.56(14)$ \\
\hline $\mathrm{O} 2^{\mathrm{iii}}-\mathrm{Rb} 1 \mathrm{~A}-\mathrm{O} 2^{\mathrm{i}}$ & $167.50(4)$ & $\mathrm{Rb} 1 \mathrm{~B}-\mathrm{Ga} 1-\mathrm{Rb} 1 \mathrm{~A}^{\mathrm{v} i i i}$ & $122.12(13)$ \\
\hline $\mathrm{Rb} 1 \mathrm{~B}^{\mathrm{i}}-\mathrm{Rb} 1 \mathrm{~A}-\mathrm{As} 2^{\mathrm{iv}}$ & $60.329(4)$ & $\mathrm{Rb} 1 \mathrm{~B}^{\mathrm{ix}}-\mathrm{Ga} 1-\mathrm{Rb} 1 \mathrm{~A}^{\mathrm{viii}}$ & $117.05(14)$ \\
\hline $\mathrm{Rb} 1 \mathrm{~B}^{\mathrm{ii}}-\mathrm{Rb} 1 \mathrm{~A}-\mathrm{As} 2^{\mathrm{iv}}$ & $151.382(12)$ & $\mathrm{O} 4^{\mathrm{vii}}-\mathrm{Ga} 1-\mathrm{Rb} 1 \mathrm{~A}$ & $144.67(3)$ \\
\hline 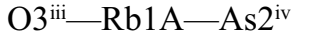 & $77.70(2)$ & $\mathrm{O} 4 \mathrm{i}-\mathrm{Ga} 1-\mathrm{Rb} 1 \mathrm{~A}$ & $80.57(3)$ \\
\hline $\mathrm{O}^{\mathrm{iv}}-\mathrm{Rb} 1 \mathrm{~A}-\mathrm{As} 2^{\mathrm{iv}}$ & $24.04(2)$ & $\mathrm{O} 4^{\mathrm{ix}}-\mathrm{Ga} 1-\mathrm{Rb} 1 \mathrm{~A}$ & $57.14(3)$ \\
\hline $\mathrm{O} 3^{\mathrm{ii}}-\mathrm{Rb} 1 \mathrm{~A}-\mathrm{As} 2^{\mathrm{iv}}$ & $134.60(2)$ & $\mathrm{O} 2{ }^{\mathrm{x}}-\mathrm{Ga} 1-\mathrm{Rb} 1 \mathrm{~A}$ & $122.02(3)$ \\
\hline $\mathrm{O}^{\mathrm{v}}-\mathrm{Rb} 1 \mathrm{~A}-\mathrm{As} 2^{\mathrm{iv}}$ & $92.58(3)$ & $\mathrm{O} 2^{\mathrm{ii}}-\mathrm{Ga} 1-\mathrm{Rb} 1 \mathrm{~A}$ & $33.28(3)$ \\
\hline $\mathrm{O} 33^{\mathrm{i}}-\mathrm{Rb} 1 \mathrm{~A}-\mathrm{As} 2^{\mathrm{iv}}$ & $77.98(3)$ & $\mathrm{O} 2^{\mathrm{xi}}-\mathrm{Ga} 1-\mathrm{Rb} 1 \mathrm{~A}$ & $101.53(3)$ \\
\hline $\mathrm{O} 3-\mathrm{Rb} 1 \mathrm{~A}-\mathrm{As} 2^{\mathrm{iv}}$ & $125.97(2)$ & $\mathrm{Rb} 1 \mathrm{~B}^{\mathrm{viii}}-\mathrm{Ga} 1-\mathrm{Rb} 1 \mathrm{~A}$ & $117.05(14)$ \\
\hline $\mathrm{O} 2^{\mathrm{iv}}-\mathrm{Rb} 1 \mathrm{~A}-\mathrm{As} 2^{\mathrm{iv}}$ & $23.324(18)$ & $\mathrm{Rb} 1 \mathrm{~B}^{\mathrm{i}}-\mathrm{Ga} 1-\mathrm{Rb} 1 \mathrm{~A}$ & $2.56(13)$ \\
\hline $\mathrm{O} 2^{\mathrm{ii}}-\mathrm{Rb} 1 \mathrm{~A}-\mathrm{As} 2^{\mathrm{iv}}$ & $98.261(18)$ & $\mathrm{Rb} 1 \mathrm{~B}^{\mathrm{ix}}-\mathrm{Ga} 1-\mathrm{Rb} 1 \mathrm{~A}$ & $122.12(13)$ \\
\hline $\mathrm{O} 2-\mathrm{Rb} 1 \mathrm{~A}-\mathrm{As} 2^{\mathrm{iv}}$ & $146.607(19)$ & $\mathrm{Rb} 1 \mathrm{~A}^{\mathrm{viii}}-\mathrm{Ga} 1-\mathrm{Rb} 1 \mathrm{~A}$ & $119.614(1)$ \\
\hline $\mathrm{O} 2^{\mathrm{v}}-\mathrm{Rb} 1 \mathrm{~A}-\mathrm{As} 2^{\mathrm{iv}}$ & $92.721(19)$ & 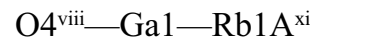 & $57.14(3)$ \\
\hline 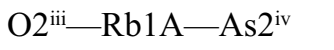 & $122.596(19)$ & $\mathrm{O} 4 \mathrm{i}-\mathrm{Ga} 1-\mathrm{Rb} 1 \mathrm{~A}^{\mathrm{xi}}$ & $144.67(4)$ \\
\hline $\mathrm{O} 2^{\mathrm{i}}-\mathrm{Rb} 1 \mathrm{~A}-\mathrm{As} 2^{\mathrm{iv}}$ & $49.72(2)$ & $\mathrm{O} 4^{\mathrm{ix}}-\mathrm{Ga} 1-\mathrm{Rb} 1 \mathrm{~A}^{\mathrm{xi}}$ & $80.57(3)$ \\
\hline $\mathrm{Rb} 1 \mathrm{~B}^{\mathrm{i}}-\mathrm{Rb} 1 \mathrm{~A}-\mathrm{As} 2^{\mathrm{iii}}$ & $67.492(4)$ & $\mathrm{O} 2^{\mathrm{x}}-\mathrm{Ga} 1-\mathrm{Rb} 1 \mathrm{~A}^{\mathrm{xi}}$ & $101.53(3)$ \\
\hline $\mathrm{Rb} 1 \mathrm{~B}^{\mathrm{ii}}-\mathrm{Rb} 1 \mathrm{~A}-\mathrm{As} 2^{\mathrm{iii}}$ & $60.33(6)$ & $\mathrm{O} 2^{\mathrm{ii}}-\mathrm{Ga} 1-\mathrm{Rb} 1 \mathrm{~A}^{\mathrm{xi}}$ & $122.02(3)$ \\
\hline $\mathrm{O} 3^{\mathrm{iii}}-\mathrm{Rb} 1 \mathrm{~A}-\mathrm{As} 2^{\mathrm{iii}}$ & $24.04(2)$ & $\mathrm{O} 2^{\mathrm{xi}}-\mathrm{Ga} 1-\mathrm{Rb} 1 \mathrm{~A}^{\mathrm{xi}}$ & $33.28(3)$ \\
\hline $\mathrm{O} 3^{\mathrm{iv}}-\mathrm{Rb} 1 \mathrm{~A}-\mathrm{As} 2^{\mathrm{iii}}$ & $92.58(3)$ & $\mathrm{Rb} 1 \mathrm{~B}^{\mathrm{viii}}-\mathrm{Ga} 1-\mathrm{Rb} 1 \mathrm{~A}^{\mathrm{xi}}$ & $122.12(14)$ \\
\hline $\mathrm{O} 3^{\mathrm{ii}}-\mathrm{Rb} 1 \mathrm{~A}-\mathrm{As} 2^{\mathrm{iii}}$ & $77.98(3)$ & $\mathrm{Rb} 1 \mathrm{~B}-\mathrm{Ga} 1-\mathrm{Rb} 1 \mathrm{~A}^{\mathrm{xi}}$ & $117.05(13)$ \\
\hline $\mathrm{O} 3^{\mathrm{v}}-\mathrm{Rb} 1 \mathrm{~A}-\mathrm{As} 2^{\mathrm{iii}}$ & $77.70(2)$ & $\mathrm{Rb} 1 \mathrm{~B}^{\mathrm{ix}}-\mathrm{Ga} 1-\mathrm{Rb} 1 \mathrm{~A}^{\mathrm{xi}}$ & $2.56(14)$ \\
\hline $\mathrm{O} 3^{\mathrm{i}}-\mathrm{Rb} 1 \mathrm{~A}-\mathrm{As} 2^{\mathrm{iii}}$ & $125.97(2)$ & $\mathrm{Rb} 1 \mathrm{~A}^{\mathrm{viii}}-\mathrm{Ga} 1-\mathrm{Rb} 1 \mathrm{~A}^{\mathrm{xi}}$ & $119.614(1)$ \\
\hline $\mathrm{O} 3-\mathrm{Rb} 1 \mathrm{~A}-\mathrm{As} 2^{\mathrm{iii}}$ & $134.60(2)$ & $\mathrm{Rb} 1 \mathrm{~A}-\mathrm{Ga} 1-\mathrm{Rb} 1 \mathrm{~A}^{\mathrm{xi}}$ & $119.614(1)$ \\
\hline $\mathrm{O} 2^{\mathrm{iv}}-\mathrm{Rb} 1 \mathrm{~A}-\mathrm{As} 2^{\mathrm{iii}}$ & $92.720(19)$ & $\mathrm{O} 1^{\mathrm{xii}}-\mathrm{As} 1-\mathrm{O} 1^{\mathrm{xiii}}$ & $92.59(5)$ \\
\hline $\mathrm{O} 2^{\mathrm{ii}}-\mathrm{Rb} 1 \mathrm{~A}-\mathrm{As} 2^{\mathrm{iii}}$ & $49.72(2)$ & $\mathrm{O} 1^{\mathrm{xii}}-\mathrm{As} 1-\mathrm{O} 1^{\mathrm{xiv}}$ & $92.59(5)$ \\
\hline $\mathrm{O} 2-\mathrm{Rb} 1 \mathrm{~A}-\mathrm{As} 2^{\mathrm{iii}}$ & $98.261(18)$ & $\mathrm{O} 1^{\mathrm{xiii}}-\mathrm{As} 1-\mathrm{O} 1^{\mathrm{xiv}}$ & $92.59(5)$ \\
\hline $\mathrm{O} 2^{\mathrm{v}}-\mathrm{Rb} 1 \mathrm{~A}-\mathrm{As} 2^{\mathrm{iii}}$ & $122.595(19)$ & $\mathrm{O} 1^{\mathrm{xii}}-\mathrm{As} 1-\mathrm{O} 1^{\mathrm{i}}$ & $87.41(5)$ \\
\hline 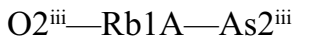 & $23.323(18)$ & $\mathrm{O} 1^{\mathrm{xiii}}-\mathrm{As} 1-\mathrm{O} 1^{\mathrm{i}}$ & $180.00(9)$ \\
\hline $\mathrm{O} 2^{\mathrm{i}}-\mathrm{Rb} 1 \mathrm{~A}-\mathrm{As} 2^{\mathrm{iii}}$ & $146.608(19)$ & $\mathrm{O} 1^{\mathrm{xiv}}-\mathrm{As} 1-\mathrm{O} 1^{\mathrm{i}}$ & $87.41(5)$ \\
\hline $\mathrm{As} 2^{\mathrm{iv}}-\mathrm{Rb} 1 \mathrm{~A}-\mathrm{As} 2^{\mathrm{iii}}$ & $99.334(9)$ & $\mathrm{O} 1^{\mathrm{xii}}-\mathrm{As} 1-\mathrm{O} 1^{\mathrm{ix}}$ & 180.0 \\
\hline $\mathrm{Rb} 1 \mathrm{~B}^{\mathrm{i}}-\mathrm{Rb} 1 \mathrm{~A}-\mathrm{As} 2^{\mathrm{v}}$ & $151.382(7)$ & $\mathrm{O} 1^{\mathrm{xiii}}-\mathrm{As} 1-\mathrm{O} 1^{\mathrm{ix}}$ & $87.41(5)$ \\
\hline $\mathrm{Rb} 1 \mathrm{~B}^{\mathrm{ii}}-\mathrm{Rb} 1 \mathrm{~A}-\mathrm{As} 2^{\mathrm{v}}$ & $67.49(6)$ & $\mathrm{O} 1^{\mathrm{xiv}}-\mathrm{As} 1-\mathrm{O} 1^{\mathrm{ix}}$ & $87.41(5)$ \\
\hline $\mathrm{O} 3^{\mathrm{iii}}-\mathrm{Rb} 1 \mathrm{~A}-\mathrm{As} 2^{\mathrm{v}}$ & $92.58(3)$ & $\mathrm{O} 1^{\mathrm{i}}-\mathrm{As} 1-\mathrm{O} 1^{\mathrm{ix}}$ & $92.58(5)$ \\
\hline $\mathrm{O} 3^{\mathrm{iv}}-\mathrm{Rb} 1 \mathrm{~A}-\mathrm{As} 2^{\mathrm{v}}$ & $77.70(2)$ & $\mathrm{O} 1^{\mathrm{xii}}-\mathrm{As} 1-\mathrm{O} 1^{\mathrm{viii}}$ & $87.42(5)$ \\
\hline 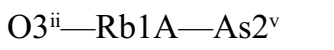 & $125.97(2)$ & 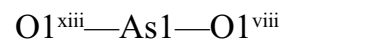 & $87.42(5)$ \\
\hline $\mathrm{O} 3^{\mathrm{v}}-\mathrm{Rb} 1 \mathrm{~A}-\mathrm{As} 2^{\mathrm{v}}$ & $24.04(2)$ & $\mathrm{O} 1^{\text {xiv }}-\mathrm{As} 1-\mathrm{O} 1^{\text {viii }}$ & $180.00(6)$ \\
\hline $\mathrm{O} 3^{\mathrm{i}}-\mathrm{Rb} 1 \mathrm{~A}-\mathrm{As} 2^{\mathrm{v}}$ & $134.60(2)$ & $\mathrm{O} 1^{\mathrm{i}}-\mathrm{As} 1-\mathrm{O} 1^{\mathrm{viii}}$ & $92.58(5)$ \\
\hline $\mathrm{O} 3-\mathrm{Rb} 1 \mathrm{~A}-\mathrm{As} 2^{\mathrm{v}}$ & $77.98(3)$ & $\mathrm{O} 1^{\mathrm{ix}}-\mathrm{As} 1-\mathrm{O} 1^{\text {viii }}$ & $92.58(5)$ \\
\hline $\mathrm{O} 2^{\mathrm{iv}}-\mathrm{Rb} 1 \mathrm{~A}-\mathrm{As} 2^{\mathrm{v}}$ & $122.596(19)$ & $\mathrm{O} 2-\mathrm{As} 2-\mathrm{O} 4^{\mathrm{iv}}$ & $117.31(6)$ \\
\hline 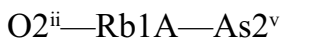 & $146.608(19)$ & $\mathrm{O} 2-\mathrm{As} 2-\mathrm{O} 1^{\mathrm{xv}}$ & $115.10(6)$ \\
\hline
\end{tabular}




\begin{tabular}{|c|c|c|c|}
\hline $\mathrm{O} 2-\mathrm{Rb} 1 \mathrm{~A}-\mathrm{As} 2^{\mathrm{v}}$ & $49.72(2)$ & $\mathrm{O} 4^{\mathrm{iv}}-\mathrm{As} 2-\mathrm{O}^{\mathrm{xv}}$ & $100.43(5)$ \\
\hline $\mathrm{O} 2^{\mathrm{v}}-\mathrm{Rb} 1 \mathrm{~A}-\mathrm{As} 2^{\mathrm{v}}$ & $23.323(17)$ & $\mathrm{O} 2-\mathrm{As} 2-\mathrm{O} 3$ & $104.11(6)$ \\
\hline $\mathrm{O} 2^{\mathrm{iii}}-\mathrm{Rb} 1 \mathrm{~A}-\mathrm{As} 2^{\mathrm{v}}$ & $92.72(2)$ & $\mathrm{O} 4^{\mathrm{iv}}-\mathrm{As} 2-\mathrm{O} 3$ & $111.03(6)$ \\
\hline $\mathrm{O} 2^{\mathrm{i}}-\mathrm{Rb} 1 \mathrm{~A}-\mathrm{As} 2^{\mathrm{v}}$ & $98.261(19)$ & $\mathrm{O} 1^{\mathrm{xv}}-\mathrm{As} 2-\mathrm{O} 3$ & $108.86(6)$ \\
\hline $\mathrm{As} 2^{\mathrm{iv}}-\mathrm{Rb} 1 \mathrm{~A}-\mathrm{As} 2^{\mathrm{v}}$ & $99.334(9)$ & $\mathrm{O} 2-\mathrm{As} 2-\mathrm{Rb} 1 \mathrm{~B}^{\mathrm{ii}}$ & $50.1(3)$ \\
\hline 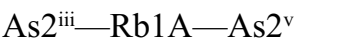 & $99.334(9)$ & $\mathrm{O} 4^{\mathrm{iv}}-\mathrm{As} 2-\mathrm{Rb} 1 \mathrm{~B}^{\mathrm{ii}}$ & $114.68(16)$ \\
\hline $\mathrm{Rb} 1 \mathrm{~B}-\mathrm{Rb} 1 \mathrm{~A}-\mathrm{As} 2^{\mathrm{ii}}$ & $60.329(3)$ & $\mathrm{O} 1^{\mathrm{xv}}-\mathrm{As} 2-\mathrm{Rb} 1 \mathrm{~B}^{\mathrm{ii}}$ & $144.86(16)$ \\
\hline $\mathrm{Rb}_{1 \mathrm{~B}}{ }^{\mathrm{ii}}-\mathrm{Rb} 1 \mathrm{~A}-\mathrm{As} 2^{\mathrm{ii}}$ & $67.49(6)$ & $\mathrm{O} 3-\mathrm{As} 2-\mathrm{Rb} 1 \mathrm{~B}^{\mathrm{ii}}$ & $58.0(2)$ \\
\hline $\mathrm{O} 3^{\mathrm{iii}}-\mathrm{Rb} 1 \mathrm{~A}-\mathrm{As} 2^{\mathrm{ii}}$ & $77.98(3)$ & $\mathrm{O} 2-\mathrm{As} 2-\mathrm{Rb} 1 \mathrm{~B}$ & $58.6(2)$ \\
\hline $\mathrm{O} 3^{\mathrm{iv}}-\mathrm{Rb} 1 \mathrm{~A}-\mathrm{As} 2^{\mathrm{ii}}$ & $134.60(2)$ & $\mathrm{O} 4^{\mathrm{iv}}-\mathrm{As} 2-\mathrm{Rb} 1 \mathrm{~B}$ & $106.7(3)$ \\
\hline $\mathrm{O} 3^{\mathrm{ii}}-\mathrm{Rb} 1 \mathrm{~A}-\mathrm{As} 2^{\mathrm{ii}}$ & $24.04(2)$ & $\mathrm{O} 1^{\mathrm{xv}}-\mathrm{As} 2-\mathrm{Rb} 1 \mathrm{~B}$ & $151.7(2)$ \\
\hline $\mathrm{O} 3^{\mathrm{v}}-\mathrm{Rb} 1 \mathrm{~A}-\mathrm{As} 2^{\mathrm{ii}}$ & $125.97(2)$ & $\mathrm{O} 3-\mathrm{As} 2-\mathrm{Rb} 1 \mathrm{~B}$ & $53.57(7)$ \\
\hline $\mathrm{O} 3^{\mathrm{i}}-\mathrm{Rb} 1 \mathrm{~A}-\mathrm{As} 2^{\mathrm{ii}}$ & $77.70(2)$ & $\mathrm{Rb} 1 \mathrm{~B}^{\mathrm{ii}}-\mathrm{As} 2-\mathrm{Rb} 1 \mathrm{~B}$ & $10.2(5)$ \\
\hline $\mathrm{O} 3-\mathrm{Rb} 1 \mathrm{~A}-\mathrm{As} 2^{\mathrm{ii}}$ & $92.58(2)$ & $\mathrm{O} 2-\mathrm{As} 2-\mathrm{Rb} 1 \mathrm{~A}$ & $54.94(4)$ \\
\hline $\mathrm{O} 2^{\mathrm{iv}}-\mathrm{Rb} 1 \mathrm{~A}-\mathrm{As} 2^{\mathrm{ii}}$ & $98.261(18)$ & $\mathrm{O} 4^{\mathrm{iv}}-\mathrm{As} 2-\mathrm{Rb} 1 \mathrm{~A}$ & $111.68(4)$ \\
\hline $\mathrm{O} 2^{\mathrm{ii}}-\mathrm{Rb} 1 \mathrm{~A}-\mathrm{As} 2^{\mathrm{ii}}$ & $23.324(18)$ & $\mathrm{O} 1^{\mathrm{xv}}-\mathrm{As} 2-\mathrm{Rb} 1 \mathrm{~A}$ & $147.34(4)$ \\
\hline $\mathrm{O} 2-\mathrm{Rb} 1 \mathrm{~A}-\mathrm{As} 2^{\mathrm{ii}}$ & $92.721(19)$ & $\mathrm{O} 3-\mathrm{As} 2-\mathrm{Rb} 1 \mathrm{~A}$ & $54.48(5)$ \\
\hline $\mathrm{O} 2^{\mathrm{v}}-\mathrm{Rb} 1 \mathrm{~A}-\mathrm{As} 2^{\mathrm{ii}}$ & $146.607(19)$ & $\mathrm{Rb} 1 \mathrm{~B}^{\mathrm{ii}}-\mathrm{As} 2-\mathrm{Rb} 1 \mathrm{~A}$ & $5.1(3)$ \\
\hline $\mathrm{O} 2^{\mathrm{iii}}-\mathrm{Rb} 1 \mathrm{~A}-\mathrm{As} 2^{\mathrm{ii}}$ & $49.72(2)$ & $\mathrm{Rb} 1 \mathrm{~B}-\mathrm{As} 2-\mathrm{Rb} 1 \mathrm{~A}$ & $5.4(3)$ \\
\hline $\mathrm{O} 2^{\mathrm{i}}-\mathrm{Rb} 1 \mathrm{~A}-\mathrm{As} 2^{\mathrm{ii}}$ & $122.596(19)$ & $\mathrm{O} 2-\mathrm{As} 2-\mathrm{Rb} 1 \mathrm{~B}^{\mathrm{i}}$ & $56.09(6)$ \\
\hline $\mathrm{As} 2^{\mathrm{iv}}-\mathrm{Rb} 1 \mathrm{~A}-\mathrm{As} 2^{\mathrm{ii}}$ & $120.658(6)$ & $\mathrm{O} 4^{\mathrm{iv}}-\mathrm{As} 2-\mathrm{Rb} 1 \mathrm{~B}^{\mathrm{i}}$ & $113.41(9)$ \\
\hline $\mathrm{As} 2^{\mathrm{iii}}-\mathrm{Rb} 1 \mathrm{~A}-\mathrm{As} 2^{\mathrm{ii}}$ & $57.236(12)$ & $\mathrm{O} 1^{\mathrm{xv}}-\mathrm{As} 2-\mathrm{Rb} 1 \mathrm{~B}^{\mathrm{i}}$ & $145.26(10)$ \\
\hline $\mathrm{As} 2^{\mathrm{v}}-\mathrm{Rb} 1 \mathrm{~A}-\mathrm{As} 2^{\mathrm{ii}}$ & $134.983(5)$ & $\mathrm{O} 3-\mathrm{As} 2-\mathrm{Rb} 1 \mathrm{~B}^{\mathrm{i}}$ & $52.42(10)$ \\
\hline $\mathrm{Rb} 1 \mathrm{~B}-\mathrm{Rb} 1 \mathrm{~B}-\mathrm{Rb} 1 \mathrm{~B}^{\mathrm{ii}}$ & $60.00(2)$ & $\mathrm{Rb} 1 \mathrm{~B}^{\mathrm{ii}}-\mathrm{As} 2-\mathrm{Rb}_{1 \mathrm{~B}}{ }^{\mathrm{i}}$ & $6.1(3)$ \\
\hline $\mathrm{Rb} 1 \mathrm{~B}^{\mathrm{i}}-\mathrm{Rb} 1 \mathrm{~B}-\mathrm{O} 2^{\mathrm{v}}$ & $161.6(2)$ & $\mathrm{Rb} 1 \mathrm{~B}-\mathrm{As} 2-\mathrm{Rb} 1 \mathrm{~B}^{\mathrm{i}}$ & $6.7(3)$ \\
\hline $\mathrm{Rb} 1 \mathrm{~B}^{\mathrm{ii}}-\mathrm{Rb} 1 \mathrm{~B}-\mathrm{O} 2^{\mathrm{v}}$ & $108.4(3)$ & $\mathrm{Rb} 1 \mathrm{~A}-\mathrm{As} 2-\mathrm{Rb} 1 \mathrm{~B}^{\mathrm{i}}$ & $2.49(11)$ \\
\hline $\mathrm{Rb} 1 \mathrm{~B}-\mathrm{Rb} 1 \mathrm{~B}-\mathrm{O} 2^{\mathrm{i}}$ & $108.4(2)$ & $\mathrm{O} 2-\mathrm{As} 2-\mathrm{Rb} 1 \mathrm{~B}^{\mathrm{xvi}}$ & $93.36(7)$ \\
\hline $\mathrm{Rb} 1 \mathrm{~B}^{\mathrm{ii}}-\mathrm{Rb} 1 \mathrm{~B}-\mathrm{O} 2^{\mathrm{i}}$ & $161.60(17)$ & $\mathrm{O} 4^{\mathrm{iv}}-\mathrm{As} 2-\mathrm{Rb} 1 \mathrm{~B}^{\mathrm{xvi}}$ & $42.23(4)$ \\
\hline $\mathrm{O} 2^{\mathrm{v}}-\mathrm{Rb} 1 \mathrm{~B}-\mathrm{O} 2^{\mathrm{i}}$ & $86.3(5)$ & $\mathrm{O} 1^{\mathrm{xv}}-\mathrm{As} 2-\mathrm{Rb} 1 \mathrm{~B}^{\mathrm{xvi}}$ & $80.78(10)$ \\
\hline $\mathrm{Rb} 1 \mathrm{~B}-\mathrm{Rb} 1 \mathrm{~B}-\mathrm{O} 3^{\mathrm{iv}}$ & $91.2(3)$ & $\mathrm{O} 3-\mathrm{As} 2-\mathrm{Rb} 1 \mathrm{~B}^{\mathrm{xvi}}$ & $153.23(5)$ \\
\hline $\mathrm{Rb} 1 \mathrm{~B}^{\mathrm{ii}}-\mathrm{Rb} 1 \mathrm{~B}-\mathrm{O}^{\mathrm{iv}}$ & $122.4(3)$ & $\mathrm{Rb} 1 \mathrm{~B}^{\mathrm{ii}}-\mathrm{As} 2-\mathrm{Rb} 1 \mathrm{~B}^{\mathrm{xvi}}$ & $126.58(16)$ \\
\hline $\mathrm{O} 2^{\mathrm{v}}-\mathrm{Rb} 1 \mathrm{~B}-\mathrm{O} 3^{\mathrm{iv}}$ & $83.6(3)$ & $\mathrm{Rb} 1 \mathrm{~B}-\mathrm{As} 2-\mathrm{Rb} 1 \mathrm{~B}^{\mathrm{xvi}}$ & $125.4(2)$ \\
\hline 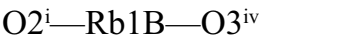 & $69.1(3)$ & $\mathrm{Rb} 1 \mathrm{~A}-\mathrm{As} 2-\mathrm{Rb} \mathrm{B}^{\mathrm{xvi}}$ & $127.56(9)$ \\
\hline $\mathrm{Rb} 1 \mathrm{~B}-\mathrm{Rb} 1 \mathrm{~B}-\mathrm{O} 3$ & $122.4(3)$ & $\mathrm{Rb} 1 \mathrm{~B}-\mathrm{As} 2-\mathrm{Rb} 1 \mathrm{~B}^{\mathrm{xvi}}$ & $130.05(2)$ \\
\hline $\mathrm{Rb} 1 \mathrm{~B}^{\mathrm{ii}}-\mathrm{Rb} 1 \mathrm{~B}-\mathrm{O} 3$ & $91.2(3)$ & $\mathrm{O} 2-\mathrm{As} 2-\mathrm{Rb} 1 \mathrm{~A}^{\mathrm{xvii}}$ & $94.58(4)$ \\
\hline $\mathrm{O} 2 \mathrm{v}-\mathrm{Rb} 1 \mathrm{~B}-\mathrm{O} 3$ & $69.1(3)$ & $\mathrm{O} 4^{\mathrm{iv}}-\mathrm{As} 2-\mathrm{Rb} 1 \mathrm{~A}^{\mathrm{xvii}}$ & $42.53(4)$ \\
\hline $\mathrm{O} 2 \mathrm{i}-\mathrm{Rb} 1 \mathrm{~B}-\mathrm{O} 3$ & $83.6(3)$ & $\mathrm{O} 1^{\mathrm{xv}}-\mathrm{As} 2-\mathrm{Rb} 1 \mathrm{~A}^{\mathrm{xvii}}$ & $79.08(4)$ \\
\hline $\mathrm{O} 3^{\mathrm{iv}}-\mathrm{Rb} 1 \mathrm{~B}-\mathrm{O} 3$ & $142.5(7)$ & $\mathrm{O} 3-\mathrm{As} 2-\mathrm{Rb} 1 \mathrm{~A}^{\mathrm{xvii}}$ & $153.36(5)$ \\
\hline $\mathrm{Rb} 1 \mathrm{~B}^{\mathrm{i}}-\mathrm{Rb} 1 \mathrm{~B}-\mathrm{O} 3^{\mathrm{v}}$ & $113.4(3)$ & $\mathrm{Rb} 1 \mathrm{~B}^{\mathrm{ii}}-\mathrm{As} 2-\mathrm{Rb} 1 \mathrm{~A}^{\mathrm{xvii}}$ & $128.36(6)$ \\
\hline $\mathrm{Rb} 1 \mathrm{~B}^{\mathrm{ii}}-\mathrm{Rb} 1 \mathrm{~B}-\mathrm{O}^{\mathrm{v}}$ & $76.7(3)$ & $\mathrm{Rb} 1 \mathrm{~B}-\mathrm{As} 2-\mathrm{Rb} 1 \mathrm{~A}^{\mathrm{xvii}}$ & $127.17(12)$ \\
\hline $\mathrm{O} 2^{\mathrm{v}}-\mathrm{Rb} 1 \mathrm{~B}-\mathrm{O} 3^{\mathrm{v}}$ & $48.28(11)$ & $\mathrm{Rb} 1 \mathrm{~A}-\mathrm{As} 2-\mathrm{Rb} 1 \mathrm{~A}^{\mathrm{xvii}}$ & $129.347(10)$ \\
\hline $\mathrm{O} 2^{\mathrm{i}}-\mathrm{Rb} 1 \mathrm{~B}-\mathrm{O}^{\mathrm{v}}$ & $121.7(5)$ & $\mathrm{Rb} 1 \mathrm{~B}^{\mathrm{i}}-\mathrm{As} 2-\mathrm{Rb} 1 \mathrm{~A}^{\mathrm{xvii}}$ & $131.84(12)$ \\
\hline $\mathrm{O}^{\mathrm{iv}}-\mathrm{Rb} 1 \mathrm{~B}-\mathrm{O}^{\mathrm{v}}$ & $71.12(11)$ & $\mathrm{Rb}_{1} \mathrm{~B}^{\mathrm{xvi}}-\mathrm{As} 2-\mathrm{Rb} 1 \mathrm{~A}^{\mathrm{xvii}}$ & $1.79(10)$ \\
\hline $\mathrm{O} 3-\mathrm{Rb} 1 \mathrm{~B}-\mathrm{O}^{\mathrm{v}}$ & $105.18(19)$ & $\mathrm{O} 2-\mathrm{As} 2-\mathrm{Rb} 1 \mathrm{~B}^{\mathrm{xviii}}$ & $92.74(11)$ \\
\hline $\mathrm{Rb} 1 \mathrm{~B}^{\mathrm{i}}-\mathrm{Rb} 1 \mathrm{~B}-\mathrm{O}^{\mathrm{i}}$ & $76.7(3)$ & $\mathrm{O} 4^{\mathrm{iv}}-\mathrm{As} 2-\mathrm{Rb}_{1} \mathrm{~B}^{\mathrm{xviii}}$ & $46.4(2)$ \\
\hline $\mathrm{Rb}_{1 \mathrm{~B}} \mathrm{ii}-\mathrm{Rb} 1 \mathrm{~B}-\mathrm{O}^{\mathrm{i}}$ & $113.4(3)$ & $\mathrm{O} 1^{\mathrm{xv}}-\mathrm{As} 2-\mathrm{Rb} 1 \mathrm{~B}^{\mathrm{xviii}}$ & $76.75(13)$ \\
\hline $\mathrm{O} 2^{\mathrm{v}}-\mathrm{Rb} 1 \mathrm{~B}-\mathrm{O} 3^{\mathrm{i}}$ & $121.7(5)$ & $\mathrm{O} 3-\mathrm{As} 2-\mathrm{Rb} 1 \mathrm{~B}^{\mathrm{xviii}}$ & $157.05(19)$ \\
\hline 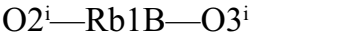 & $48.28(11)$ & $\mathrm{Rb} 1 \mathrm{~B}^{\mathrm{ii}}-\mathrm{As} 2-\mathrm{Rb} 1 \mathrm{~B}^{\mathrm{xviii}}$ & $129.10(5)$ \\
\hline
\end{tabular}




\begin{tabular}{|c|c|}
\hline $\mathrm{O}^{3}{ }^{\mathrm{iv}}-\mathrm{Rb} 1 \mathrm{~B}-\mathrm{O}^{\mathrm{i}}$ & $105.18(19)$ \\
\hline $\mathrm{O} 3-\mathrm{Rb} 1 \mathrm{~B}-\mathrm{O}^{\mathrm{i}}$ & $71.12(11)$ \\
\hline $\mathrm{O}^{\mathrm{v}}-\mathrm{Rb} 1 \mathrm{~B}-\mathrm{O}^{\mathrm{i}}$ & $169.0(7)$ \\
\hline $\mathrm{Rb} 1 \mathrm{~B}-\mathrm{Rb} 1 \mathrm{~B}-\mathrm{O} 2$ & $118.6(3)$ \\
\hline $\mathrm{Rb} 1 \mathrm{~B}^{\mathrm{ii}}-\mathrm{Rb} 1 \mathrm{~B}-\mathrm{O} 2$ & $60.4(4)$ \\
\hline $\mathrm{O} 2^{\mathrm{v}}-\mathrm{Rb} 1 \mathrm{~B}-\mathrm{O} 2$ & $56.66(12)$ \\
\hline 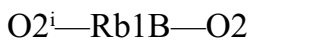 & $124.3(5)$ \\
\hline $\mathrm{O}{ }^{\mathrm{iv}}-\mathrm{Rb} 1 \mathrm{~B}-\mathrm{O} 2$ & $133.6(2)$ \\
\hline $\mathrm{O} 3-\mathrm{Rb} 1 \mathrm{~B}-\mathrm{O} 2$ & $46.84(5)$ \\
\hline $\mathrm{O}^{\mathrm{v}}-\mathrm{Rb} 1 \mathrm{~B}-\mathrm{O} 2$ & $64.78(4)$ \\
\hline 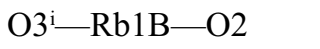 & $115.33(5)$ \\
\hline $\mathrm{Rb} 1 \mathrm{~B}-\mathrm{Rb} 1 \mathrm{~B}-\mathrm{O} 2^{\mathrm{iv}}$ & $60.4(3)$ \\
\hline $\mathrm{Rb} 1 \mathrm{~B}^{\mathrm{ii}}-\mathrm{Rb} 1 \mathrm{~B}-\mathrm{O} 2^{\mathrm{iv}}$ & $118.6(3)$ \\
\hline $\mathrm{O} 2^{\mathrm{v}}-\mathrm{Rb} 1 \mathrm{~B}-\mathrm{O} 2^{\mathrm{iv}}$ & $124.3(5)$ \\
\hline $\mathrm{O} 2^{\mathrm{i}}-\mathrm{Rb} 1 \mathrm{~B}-\mathrm{O} 2^{\mathrm{iv}}$ & $56.66(12)$ \\
\hline $\mathrm{O} 3^{\text {iv }}-\mathrm{Rb} 1 \mathrm{~B}-\mathrm{O} 2^{\mathrm{iv}}$ & $46.84(5)$ \\
\hline $\mathrm{O} 3-\mathrm{Rb} 1 \mathrm{~B}-\mathrm{O} 2^{\mathrm{iv}}$ & $133.6(2)$ \\
\hline $\mathrm{O} 3^{\mathrm{v}}-\mathrm{Rb} 1 \mathrm{~B}-\mathrm{O} 2^{\mathrm{iv}}$ & $115.33(5)$ \\
\hline $\mathrm{O} 3^{\mathrm{i}}-\mathrm{Rb} 1 \mathrm{~B}-\mathrm{O} 2^{\mathrm{iv}}$ & $64.78(4)$ \\
\hline $\mathrm{O} 2-\mathrm{Rb} 1 \mathrm{~B}-\mathrm{O} 2^{\mathrm{iv}}$ & $179.0(7)$ \\
\hline $\mathrm{Rb} 1 \mathrm{~B}-\mathrm{Rb} 1 \mathrm{~B}-\mathrm{O} 3^{\mathrm{iii}}$ & $48.36(16)$ \\
\hline $\mathrm{Rb} 1 \mathrm{~B}^{\mathrm{ii}}-\mathrm{Rb} 1 \mathrm{~B}-\mathrm{O} 3^{\mathrm{iii}}$ & $56.58(15)$ \\
\hline $\mathrm{O} 2^{\mathrm{v}}-\mathrm{Rb} 1 \mathrm{~B}-\mathrm{O} 3^{\mathrm{iii}}$ & $113.81(3)$ \\
\hline $\mathrm{O} 2^{\mathrm{i}}-\mathrm{Rb} 1 \mathrm{~B}-\mathrm{O} 3^{\mathrm{iii}}$ & $128.32(3)$ \\
\hline $\mathrm{O}^{\mathrm{iv}}-\mathrm{Rb} 1 \mathrm{~B}-\mathrm{O}^{\mathrm{iii}}$ & $66.90(12)$ \\
\hline $\mathrm{O} 3-\mathrm{Rb} 1 \mathrm{~B}-\mathrm{O} 3^{\mathrm{iii}}$ & $147.5(4)$ \\
\hline $\mathrm{O} 3^{\mathrm{v}}-\mathrm{Rb} 1 \mathrm{~B}-\mathrm{O} 3^{\mathrm{iii}}$ & $66.07(15)$ \\
\hline $\mathrm{O} 3^{\mathrm{i}}-\mathrm{Rb} 1 \mathrm{~B}-\mathrm{O} 3^{\mathrm{iii}}$ & $122.7(4)$ \\
\hline $\mathrm{O} 2-\mathrm{Rb} 1 \mathrm{~B}-\mathrm{O} 3^{\mathrm{iii}}$ & $105.6(3)$ \\
\hline $\mathrm{O} 2^{\mathrm{iv}}-\mathrm{Rb} 1 \mathrm{~B}-\mathrm{O} 3^{\mathrm{iii}}$ & $73.63(18)$ \\
\hline $\mathrm{Rb} 1 \mathrm{~B}^{\mathrm{i}}-\mathrm{Rb} 1 \mathrm{~B}-\mathrm{O} 3^{\mathrm{ii}}$ & $56.58(18)$ \\
\hline $\mathrm{Rb} 1 \mathrm{~B}^{\mathrm{ii}}-\mathrm{Rb} 1 \mathrm{~B}-\mathrm{O} 3^{\mathrm{ii}}$ & $48.36(15)$ \\
\hline $\mathrm{O} 2^{\mathrm{v}}-\mathrm{Rb} 1 \mathrm{~B}-\mathrm{O}^{3 \mathrm{ii}}$ & $128.32(3)$ \\
\hline $\mathrm{O} 2^{\mathrm{i}}-\mathrm{Rb} 1 \mathrm{~B}-\mathrm{O} 3^{\mathrm{ii}}$ & $113.81(3)$ \\
\hline $\mathrm{O}^{\mathrm{iv}}-\mathrm{Rb} 1 \mathrm{~B}-\mathrm{O} 3^{\mathrm{ii}}$ & $147.5(4)$ \\
\hline $\mathrm{O} 3-\mathrm{Rb} 1 \mathrm{~B}-\mathrm{O} 3^{\mathrm{ii}}$ & $66.90(12)$ \\
\hline $\mathrm{O}^{2}-\mathrm{Rb} 1 \mathrm{~B}-\mathrm{O}^{\mathrm{ii}}$ & $122.7(4)$ \\
\hline $\mathrm{O} 3^{\mathrm{i}}-\mathrm{Rb} 1 \mathrm{~B}-\mathrm{O} 3^{\mathrm{ii}}$ & $66.07(15)$ \\
\hline $\mathrm{O} 2-\mathrm{Rb} 1 \mathrm{~B}-\mathrm{O} 3^{\mathrm{ii}}$ & $73.63(18)$ \\
\hline $\mathrm{O} 2^{\mathrm{iv}}-\mathrm{Rb} 1 \mathrm{~B}-\mathrm{O} 3^{\mathrm{ii}}$ & $105.6(3)$ \\
\hline 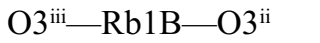 & $90.9(4)$ \\
\hline $\mathrm{Rb} 1 \mathrm{~B}-\mathrm{Rb} 1 \mathrm{~B}-\mathrm{O} 2^{\mathrm{ii}}$ & $15.05(2)$ \\
\hline $\mathrm{Rb} 1 \mathrm{~B}^{\mathrm{ii}}-\mathrm{Rb} 1 \mathrm{~B}-\mathrm{O} 2^{\mathrm{ii}}$ & $52.10(7)$ \\
\hline $\mathrm{O} 2^{\mathrm{v}}-\mathrm{Rb} 1 \mathrm{~B}-\mathrm{O} 2^{\mathrm{ii}}$ & $160.5(4)$ \\
\hline $\mathrm{O} 2^{\mathrm{i}}-\mathrm{Rb} 1 \mathrm{~B}-\mathrm{O} 2^{\mathrm{ii}}$ & $112.87(12)$ \\
\hline $\mathrm{O} 3^{\mathrm{iv}}-\mathrm{Rb} 1 \mathrm{~B}-\mathrm{O} 2^{\mathrm{ii}}$ & $106.2(3)$ \\
\hline $\mathrm{O} 3-\mathrm{Rb} 1 \mathrm{~B}-\mathrm{O} 2^{\mathrm{ii}}$ & $107.8(3)$ \\
\hline $\mathrm{O} 3^{\mathrm{v}}-\mathrm{Rb} 1 \mathrm{~B}-\mathrm{O} 2^{\mathrm{ii}}$ & $118.1(4)$ \\
\hline
\end{tabular}

\begin{tabular}{|c|c|}
\hline $\mathrm{Rb} 1 \mathrm{~B}-\mathrm{As} 2-\mathrm{Rb} 1 \mathrm{~B}^{\mathrm{xviii}}$ & $128.75(3)$ \\
\hline $\mathrm{Rb} 1 \mathrm{~A}-\mathrm{As} 2-\mathrm{Rb} 1 \mathrm{~B}^{\mathrm{xviii}}$ & $130.52(5)$ \\
\hline $\mathrm{Rb} 1 \mathrm{~B}^{\mathrm{i}}-\mathrm{As} 2-\mathrm{Rb} 1 \mathrm{~B}^{\mathrm{xviii}}$ & $132.99(17)$ \\
\hline $\mathrm{Rb} 1 \mathrm{~B}^{\mathrm{xvi}}-\mathrm{As} 2-\mathrm{Rb} 1 \mathrm{~B}^{\mathrm{xviii}}$ & $4.8(2)$ \\
\hline Rb1A ${ }^{x v i i}-A s 2-R b 1 B^{x v i i i}$ & $3.88(18)$ \\
\hline $\mathrm{O} 2-\mathrm{As} 2-\mathrm{Rb} 1 \mathrm{~B}^{\mathrm{xvii}}$ & $97.48(15)$ \\
\hline $\mathrm{O} 4^{\mathrm{iv}}-\mathrm{As} 2-\mathrm{Rb} 1 \mathrm{~B}^{\mathrm{xvii}}$ & $39.02(18)$ \\
\hline $\mathrm{O} 1^{\mathrm{xv}}-\mathrm{As} 2-\mathrm{Rb} 1 \mathrm{~B}^{\mathrm{xvii}}$ & $79.91(7)$ \\
\hline $\mathrm{O} 3-\mathrm{As} 2-\mathrm{Rb} 1 \mathrm{~B}^{\mathrm{xvii}}$ & $149.72(18)$ \\
\hline $\mathrm{Rb} 1 \mathrm{~B}^{\mathrm{ii}}-\mathrm{As} 2-\mathrm{Rb} 1 \mathrm{~B}^{\mathrm{xvii}}$ & $129.012(18$ \\
\hline Rb1B-As2-Rb1B ${ }^{\mathrm{xvii}}$ & $127.02(15)$ \\
\hline $\mathrm{Rb} 1 \mathrm{~A}-\mathrm{As} 2-\mathrm{Rb} 1 \mathrm{~B}^{\mathrm{xvii}}$ & $129.587(13)$ \\
\hline $\mathrm{Rb} \mathrm{B}^{\mathrm{i}}-\mathrm{As} 2-\mathrm{Rb}^{\mathrm{x}} \mathrm{Bvii}$ & $132.07(12)$ \\
\hline $\mathrm{Rb} 1 \mathrm{~B}^{\mathrm{xvi}}-\mathrm{As} 2-\mathrm{Rb} 1 \mathrm{~B}^{\mathrm{xvii}}$ & $4.2(2)$ \\
\hline $\mathrm{Rb} 1 \mathrm{~A}^{\mathrm{xvii}}-\mathrm{As} 2-\mathrm{Rb} 1 \mathrm{~B}^{\mathrm{xvii}}$ & $3.66(17)$ \\
\hline Rb1B ${ }^{x v i i i}-A s 2-R b 1 B^{x v i i}$ & $7.4(3)$ \\
\hline $\mathrm{As} 2^{\mathrm{xix}}-\mathrm{O} 1-\mathrm{As} 1^{\mathrm{xx}}$ & $131.96(6)$ \\
\hline As $2^{\text {xix }}-\mathrm{O} 1-\mathrm{Rb}_{1} \mathrm{~B}^{\mathrm{vii}}$ & $79.11(18)$ \\
\hline $\mathrm{As} 1^{\mathrm{xx}}-\mathrm{O} 1-\mathrm{Rb} 1 \mathrm{~B}^{\mathrm{vii}}$ & $129.23(10)$ \\
\hline As $2-\mathrm{O} 2-\mathrm{Ga}^{\mathrm{xvii}}$ & $123.99(6)$ \\
\hline $\mathrm{As} 2-\mathrm{O} 2-\mathrm{Rb}_{1} \mathrm{~B}^{\mathrm{ii}}$ & $105.9(2)$ \\
\hline $\mathrm{Ga} 1^{\mathrm{xvi}}-\mathrm{O} 2-\mathrm{Rb}_{1} \mathrm{~B}^{\mathrm{ii}}$ & $125.54(18)$ \\
\hline As2-O2-Rb1B & $96.8(3)$ \\
\hline $\mathrm{Ga}^{\mathrm{xvii}}-\mathrm{O} 2-\mathrm{Rb} 1 \mathrm{~B}$ & $130.47(10)$ \\
\hline $\mathrm{Rb} \mathrm{B}^{\mathrm{ii}}-\mathrm{O} 2-\mathrm{Rb} 1 \mathrm{~B}$ & $11.2(6)$ \\
\hline $\mathrm{As} 2-\mathrm{O} 2-\mathrm{Rb} 1 \mathrm{~A}$ & $101.74(4)$ \\
\hline $\mathrm{Ga} 1^{\mathrm{xvii}}-\mathrm{O} 2-\mathrm{Rb} 1 \mathrm{~A}$ & $128.51(4)$ \\
\hline $\mathrm{Rb} 1 \mathrm{~B}^{\mathrm{ii}}-\mathrm{O} 2-\mathrm{Rb} 1 \mathrm{~A}$ & $4.6(2)$ \\
\hline $\mathrm{Rb} 1 \mathrm{~B}-\mathrm{O} 2-\mathrm{Rb} 1 \mathrm{~A}$ & $6.8(3)$ \\
\hline $\mathrm{As} 2-\mathrm{O} 2-\mathrm{Rb}_{1} \mathrm{~B}^{\mathrm{i}}$ & $102.64(6)$ \\
\hline $\mathrm{Ga}^{\mathrm{xvii}}-\mathrm{O} 2-\mathrm{Rb} \mathrm{B}^{\mathrm{i}}$ & $128.80(4)$ \\
\hline $\mathrm{Rb} 1 \mathrm{~B}^{\mathrm{ii}}-\mathrm{O} 2-\mathrm{Rb} \mathrm{B}^{\mathrm{i}}$ & $3.34(18)$ \\
\hline $\mathrm{Rb} 1 \mathrm{~B}-\mathrm{O} 2-\mathrm{Rb}^{\mathrm{i}} \mathrm{B}^{\mathrm{i}}$ & $9.3(4)$ \\
\hline $\mathrm{Rb} 1 \mathrm{~A}-\mathrm{O} 2-\mathrm{Rb}^{\mathrm{B}} \mathrm{B}$ & $2.77(12)$ \\
\hline As2-O3-Rb1B & $101.51(6)$ \\
\hline As2-O3-Rb1B ${ }^{\mathrm{ii}}$ & $96.3(3)$ \\
\hline $\mathrm{Rb} 1 \mathrm{~B}-\mathrm{O} 3-\mathrm{Rb} 1 \mathrm{~B}^{\mathrm{ii}}$ & $12.1(6)$ \\
\hline As2-O3-Rb1A & $101.48(6)$ \\
\hline $\mathrm{Rb} 1 \mathrm{~B}-\mathrm{O} 3-\mathrm{Rb} 1 \mathrm{~A}$ & $6.5(3)$ \\
\hline $\mathrm{Rb} 1 \mathrm{~B}^{\mathrm{ii}}-\mathrm{O} 3-\mathrm{Rb} 1 \mathrm{~A}$ & $6.8(3)$ \\
\hline As2-O3-Rb1B ${ }^{\mathrm{i}}$ & $106.0(2)$ \\
\hline $\mathrm{Rb} 1 \mathrm{~B}-\mathrm{O} 3-\mathrm{Rb} \mathrm{B}^{\mathrm{i}}$ & $9.3(5)$ \\
\hline $\mathrm{Rb} 1 \mathrm{~B}^{\mathrm{ii}}-\mathrm{O} 3-\mathrm{Rb} \mathrm{B}^{\mathrm{i}}$ & $10.1(5)$ \\
\hline $\mathrm{Rb} 1 \mathrm{~A}-\mathrm{O} 3-\mathrm{Rb} 1 \mathrm{~B}^{\mathrm{i}}$ & $4.9(2)$ \\
\hline $\mathrm{As} 2^{\mathrm{iv}}-\mathrm{O} 4-\mathrm{Ga}^{\mathrm{xx}}$ & $126.28(6)$ \\
\hline As $2^{i v}-O 4-\mathrm{Rb}_{1} \mathrm{~B}^{\mathrm{xxi}}$ & $120.71(10)$ \\
\hline $\mathrm{Ga} 1^{\mathrm{xx}}-\mathrm{O} 4-\mathrm{Rb}_{1} \mathrm{~B}^{\mathrm{xxi}}$ & $99.25(5)$ \\
\hline As $2^{\text {iv }}-\mathrm{O} 4-\mathrm{Rb} 1 \mathrm{~A}^{\mathrm{xix}}$ & $121.85(5)$ \\
\hline
\end{tabular}




\begin{tabular}{|c|c|}
\hline $\mathrm{O} 3^{\mathrm{i}}-\mathrm{Rb} 1 \mathrm{~B}-\mathrm{O} 2^{\mathrm{ii}}$ & $72.8(2)$ \\
\hline $\mathrm{O} 2-\mathrm{Rb} 1 \mathrm{~B}-\mathrm{O} 2^{\mathrm{ii}}$ & $106.5(4)$ \\
\hline $\mathrm{O} 2^{\mathrm{iv}}-\mathrm{Rb} 1 \mathrm{~B}-\mathrm{O} 2^{\mathrm{ii}}$ & $72.5(2)$ \\
\hline $\mathrm{O} 3^{\mathrm{iii}}-\mathrm{Rb} 1 \mathrm{~B}-\mathrm{O} 2^{\mathrm{ii}}$ & $57.9(3)$ \\
\hline 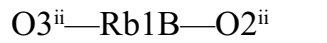 & 41.55 (19) \\
\hline $\mathrm{Rb} 1 \mathrm{~B}^{\mathrm{i}}-\mathrm{Rb} 1 \mathrm{~B}-\mathrm{O} 2^{\mathrm{iii}}$ & $52.10(12)$ \\
\hline $\mathrm{Rb} 1 \mathrm{~B}^{\mathrm{ii}}-\mathrm{Rb} 1 \mathrm{~B}-\mathrm{O} 2^{\mathrm{iii}}$ & $15.05(5)$ \\
\hline $\mathrm{O} 2^{\mathrm{v}}-\mathrm{Rb} 1 \mathrm{~B}-\mathrm{O} 2^{\mathrm{iii}}$ & $112.87(12)$ \\
\hline $\mathrm{O} 2^{\mathrm{i}}-\mathrm{Rb} 1 \mathrm{~B}-\mathrm{O} 2^{\mathrm{iii}}$ & $160.5(4)$ \\
\hline $\mathrm{O} 3^{\mathrm{iv}}-\mathrm{Rb} 1 \mathrm{~B}-\mathrm{O} 2^{\mathrm{iii}}$ & $107.8(3)$ \\
\hline $\mathrm{O} 3-\mathrm{Rb} 1 \mathrm{~B}-\mathrm{O} 2^{\mathrm{iii}}$ & $106.2(3)$ \\
\hline $\mathrm{O} 3^{\mathrm{v}}-\mathrm{Rb} 1 \mathrm{~B}-\mathrm{O} 2^{\mathrm{iii}}$ & $72.8(2)$ \\
\hline 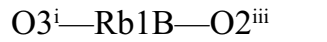 & $118.1(4)$ \\
\hline $\mathrm{O} 2-\mathrm{Rb} 1 \mathrm{~B}-\mathrm{O} 2^{\mathrm{iii}}$ & $72.5(2)$ \\
\hline $\mathrm{O} 2^{\mathrm{iv}}-\mathrm{Rb} 1 \mathrm{~B}-\mathrm{O} 2^{\mathrm{iii}}$ & $106.5(4)$ \\
\hline 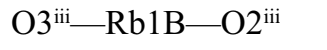 & $41.55(19)$ \\
\hline $\mathrm{O} 3^{i \mathrm{ii}}-\mathrm{Rb} 1 \mathrm{~B}-\mathrm{O} 2^{\mathrm{iii}}$ & $57.9(3)$ \\
\hline $\mathrm{O} 2^{\mathrm{ii}}-\mathrm{Rb} 1 \mathrm{~B}-\mathrm{O} 2^{\mathrm{iii}}$ & $48.4(2)$ \\
\hline $\mathrm{Rb} 1 \mathrm{~B}^{\mathrm{i}}-\mathrm{Rb} 1 \mathrm{~B}-\mathrm{O} 4^{\mathrm{vi}}$ & $153.31(5)$ \\
\hline $\mathrm{Rb} 1 \mathrm{~B}^{\mathrm{ii}}-\mathrm{Rb} 1 \mathrm{~B}-\mathrm{O}^{\mathrm{vi}}$ & $130.76(15)$ \\
\hline $\mathrm{O} 2^{\mathrm{v}}-\mathrm{Rb} 1 \mathrm{~B}-\mathrm{O} 4^{\mathrm{vi}}$ & $45.1(2)$ \\
\hline $\mathrm{O} 2^{\mathrm{i}}-\mathrm{Rb} 1 \mathrm{~B}-\mathrm{O} 4^{\mathrm{vi}}$ & $53.4(3)$ \\
\hline $\mathrm{O} 3^{\mathrm{iv}}-\mathrm{Rb} 1 \mathrm{~B}-\mathrm{O} 4^{\mathrm{vi}}$ & $98.0(5)$ \\
\hline $\mathrm{O} 3-\mathrm{Rb} 1 \mathrm{~B}-\mathrm{O} 4^{\mathrm{vi}}$ & $44.50(19)$ \\
\hline $\mathrm{O} 3^{\mathrm{v}}-\mathrm{Rb} 1 \mathrm{~B}-\mathrm{O} 4^{\mathrm{vi}}$ & $93.3(3)$ \\
\hline $\mathrm{O} 3^{\mathrm{i}}-\mathrm{Rb} 1 \mathrm{~B}-\mathrm{O} 4^{\mathrm{vi}}$ & $76.7(3)$ \\
\hline $\mathrm{O} 2-\mathrm{Rb} 1 \mathrm{~B}-\mathrm{O} 4^{\mathrm{vi}}$ & $71.6(2)$ \\
\hline $\mathrm{O} 2^{\mathrm{iv}}-\mathrm{Rb} 1 \mathrm{~B}-\mathrm{O} 4^{\mathrm{vi}}$ & $109.4(4)$ \\
\hline 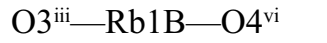 & $157.2(3)$ \\
\hline $\mathrm{O} 3^{\mathrm{ii}}-\mathrm{Rb} 1 \mathrm{~B}-\mathrm{O} 4^{\mathrm{vi}}$ & $109.34(9)$ \\
\hline $\mathrm{O} 2^{\mathrm{ii}}-\mathrm{Rb} 1 \mathrm{~B}-\mathrm{O} 4^{\mathrm{vi}}$ & $144.95(3)$ \\
\hline $\mathrm{O} 2^{\mathrm{iii}}-\mathrm{Rb} 1 \mathrm{~B}-\mathrm{O} 4^{\mathrm{vi}}$ & $144.11(3)$ \\
\hline 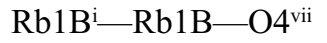 & $130.76(11)$ \\
\hline
\end{tabular}

$\mathrm{Ga} 1^{\mathrm{xx}}-\mathrm{O} 4-\mathrm{Rb}_{1} \mathrm{~A}^{\mathrm{xix}}$

$\mathrm{Rb}^{\mathrm{Bxi}}-\mathrm{O} 4-\mathrm{Rb}^{\mathrm{x}} \mathrm{A}^{\mathrm{xix}}$

As2 $2^{\text {iv }}-\mathrm{O} 4-\mathrm{Rb}_{1} \mathrm{~B}^{\mathrm{xix}}$

$\mathrm{Ga} 1^{\mathrm{xx}}-\mathrm{O} 4-\mathrm{Rb}^{\mathrm{B}}{ }^{\mathrm{xix}}$

$\mathrm{Rb}^{\mathrm{Bxi}}-\mathrm{O} 4-\mathrm{Rb}^{\mathrm{x}} \mathrm{B}^{\mathrm{xix}}$

$\mathrm{Rb} \mathrm{A}^{\mathrm{xix}}-\mathrm{O} 4-\mathrm{Rb}^{\mathrm{x}} \mathrm{B}^{\mathrm{xix}}$

As $2^{\mathrm{iv}}-\mathrm{O} 4-\mathrm{Rb} 1 \mathrm{~B}^{\mathrm{xxii}}$

$\mathrm{Ga}^{\mathrm{xx}}-\mathrm{O} 4-\mathrm{Rb}^{\mathrm{x}} \mathrm{B}^{\mathrm{xxi}}$

$\mathrm{Rb}^{\mathrm{x}}{ }^{\mathrm{xxi}}-\mathrm{O} 4-\mathrm{Rb}^{\mathrm{B}} \mathrm{B}^{\mathrm{xxi}}$

$\mathrm{Rb} 1 \mathrm{~A}^{\mathrm{xix}}-\mathrm{O} 4-\mathrm{Rb}_{1} \mathrm{~B}^{\mathrm{xxii}}$

$\mathrm{Rb}^{\mathrm{B}} \mathrm{B}^{\mathrm{xix}}-\mathrm{O} 4-\mathrm{Rb}^{\mathrm{B}} \mathrm{B}^{\mathrm{xxii}}$

As2 $2^{\mathrm{iv}}$ - O4- Rb1B

$\mathrm{Ga}^{\mathrm{xx}}-\mathrm{O} 4-\mathrm{Rb} 1 \mathrm{~B}$

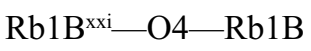

$\mathrm{Rb} 1 A^{\mathrm{xix}}-\mathrm{O} 4-\mathrm{Rb} 1 \mathrm{~B}$

$\mathrm{Rb}^{\mathrm{B}} \mathrm{B}^{\mathrm{xix}}-\mathrm{O} 4-\mathrm{Rb} 1 \mathrm{~B}$

$\mathrm{Rb}^{\mathrm{B} \times \mathrm{xii}}-\mathrm{O} 4-\mathrm{Rb} 1 \mathrm{~B}$

$\mathrm{As} 2^{\mathrm{iv}}-\mathrm{O} 4-\mathrm{Rb}^{\mathrm{B}} \mathrm{B}^{\mathrm{i}}$

$\mathrm{Ga}^{\mathrm{xx}}-\mathrm{O} 4-\mathrm{Rb} \mathrm{B}^{\mathrm{i}}$

$\mathrm{Rb}^{\mathrm{xxi}}-\mathrm{O} 4-\mathrm{Rb}^{\mathrm{x}} \mathrm{B}^{\mathrm{i}}$

$\mathrm{Rb}^{\mathrm{A}} \mathrm{A}^{\mathrm{xix}}-\mathrm{O} 4-\mathrm{Rb}^{\mathrm{B}} \mathrm{B}^{\mathrm{i}}$

$\mathrm{Rb}^{\mathrm{x}}{ }^{\mathrm{xix}}-\mathrm{O} 4-\mathrm{Rb}^{\mathrm{B}} \mathrm{B}^{\mathrm{i}}$

$\mathrm{Rb}_{13}{ }^{\mathrm{xxi}}-\mathrm{O} 4-\mathrm{Rb}_{1} \mathrm{~B}^{\mathrm{i}}$

$\mathrm{Rb} 1 \mathrm{~B}-\mathrm{O} 4-\mathrm{Rb}^{\mathrm{B}} \mathrm{B}^{\mathrm{i}}$

As $2^{\mathrm{i}}-\mathrm{O} 4-\mathrm{Rb} 1 \mathrm{~A}$

$\mathrm{Ga}^{\mathrm{xx}}-\mathrm{O} 4-\mathrm{Rb} 1 \mathrm{~A}$

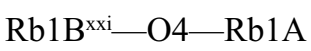

$\mathrm{Rb} 1 A^{\mathrm{xix}}-\mathrm{O} 4-\mathrm{Rb} 1 \mathrm{~A}$

$\mathrm{Rb}^{\mathrm{x}}{ }^{\mathrm{xix}}-\mathrm{O} 4-\mathrm{Rb} 1 \mathrm{~A}$

$\mathrm{Rb}^{\mathrm{x} x \mathrm{xii}}-\mathrm{O} 4-\mathrm{Rb} 1 \mathrm{~A}$

$\mathrm{Rb} 1 \mathrm{~B}-\mathrm{O} 4-\mathrm{Rb} 1 \mathrm{~A}$

$\mathrm{Rb} 1 \mathrm{~B}^{\mathrm{i}}-\mathrm{O} 4-\mathrm{Rb} 1 \mathrm{~A}$
$99.67(4)$

$2.51(13)$

$126.9(3)$

96.29 (16)

7.1 (3)

5.2 (2)

117.91 (18)

103.25 (18)

4.1 (2)

3.99 (18)

9.0 (4)

53.63 (19)

72.96 (19)

$134.20(8)$

136.70 (6)

139.53 (16)

135.79 (4)

47.20 (15)

80.09 (19)

130.0 (3)

132.53 (17)

136.12 (3)

131.0 (3)

8.2 (4)

50.21 (3)

76.56 (3)

133.03 (14)

135.53 (3)

$138.77(15)$

134.28 (7)

3.8 (2)

4.5 (2)

Symmetry codes: (i) $-y, x-y, z$; (ii) $-x+y,-x, z$; (iii) $x-y,-y,-z+3 / 2$; (iv) $-x,-x+y,-z+3 / 2$; (v) $y, x,-z+3 / 2$; (vi) $y, x-1,-z+3 / 2$; (vii) $-y, x-y-1, z$; (viii) $x$, $y+1, z$; (ix) $-x+y+1,-x+1, z$; (x) $-y, x-y+1, z$; (xi) $x+1, y+1, z$; (xii) $x-y-1 / 3, x+1 / 3,-z+4 / 3$; (xiii) $y+2 / 3,-x+y+4 / 3,-z+4 / 3$; (xiv) $-x+2 / 3,-y+1 / 3,-z+4 / 3$; (xv) $x-1, y, z$; (xvi) $-y-1, x-y-1, z$; (xvii) $x-1, y-1, z$; (xviii) $-x+y-1,-x-1, z$; (xix) $x+1, y, z$; (xx) $x, y-1, z$; (xxi) $-x+y+1,-x, z$; (xxii) $-y+1, x-y, z$.

Hydrogen-bond geometry $\left(\AA,{ }^{\circ}\right)$

\begin{tabular}{lllll}
\hline$D-\mathrm{H} \cdots A$ & $D-\mathrm{H}$ & $\mathrm{H} \cdots A$ & $D \cdots A$ & $D-\mathrm{H} \cdots A$ \\
\hline $\mathrm{O} 3-\mathrm{H}^{\cdots} \mathrm{O}^{\mathrm{vi}}$ & $0.80(3)$ & $1.98(3)$ & $2.7314(17)$ & $158(3)$ \\
\hline
\end{tabular}

Symmetry code: (vi) $y, x-1,-z+3 / 2$.

Rubidium gallium bis[hydrogen arsenate(V)] (RbGaHAsO42)

Crystal data

$\mathrm{RbGa}\left(\mathrm{HAsO}_{4}\right)_{2}$

Trigonal, $R \overline{3} c: H$

$M_{r}=435.05$

$a=8.385(1) \AA$ 
$c=53.880(11) \AA$

$V=3280.7(10) \AA^{3}$

$Z=18$

$F(000)=3600$

$D_{\mathrm{x}}=3.964 \mathrm{Mg} \mathrm{m}^{-3}$

Mo $K \alpha$ radiation, $\lambda=0.71073 \AA$

Data collection

Nonius KappaCCD single-crystal four-circle diffractometer

Radiation source: fine-focus sealed tube

$\varphi$ and $\omega$ scans

Absorption correction: multi-scan

(SCALEPACK; Otwinowski et al., 2003)

$T_{\min }=0.343, T_{\max }=0.697$

3896 measured reflections

\section{Refinement}

Refinement on $F^{2}$

Least-squares matrix: full

$R\left[F^{2}>2 \sigma\left(F^{2}\right)\right]=0.016$

$w R\left(F^{2}\right)=0.040$

$S=1.11$

1079 reflections

68 parameters

2 restraints

Primary atom site location: structure-invariant direct methods

Secondary atom site location: difference Fourier map

\section{Special details}

Geometry. All esds (except the esd in the dihedral angle between two 1.s. planes) are estimated using the full covariance matrix. The cell esds are taken into account individually in the estimation of esds in distances, angles and torsion angles; correlations between esds in cell parameters are only used when they are defined by crystal symmetry. An approximate (isotropic) treatment of cell esds is used for estimating esds involving 1.s. planes.

Fractional atomic coordinates and isotropic or equivalent isotropic displacement parameters $\left(\AA^{2}\right)$

\begin{tabular}{llllll}
\hline & $x$ & $y$ & $z$ & $U_{\text {iso }} * U_{\text {eq }}$ & Occ. $(<1)$ \\
\hline Rb1A & 0.000000 & 0.000000 & 0.750000 & $0.0297(12)$ & $0.909(3)$ \\
Rb1B & 0.000000 & $-0.050(5)$ & 0.750000 & $0.011(3)$ & $0.0304(9)$ \\
$\mathrm{Rb} 2$ & 0.000000 & 0.000000 & $0.66714(2)$ & $0.02912(12)$ & \\
$\mathrm{Ga} 1$ & 0.333333 & 0.666667 & $0.75374(2)$ & $0.00733(9)$ & \\
$\mathrm{Ga} 2$ & 0.333333 & 0.666667 & 0.666667 & $0.00817(11)$ & \\
$\mathrm{As}$ & $-0.43059(3)$ & $-0.39514(3)$ & $0.71280(2)$ & $0.00799(7)$ & \\
$\mathrm{O} 1$ & $0.4536(2)$ & $-0.4402(2)$ & $0.68636(3)$ & $0.0159(3)$ & \\
$\mathrm{O} 2$ & $-0.4459(2)$ & $-0.2541(2)$ & $0.73338(3)$ & $0.0106(3)$ & \\
$\mathrm{O} 3$ & $-0.1974(2)$ & $-0.2813(2)$ & $0.70523(3)$ & $0.0178(3)$ & \\
$\mathrm{O} 4$ & $0.4789(2)$ & $-0.1223(2)$ & $0.77582(3)$ & $0.0103(3)$ & \\
$\mathrm{H}$ & $-0.161(5)$ & $-0.354(5)$ & $0.7099(6)$ & $0.035(10) *$ & \\
& & & & &
\end{tabular}

Cell parameters from 2663 reflections

$\theta=2.3-30.0^{\circ}$

$\mu=19.42 \mathrm{~mm}^{-1}$

$T=293 \mathrm{~K}$

Hexagonal plate, colourless

$0.07 \times 0.07 \times 0.02 \mathrm{~mm}$

1079 independent reflections

1027 reflections with $I>2 \sigma(I)$

$R_{\text {int }}=0.016$

$\theta_{\text {max }}=30.0^{\circ}, \theta_{\min }=2.3^{\circ}$

$h=-11 \rightarrow 11$

$k=-9 \rightarrow 9$

$l=-75 \rightarrow 75$

Hydrogen site location: difference Fourier map

All H-atom parameters refined

$w=1 /\left[\sigma^{2}\left(F_{\mathrm{o}}^{2}\right)+(0.0168 P)^{2}+20.8962 P\right]$

where $P=\left(F_{\mathrm{o}}^{2}+2 F_{\mathrm{c}}^{2}\right) / 3$

$(\Delta / \sigma)_{\max }=0.014$

$\Delta \rho_{\max }=0.79 \mathrm{e} \AA^{-3}$

$\Delta \rho_{\min }=-0.52$ e $\AA^{-3}$

Extinction correction: SHELXL2016

(Sheldrick, 2015),

$\mathrm{Fc}^{*}=\mathrm{kFc}\left[1+0.001 \mathrm{xFc}^{2} \lambda^{3} / \sin (2 \theta)\right]^{-1 / 4}$

Extinction coefficient: 0.000092 (13) 
Atomic displacement parameters $\left(\AA^{2}\right)$

\begin{tabular}{lllllll}
\hline & $U^{11}$ & $U^{22}$ & $U^{33}$ & $U^{12}$ & $U^{13}$ & $U^{23}$ \\
\hline $\mathrm{Rb} 1 \mathrm{~A}$ & $0.0333(16)$ & $0.0333(16)$ & $0.0225(9)$ & $0.0166(8)$ & 0.000 & 0.000 \\
$\mathrm{Rb} 1 \mathrm{~B}$ & $0.023(11)$ & $0.012(7)$ & $0.004(7)$ & $0.011(5)$ & $-0.002(4)$ & $-0.001(2)$ \\
$\mathrm{Rb} 2$ & $0.03481(17)$ & $0.03481(17)$ & $0.0177(2)$ & $0.01740(9)$ & 0.000 & 0.000 \\
$\mathrm{Ga} 1$ & $0.00785(12)$ & $0.00785(12)$ & $0.00629(16)$ & $0.00393(6)$ & 0.000 & 0.000 \\
$\mathrm{Ga} 2$ & $0.00943(15)$ & $0.00943(15)$ & $0.0057(2)$ & $0.00472(8)$ & 0.000 & 0.000 \\
$\mathrm{As}$ & $0.00991(11)$ & $0.00839(10)$ & $0.00729(10)$ & $0.00580(8)$ & $0.00068(7)$ & $0.00083(7)$ \\
$\mathrm{O} 1$ & $0.0246(8)$ & $0.0212(8)$ & $0.0087(6)$ & $0.0164(7)$ & $-0.0050(6)$ & $-0.0011(6)$ \\
$\mathrm{O} 2$ & $0.0105(7)$ & $0.0106(6)$ & $0.0104(6)$ & $0.0049(5)$ & $0.0026(5)$ & $-0.0016(5)$ \\
$\mathrm{O} 3$ & $0.0129(7)$ & $0.0173(8)$ & $0.0257(9)$ & $0.0093(7)$ & $0.0085(6)$ & $0.0093(6)$ \\
$\mathrm{O} 4$ & $0.0116(6)$ & $0.0092(6)$ & $0.0120(6)$ & $0.0066(6)$ & $-0.0019(5)$ & $-0.0038(5)$ \\
\hline
\end{tabular}

Geometric parameters $\left(\stackrel{A}{\circ}{ }^{\circ}\right)$

\begin{tabular}{|c|c|c|c|}
\hline $\mathrm{Rb} 1 \mathrm{~A}-\mathrm{Rb} 1 \mathrm{~B}^{\mathrm{i}}$ & $0.42(4)$ & $\mathrm{Rb} 2-\mathrm{O} 3^{\mathrm{ii}}$ & $2.9347(17)$ \\
\hline $\mathrm{Rb} 1 \mathrm{~A}-\mathrm{Rb} 1 \mathrm{~B}^{\mathrm{ii}}$ & $0.42(4)$ & $\mathrm{Rb} 2-\mathrm{O} 1^{\mathrm{vi}}$ & $3.3714(16)$ \\
\hline $\mathrm{Rb} 1 \mathrm{~A}-\mathrm{O} 3^{\mathrm{iii}}$ & $3.197(2)$ & $\mathrm{Rb} 2-\mathrm{O} 1^{\mathrm{vii}}$ & $3.3715(16)$ \\
\hline $\mathrm{Rb} 1 \mathrm{~A}-\mathrm{O}^{\mathrm{iv}}$ & $3.197(2)$ & $\mathrm{Rb} 2-\mathrm{O} 1^{\text {viii }}$ & $3.3715(17)$ \\
\hline $\mathrm{Rb} 1 \mathrm{~A}-\mathrm{O} 3^{\mathrm{ii}}$ & $3.197(2)$ & $\mathrm{Rb} 2-\mathrm{O} 4^{\mathrm{ix}}$ & $3.4960(16)$ \\
\hline $\mathrm{Rb} 1 \mathrm{~A}-\mathrm{O} 3^{\mathrm{i}}$ & $3.197(2)$ & $\mathrm{Rb} 2-\mathrm{O} 4^{\mathrm{x}}$ & $3.4960(17)$ \\
\hline $\mathrm{Rb} 1 \mathrm{~A}-\mathrm{O}^{\mathrm{v}}$ & $3.197(2)$ & $\mathrm{Rb} 2-\mathrm{O} 4^{\mathrm{xi}}$ & $3.4960(16)$ \\
\hline $\mathrm{Rb} 1 \mathrm{~A}-\mathrm{O} 3$ & $3.197(2)$ & $\mathrm{Rb} 2-\mathrm{O} 3^{\mathrm{xii}}$ & 3.5327 (19) \\
\hline $\mathrm{Rb} 1 \mathrm{~A}-\mathrm{O} 2^{\mathrm{iv}}$ & $3.3698(16)$ & $\mathrm{Rb} 2-\mathrm{O} 3^{\text {xiii }}$ & $3.533(2)$ \\
\hline $\mathrm{Rb} 1 \mathrm{~A}-\mathrm{O} 2^{\mathrm{ii}}$ & $3.3698(16)$ & $\mathrm{Rb} 2-\mathrm{O} 3^{\mathrm{xiv}}$ & $3.5328(19)$ \\
\hline $\mathrm{Rb} 1 \mathrm{~A}-\mathrm{O} 2^{\mathrm{v}}$ & $3.3699(16)$ & $\mathrm{Rb} 2-\mathrm{As}^{\mathrm{xiv}}$ & $3.7432(5)$ \\
\hline $\mathrm{Rb} 1 \mathrm{~A}-\mathrm{O} 2$ & $3.3699(16)$ & $\mathrm{Rb} 2-\mathrm{As}^{\mathrm{xii}}$ & $3.7432(5)$ \\
\hline $\mathrm{Rb} 1 \mathrm{~A}-\mathrm{O} 2^{\mathrm{iii}}$ & $3.3699(15)$ & $\mathrm{Rb} 2-\mathrm{As}^{\mathrm{xiii}}$ & $3.7432(5)$ \\
\hline $\mathrm{Rb} 1 \mathrm{~A}-\mathrm{O} 2^{\mathrm{i}}$ & $3.3699(15)$ & $\mathrm{Ga} 1-\mathrm{O} 2^{\mathrm{xv}}$ & $1.9596(14)$ \\
\hline $\mathrm{Rb} 1 \mathrm{~A}-\mathrm{As}^{\mathrm{iv}}$ & $4.0083(5)$ & $\mathrm{Ga} 1-\mathrm{O} 2^{\mathrm{ii}}$ & $1.9597(15)$ \\
\hline $\mathrm{Rb} 1 \mathrm{~B}-\mathrm{Rb} 1 \mathrm{~B}^{\mathrm{i}}$ & $0.72(7)$ & $\mathrm{Ga} 1-\mathrm{O} 2^{\mathrm{xvi}}$ & $1.9597(15)$ \\
\hline $\mathrm{Rb} 1 \mathrm{~B}-\mathrm{Rb} 1 \mathrm{~B}^{\mathrm{ii}}$ & $0.72(7)$ & $\mathrm{Ga} 1-\mathrm{O} 4^{\mathrm{xvii}}$ & $1.9690(15)$ \\
\hline $\mathrm{Rb} 1 \mathrm{~B}-\mathrm{O} 3^{\text {iv }}$ & $3.019(15)$ & $\mathrm{Ga} 1-\mathrm{O} 4^{\mathrm{i}}$ & $1.9690(15)$ \\
\hline $\mathrm{Rb} 1 \mathrm{~B}-\mathrm{O} 3$ & $3.019(15)$ & $\mathrm{Ga} 1-\mathrm{O} 4^{\text {xiii }}$ & $1.9690(15)$ \\
\hline $\mathrm{Rb} 1 \mathrm{~B}-\mathrm{O} 2^{\mathrm{v}}$ & $3.05(3)$ & $\mathrm{Ga} 2-\mathrm{O} 1^{\text {viii }}$ & $1.9625(15)$ \\
\hline $\mathrm{Rb} 1 \mathrm{~B}-\mathrm{O} 2^{\mathrm{i}}$ & $3.05(3)$ & $\mathrm{Ga} 2-\mathrm{O} 1^{\mathrm{xiv}}$ & $1.9625(16)$ \\
\hline $\mathrm{Rb} 1 \mathrm{~B}-\mathrm{O} 3^{\mathrm{i}}$ & $3.161(2)$ & $\mathrm{Ga} 2-\mathrm{O} 1^{\mathrm{xix}}$ & $1.9625(15)$ \\
\hline $\mathrm{Rb} 1 \mathrm{~B}-\mathrm{O}^{\mathrm{v}}$ & $3.161(2)$ & $\mathrm{Ga} 2-\mathrm{O} 1^{\mathrm{i}}$ & $1.9626(15)$ \\
\hline $\mathrm{Rb} 1 \mathrm{~B}-\mathrm{O} 2^{\mathrm{iv}}$ & $3.363(2)$ & $\mathrm{Ga} 2-\mathrm{O} 1^{\text {xviii }}$ & $1.9626(16)$ \\
\hline $\mathrm{Rb} 1 \mathrm{~B}-\mathrm{O} 2$ & $3.363(2)$ & $\mathrm{Ga} 2-\mathrm{O}^{\mathrm{xvii}}$ & $1.9626(15)$ \\
\hline $\mathrm{Rb} 1 \mathrm{~B}-\mathrm{O}^{\mathrm{iii}}$ & 3.47 (3) & $\mathrm{As}-\mathrm{O} 1^{\mathrm{xx}}$ & $1.6576(15)$ \\
\hline $\mathrm{Rb} 1 \mathrm{~B}-\mathrm{O}^{\mathrm{ii}}$ & 3.47 (3) & $\mathrm{As}-\mathrm{O} 2$ & $1.6724(15)$ \\
\hline $\mathrm{Rb} 2-\mathrm{O} 3$ & $2.9346(17)$ & $\mathrm{As}-\mathrm{O} 4^{\mathrm{iv}}$ & $1.6805(15)$ \\
\hline $\mathrm{Rb} 2-\mathrm{O}^{\mathrm{i}}$ & 2.9347 (17) & $\mathrm{As}-\mathrm{O} 3$ & 1.7417 (17) \\
\hline $\mathrm{Rb} 1 \mathrm{~B}^{\mathrm{i}}-\mathrm{Rb} 1 \mathrm{~A}-\mathrm{Rb} 1 \mathrm{~B}^{\mathrm{ii}}$ & $120.00(15)$ & $\mathrm{O} 1^{\mathrm{vi}}-\mathrm{Rb} 2-\mathrm{As}^{\mathrm{xi}}$ & $26.29(3)$ \\
\hline $\mathrm{Rb} 1 \mathrm{~B}^{\mathrm{i}}-\mathrm{Rb} 1 \mathrm{~A}-\mathrm{O} 3^{\mathrm{iii}}$ & $61.38(3)$ & $\mathrm{O} 1^{\mathrm{vii}}-\mathrm{Rb} 2-\mathrm{As}^{\mathrm{xii}}$ & $88.53(3)$ \\
\hline
\end{tabular}




\begin{tabular}{|c|c|c|c|}
\hline $\mathrm{Rb} 1 \mathrm{~B}^{\mathrm{ii}}-\mathrm{Rb} 1 \mathrm{~A}-\mathrm{O} 3^{\mathrm{iii}}$ & $81.43(11)$ & $\mathrm{O} 1^{\mathrm{vii}}-\mathrm{Rb} 2-\mathrm{As}^{\mathrm{xii}}$ & $105.88(3)$ \\
\hline $\mathrm{Rb} 1 \mathrm{~B}^{\mathrm{i}}-\mathrm{Rb} 1 \mathrm{~A}-\mathrm{O} 3^{\mathrm{iv}}$ & $81.43(3)$ & $\mathrm{O} 4^{\mathrm{ix}}-\mathrm{Rb} 2-\mathrm{As}^{\mathrm{xii}}$ & $26.56(2)$ \\
\hline $\mathrm{Rb} 1 \mathrm{~B}^{\mathrm{ii}}-\mathrm{Rb} 1 \mathrm{~A}-\mathrm{O} 3^{\mathrm{iv}}$ & $128.90(5)$ & $\mathrm{O} 4^{\mathrm{x}}-\mathrm{Rb} 2-\mathrm{As}^{\mathrm{xii}}$ & $54.15(3)$ \\
\hline $\mathrm{O} 3^{\mathrm{iii}}-\mathrm{Rb} 1 \mathrm{~A}-\mathrm{O}^{\mathrm{iv}}$ & $69.26(5)$ & $\mathrm{O} 4^{\mathrm{xi}}-\mathrm{Rb} 2-\mathrm{As}^{\mathrm{xii}}$ & $71.43(3)$ \\
\hline $\mathrm{Rb} 1 \mathrm{~B}^{\mathrm{i}}-\mathrm{Rb} 1 \mathrm{~A}-\mathrm{O} 3^{\mathrm{ii}}$ & $81.43(3)$ & $\mathrm{O} 3^{\mathrm{xii}}-\mathrm{Rb} 2-\mathrm{As}^{\mathrm{xii}}$ & $27.50(3)$ \\
\hline $\mathrm{Rb} 1 \mathrm{~B}^{\mathrm{ii}}-\mathrm{Rb} 1 \mathrm{~A}-\mathrm{O} 3^{\mathrm{ii}}$ & $61.38(9)$ & $\mathrm{O} 3^{\mathrm{xiii}}-\mathrm{Rb} 2-\mathrm{As}^{\mathrm{xii}}$ & $63.12(3)$ \\
\hline 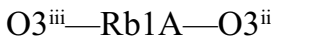 & $102.21(6)$ & $\mathrm{O} 3^{\mathrm{xiv}}-\mathrm{Rb} 2-\mathrm{As}^{\mathrm{xii}}$ & $98.11(3)$ \\
\hline $\mathrm{O} 3^{\mathrm{iv}}-\mathrm{Rb} 1 \mathrm{~A}-\mathrm{O} 3^{\mathrm{ii}}$ & $162.87(7)$ & $\mathrm{As} s^{\mathrm{xiv}}-\mathrm{Rb} 2-\mathrm{As}^{\mathrm{xii}}$ & $79.914(13)$ \\
\hline $\mathrm{Rb} 1 \mathrm{~B}^{\mathrm{i}}-\mathrm{Rb} 1 \mathrm{~A}-\mathrm{O} 3^{\mathrm{i}}$ & $61.38(3)$ & $\mathrm{O} 3-\mathrm{Rb} 2-\mathrm{As}^{\mathrm{xiii}}$ & $177.27(4)$ \\
\hline $\mathrm{Rb} 1 \mathrm{~B}^{\mathrm{ii}}-\mathrm{Rb} 1 \mathrm{~A}-\mathrm{O} 3^{\mathrm{i}}$ & $128.90(5)$ & $\mathrm{O} 3^{\mathrm{i}}-\mathrm{Rb} 2-\mathrm{As}^{\mathrm{xii}}$ & $100.79(4)$ \\
\hline $\mathrm{O} 3^{\mathrm{iii}}-\mathrm{Rb} 1 \mathrm{~A}-\mathrm{O} 3^{\mathrm{i}}$ & $122.76(7)$ & $\mathrm{O} 3^{\mathrm{ii}}-\mathrm{Rb} 2-\mathrm{As}^{\mathrm{xiii}}$ & $102.80(4)$ \\
\hline $\mathrm{O}^{\mathrm{iv}}-\mathrm{Rb} 1 \mathrm{~A}-\mathrm{O} 3^{\mathrm{i}}$ & $102.21(6)$ & $\mathrm{O} 1^{\mathrm{vi}}-\mathrm{Rb} 2-\mathrm{As}^{\mathrm{xiii}}$ & $88.53(3)$ \\
\hline $\mathrm{O} 3^{\mathrm{ii}}-\mathrm{Rb} 1 \mathrm{~A}-\mathrm{O} 3^{\mathrm{i}}$ & $69.26(5)$ & $\mathrm{O} 1^{\mathrm{vii}}-\mathrm{Rb} 2-\mathrm{As}^{\mathrm{xiii}}$ & $105.88(3)$ \\
\hline $\mathrm{Rb} 1 \mathrm{~B}^{\mathrm{i}}-\mathrm{Rb} 1 \mathrm{~A}-\mathrm{O} 3^{\mathrm{v}}$ & $128.90(3)$ & $\mathrm{O} 1^{\text {viii }}-\mathrm{Rb} 2-\mathrm{As}^{\mathrm{xiii}}$ & $26.29(3)$ \\
\hline $\mathrm{Rb} 1 \mathrm{~B}^{\mathrm{ii}}-\mathrm{Rb} 1 \mathrm{~A}-\mathrm{O}^{\mathrm{v}}$ & $61.38(9)$ & $\mathrm{O} 4^{\mathrm{ix}}-\mathrm{Rb} 2-\mathrm{As}^{\mathrm{xiii}}$ & $54.15(3)$ \\
\hline $\mathrm{O} 3^{\mathrm{iii}}-\mathrm{Rb} 1 \mathrm{~A}-\mathrm{O} 3^{\mathrm{v}}$ & $69.26(5)$ & $\mathrm{O} 4^{\mathrm{x}}-\mathrm{Rb} 2-\mathrm{As}^{\mathrm{xiii}}$ & $71.43(3)$ \\
\hline $\mathrm{O} 3^{\mathrm{iv}}-\mathrm{Rb} 1 \mathrm{~A}-\mathrm{O} 3^{\mathrm{v}}$ & $69.26(5)$ & $\mathrm{O} 4^{\mathrm{xi}}-\mathrm{Rb} 2-\mathrm{As}^{\mathrm{xiii}}$ & $26.56(3)$ \\
\hline $\mathrm{O} 3^{\mathrm{ii}}-\mathrm{Rb} 1 \mathrm{~A}-\mathrm{O}^{\mathrm{v}}$ & $122.76(6)$ & $\mathrm{O} 3^{\mathrm{xii}}-\mathrm{Rb} 2-\mathrm{As}^{\mathrm{xii}}$ & $98.11(3)$ \\
\hline $\mathrm{O} 3^{\mathrm{i}}-\mathrm{Rb} 1 \mathrm{~A}-\mathrm{O}^{\mathrm{v}}$ & $162.87(6)$ & $\mathrm{O} 3^{\mathrm{xii}}-\mathrm{Rb} 2-\mathrm{As}^{\mathrm{xiii}}$ & $27.50(3)$ \\
\hline $\mathrm{Rb} 1 \mathrm{~B}^{\mathrm{i}}-\mathrm{Rb} 1 \mathrm{~A}-\mathrm{O} 3$ & $128.90(3)$ & $\mathrm{O} 3^{\text {xiv }}-\mathrm{Rb} 2-\mathrm{As}^{\mathrm{xiii}}$ & $63.12(3)$ \\
\hline $\mathrm{Rb} 1 \mathrm{~B}^{\mathrm{ii}}-\mathrm{Rb} 1 \mathrm{~A}-\mathrm{O} 3$ & $81.43(11)$ & $\mathrm{As}^{\mathrm{xiv}}-\mathrm{Rb} 2-\mathrm{As}^{\mathrm{xiii}}$ & $79.914(13)$ \\
\hline 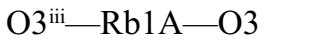 & $162.87(6)$ & $A s^{x i i}-R b 2-A s^{x i i i}$ & $79.914(13)$ \\
\hline $\mathrm{O} 3^{\mathrm{iv}}-\mathrm{Rb} 1 \mathrm{~A}-\mathrm{O} 3$ & $122.76(6)$ & $\mathrm{O} 2^{\mathrm{xv}}-\mathrm{Ga} 1-\mathrm{O} 2^{\mathrm{ii}}$ & $91.72(6)$ \\
\hline 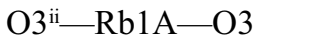 & $69.26(5)$ & $\mathrm{O} 2^{\mathrm{xv}}-\mathrm{Ga} 1-\mathrm{O} 2^{\mathrm{xvi}}$ & $91.72(6)$ \\
\hline $\mathrm{O} 3^{\mathrm{i}}-\mathrm{Rb} 1 \mathrm{~A}-\mathrm{O} 3$ & $69.26(5)$ & $\mathrm{O} 2^{\mathrm{ii}}-\mathrm{Ga} 1-\mathrm{O} 2^{\mathrm{xvi}}$ & $91.72(6)$ \\
\hline $\mathrm{O} 3^{\mathrm{v}}-\mathrm{Rb} 1 \mathrm{~A}-\mathrm{O} 3$ & $102.21(6)$ & $\mathrm{O} 2^{\mathrm{xv}}-\mathrm{Ga} 1-\mathrm{O} 4^{\mathrm{xvii}}$ & $92.25(6)$ \\
\hline $\mathrm{Rb} 1 \mathrm{~B}-\mathrm{Rb} 1 \mathrm{~A}-\mathrm{O} 2^{\mathrm{iv}}$ & $37.49(3)$ & $\mathrm{O} 2^{\mathrm{ii}}-\mathrm{Ga} 1-\mathrm{O} 4^{\mathrm{xvii}}$ & $175.98(6)$ \\
\hline $\mathrm{Rb} 1 \mathrm{~B}^{\mathrm{ii}}-\mathrm{Rb} 1 \mathrm{~A}-\mathrm{O} 2^{\mathrm{iv}}$ & $150.57(13)$ & $\mathrm{O} 2^{\mathrm{xvi}}-\mathrm{Ga} 1-\mathrm{O} 4^{\mathrm{xvii}}$ & $88.72(6)$ \\
\hline $\mathrm{O} 3^{\mathrm{iii}}-\mathrm{Rb} 1 \mathrm{~A}-\mathrm{O} 2^{\mathrm{iv}}$ & $70.43(4)$ & $\mathrm{O} 2^{\mathrm{xv}}-\mathrm{Ga} 1-\mathrm{O} 4^{\mathrm{i}}$ & $88.72(6)$ \\
\hline $\mathrm{O} 3^{\mathrm{iv}}-\mathrm{Rb} 1 \mathrm{~A}-\mathrm{O} 2^{\mathrm{iv}}$ & $48.04(4)$ & $\mathrm{O} 2^{\mathrm{ii}}-\mathrm{Ga} 1-\mathrm{O} 4^{\mathrm{i}}$ & $92.25(6)$ \\
\hline 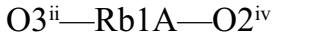 & $115.61(4)$ & $\mathrm{O} 2^{\mathrm{xvi}}-\mathrm{Ga} 1-\mathrm{O} 4^{\mathrm{i}}$ & $175.98(7)$ \\
\hline $\mathrm{O} 3^{\mathrm{i}}-\mathrm{Rb} 1 \mathrm{~A}-\mathrm{O} 2^{\mathrm{iv}}$ & $64.84(4)$ & $\mathrm{O} 4^{\mathrm{xvii}}-\mathrm{Ga} 1-\mathrm{O} 4^{\mathrm{i}}$ & $87.28(6)$ \\
\hline $\mathrm{O} 3^{\mathrm{v}}-\mathrm{Rb} 1 \mathrm{~A}-\mathrm{O} 2^{\mathrm{iv}}$ & $113.71(4)$ & $\mathrm{O} 2^{\mathrm{xv}}-\mathrm{Ga} 1-\mathrm{O} 4^{\mathrm{xvii}}$ & $175.98(6)$ \\
\hline $\mathrm{O} 3-\mathrm{Rb} 1 \mathrm{~A}-\mathrm{O} 2^{\mathrm{iv}}$ & $126.47(4)$ & $\mathrm{O} 2^{\mathrm{ii}}-\mathrm{Ga} 1-\mathrm{O} 4^{\mathrm{xviii}}$ & $88.71(6)$ \\
\hline $\mathrm{Rb} 1 \mathrm{~B}^{\mathrm{i}}-\mathrm{Rb} 1 \mathrm{~A}-\mathrm{O} 2^{\mathrm{ii}}$ & $37.49(3)$ & $\mathrm{O} 2^{\mathrm{xvi}}-\mathrm{Ga} 1-\mathrm{O} 4^{\mathrm{xvii}}$ & $92.25(6)$ \\
\hline $\mathrm{Rb} 1 \mathrm{~B}^{\mathrm{ii}}-\mathrm{Rb} 1 \mathrm{~A}-\mathrm{O} 2^{\mathrm{ii}}$ & $85.56(15)$ & $\mathrm{O} 4^{\mathrm{xvii}}-\mathrm{Ga} 1-\mathrm{O} 4^{\mathrm{xviii}}$ & $87.28(7)$ \\
\hline $\mathrm{O} 3^{\mathrm{iii}}-\mathrm{Rb} 1 \mathrm{~A}-\mathrm{O} 2^{\mathrm{ii}}$ & $64.84(4)$ & $\mathrm{O} 4^{\mathrm{i}}-\mathrm{Ga} 1-\mathrm{O} 4^{\mathrm{xviii}}$ & $87.28(7)$ \\
\hline $\mathrm{O} 3^{\mathrm{iv}}-\mathrm{Rb} 1 \mathrm{~A}-\mathrm{O} 2^{\mathrm{ii}}$ & $115.61(4)$ & $\mathrm{O} 2^{\mathrm{xv}}-\mathrm{Ga} 1-\mathrm{Rb} 2^{\mathrm{xxi}}$ & $124.04(4)$ \\
\hline $\mathrm{O} 3^{\mathrm{ii}}-\mathrm{Rb} 1 \mathrm{~A}-\mathrm{O} 2^{\mathrm{ii}}$ & $48.04(4)$ & $\mathrm{O} 2^{\mathrm{ii}}-\mathrm{Ga} 1-\mathrm{Rb} 2^{\mathrm{xxi}}$ & $124.04(4)$ \\
\hline $\mathrm{O} 3^{\mathrm{i}}-\mathrm{Rb} 1 \mathrm{~A}-\mathrm{O} 2^{\mathrm{ii}}$ & $70.43(4)$ & $\mathrm{O} 2^{\mathrm{xvi}}-\mathrm{Ga} 1-\mathrm{Rb} 2^{\mathrm{xxi}}$ & $124.04(4)$ \\
\hline $\mathrm{O} 3^{\mathrm{v}}-\mathrm{Rb} 1 \mathrm{~A}-\mathrm{O} 2^{\mathrm{ii}}$ & $126.47(4)$ & $\mathrm{O} 4^{\mathrm{xvii}}-\mathrm{Ga} 1-\mathrm{Rb} 2^{\mathrm{xxi}}$ & $52.83(5)$ \\
\hline $\mathrm{O} 3-\mathrm{Rb} 1 \mathrm{~A}-\mathrm{O} 2^{\mathrm{ii}}$ & $113.71(4)$ & $\mathrm{O} 4^{\mathrm{i}}-\mathrm{Ga} 1-\mathrm{Rb} 2^{\mathrm{xxi}}$ & $52.83(5)$ \\
\hline $\mathrm{O} 2^{\mathrm{iv}}-\mathrm{Rb} 1 \mathrm{~A}-\mathrm{O} 2^{\mathrm{ii}}$ & $74.98(5)$ & $\mathrm{O} 4^{\mathrm{xviii}}-\mathrm{Ga} 1-\mathrm{Rb} 2^{\mathrm{xxi}}$ & $52.83(4)$ \\
\hline $\mathrm{Rb} 1 \mathrm{~B}^{\mathrm{i}}-\mathrm{Rb} 1 \mathrm{~A}-\mathrm{O} 2^{\mathrm{v}}$ & $150.57(3)$ & $\mathrm{O} 2^{\mathrm{xv}}-\mathrm{Ga} 1-\mathrm{Rb} 1 \mathrm{~A}^{\mathrm{xvii}}$ & $32.81(4)$ \\
\hline $\mathrm{Rb} 1 \mathrm{~B}^{\mathrm{ii}}-\mathrm{Rb} 1 \mathrm{~A}-\mathrm{O} 2^{\mathrm{v}}$ & $85.56(15)$ & $\mathrm{O} 2^{\mathrm{ii}}-\mathrm{Ga} 1-\mathrm{Rb} 1 \mathrm{~A}^{\mathrm{xvii}}$ & $105.67(4)$ \\
\hline 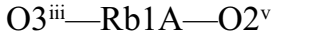 & $113.71(4)$ & $\mathrm{O} 2^{\mathrm{xvi}}-\mathrm{Ga} 1-\mathrm{Rb} 1 \mathrm{~A}^{\mathrm{xvii}}$ & $120.04(5)$ \\
\hline $\mathrm{O}^{\mathrm{iv}}-\mathrm{Rb} 1 \mathrm{~A}-\mathrm{O} 2^{\mathrm{v}}$ & $70.43(4)$ & $\mathrm{O} 4^{\mathrm{xvii}}-\mathrm{Ga} 1-\mathrm{Rb} 1 \mathrm{~A}^{\mathrm{xvii}}$ & $77.51(4)$ \\
\hline 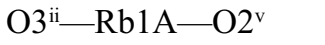 & $126.47(4)$ & $\mathrm{O} 4^{\mathrm{i}}-\mathrm{Ga} 1-\mathrm{Rb} 1 \mathrm{~A}^{\mathrm{xvii}}$ & $59.26(4)$ \\
\hline
\end{tabular}




\begin{tabular}{|c|c|c|c|}
\hline $\mathrm{O} 3^{\mathrm{i}}-\mathrm{Rb} 1 \mathrm{~A}-\mathrm{O} 2^{\mathrm{v}}$ & $115.61(4)$ & $\mathrm{O} 4^{\mathrm{xviii}}-\mathrm{Ga} 1-\mathrm{Rb} 1 \mathrm{~A}^{\mathrm{xvii}}$ & $143.41(5)$ \\
\hline $\mathrm{O} 3^{\mathrm{v}}-\mathrm{Rb} 1 \mathrm{~A}-\mathrm{O} 2^{\mathrm{v}}$ & $48.04(4)$ & $\mathrm{Rb} 2^{\mathrm{xxi}}-\mathrm{Ga} 1-\mathrm{Rb} 1 \mathrm{~A}^{\mathrm{xvii}}$ & $92.384(4)$ \\
\hline $\mathrm{O} 3-\mathrm{Rb} 1 \mathrm{~A}-\mathrm{O} 2^{\mathrm{v}}$ & $64.84(4)$ & $\mathrm{O} 2^{\mathrm{xv}}-\mathrm{Ga} 1-\mathrm{Rb} 1 \mathrm{~A}$ & $120.04(5)$ \\
\hline $\mathrm{O} 2^{\mathrm{iv}}-\mathrm{Rb} 1 \mathrm{~A}-\mathrm{O} 2^{\mathrm{v}}$ & $113.21(2)$ & $\mathrm{O} 22^{\mathrm{ii}}-\mathrm{Ga} 1-\mathrm{Rb} 1 \mathrm{~A}$ & $32.81(4)$ \\
\hline 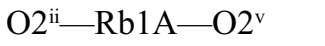 & $171.11(5)$ & $\mathrm{O} 2^{\mathrm{xvi}}-\mathrm{Ga} 1-\mathrm{Rb} 1 \mathrm{~A}$ & $105.67(4)$ \\
\hline $\mathrm{Rb} 1 \mathrm{~B}-\mathrm{Rb} 1 \mathrm{~A}-\mathrm{O} 2$ & $150.57(3)$ & $\mathrm{O} 4^{\mathrm{xvii}}-\mathrm{Ga} 1-\mathrm{Rb} 1 \mathrm{~A}$ & $143.41(5)$ \\
\hline $\mathrm{Rb} 1 \mathrm{~B}^{\mathrm{ii}}-\mathrm{Rb} 1 \mathrm{~A}-\mathrm{O} 2$ & $37.49(14)$ & $\mathrm{O} 4^{\mathrm{i}}-\mathrm{Ga} 1-\mathrm{Rb} 1 \mathrm{~A}$ & $77.51(4)$ \\
\hline 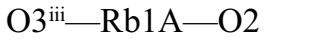 & $115.61(4)$ & 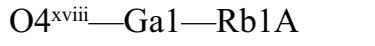 & $59.26(5)$ \\
\hline $\mathrm{O} 3^{\text {iv }}-\mathrm{Rb} 1 \mathrm{~A}-\mathrm{O} 2$ & $126.47(4)$ & $\mathrm{Rb} 2^{\mathrm{xxi}}-\mathrm{Ga} 1-\mathrm{Rb} 1 \mathrm{~A}$ & $92.384(4)$ \\
\hline $\mathrm{O} 3{ }^{\mathrm{ii}}-\mathrm{Rb} 1 \mathrm{~A}-\mathrm{O} 2$ & $70.43(4)$ & $\mathrm{Rb} 1 \mathrm{~A}^{\mathrm{xvii}}-\mathrm{Ga} 1-\mathrm{Rb} 1 \mathrm{~A}$ & $119.828(1)$ \\
\hline $\mathrm{O} 3^{\mathrm{i}}-\mathrm{Rb} 1 \mathrm{~A}-\mathrm{O} 2$ & $113.71(4)$ & $\mathrm{O} 2^{\mathrm{xv}}-\mathrm{Ga} 1-\mathrm{Rb} 1 \mathrm{~A}^{\mathrm{xvi}}$ & $105.67(5)$ \\
\hline $\mathrm{O} 3^{\mathrm{v}}-\mathrm{Rb} 1 \mathrm{~A}-\mathrm{O} 2$ & $64.84(4)$ & $\mathrm{O} 2^{\mathrm{ii}}-\mathrm{Ga} 1-\mathrm{Rb} 1 \mathrm{~A}^{\mathrm{xvi}}$ & $120.04(5)$ \\
\hline $\mathrm{O} 3-\mathrm{Rb} 1 \mathrm{~A}-\mathrm{O} 2$ & $48.04(4)$ & $\mathrm{O} 2^{\mathrm{xvi}}-\mathrm{Ga} 1-\mathrm{Rb} 1 \mathrm{~A}^{\mathrm{xvi}}$ & $32.81(4)$ \\
\hline $\mathrm{O} 2^{\mathrm{iv}}-\mathrm{Rb} 1 \mathrm{~A}-\mathrm{O} 2$ & $171.11(5)$ & $\mathrm{O} 4^{\mathrm{xvii}}-\mathrm{Ga} 1-\mathrm{Rb} 1 \mathrm{~A}^{\mathrm{xvi}}$ & $59.26(4)$ \\
\hline $\mathrm{O} 22^{\mathrm{ii}}-\mathrm{Rb} 1 \mathrm{~A}-\mathrm{O} 2$ & $113.21(2)$ & $\mathrm{O} 44^{\mathrm{i}}-\mathrm{Ga} 1-\mathrm{Rb} \mathrm{A}^{\mathrm{xvi}}$ & $143.41(5)$ \\
\hline $\mathrm{O} 2^{\mathrm{v}}-\mathrm{Rb} 1 \mathrm{~A}-\mathrm{O} 2$ & $58.86(5)$ & $\mathrm{O} 4^{\mathrm{xviii}}-\mathrm{Ga} 1-\mathrm{Rb} \mathrm{A}^{\mathrm{xvi}}$ & $77.51(5)$ \\
\hline $\mathrm{Rb} 1 \mathrm{~B}-\mathrm{Rb} 1 \mathrm{~A}-\mathrm{O} 2^{\mathrm{iii}}$ & $85.56(3)$ & $\mathrm{Rb} 2^{\mathrm{xxi}}-\mathrm{Ga} 1-\mathrm{Rb} 1 \mathrm{~A}^{\mathrm{xvi}}$ & $92.384(4)$ \\
\hline $\mathrm{Rb} 1 \mathrm{~B}^{\mathrm{ii}}-\mathrm{Rb} 1 \mathrm{~A}-\mathrm{O} 2^{\mathrm{iii}}$ & $37.49(14)$ & $\mathrm{Rb} 1 \mathrm{~A}^{\mathrm{xvii}}-\mathrm{Ga} 1-\mathrm{Rb} 1 \mathrm{~A}^{\mathrm{xvi}}$ & $119.828(1)$ \\
\hline $\mathrm{O} 3^{\mathrm{iii}}-\mathrm{Rb} 1 \mathrm{~A}-\mathrm{O} 2^{\mathrm{iii}}$ & $48.04(4)$ & $\mathrm{Rb} 1 \mathrm{~A}-\mathrm{Ga} 1-\mathrm{Rb} 1 \mathrm{~A}^{\mathrm{xvi}}$ & $119.828(1)$ \\
\hline $\mathrm{O} 3^{\mathrm{iv}}-\mathrm{Rb} 1 \mathrm{~A}-\mathrm{O} 2^{\mathrm{iii}}$ & $113.71(4)$ & 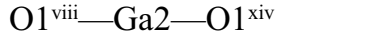 & $93.53(6)$ \\
\hline $\mathrm{O} 3^{\mathrm{ii}}-\mathrm{Rb} 1 \mathrm{~A}-\mathrm{O} 2^{\mathrm{iii}}$ & $64.84(4)$ & 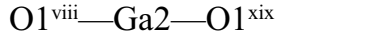 & $93.53(6)$ \\
\hline $\mathrm{O} 3^{\mathrm{i}}-\mathrm{Rb} 1 \mathrm{~A}-\mathrm{O} 2^{\mathrm{iii}}$ & $126.47(4)$ & $\mathrm{O} 1^{\mathrm{xiv}}-\mathrm{Ga} 2-\mathrm{O} 1^{\mathrm{xix}}$ & $93.53(6)$ \\
\hline $\mathrm{O} 3^{\mathrm{v}}-\mathrm{Rb} 1 \mathrm{~A}-\mathrm{O} 2^{\mathrm{iii}}$ & $70.43(4)$ & 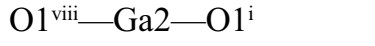 & 180.0 \\
\hline $\mathrm{O} 3-\mathrm{Rb} 1 \mathrm{~A}-\mathrm{O} 2^{\mathrm{iii}}$ & $115.61(4)$ & $\mathrm{O} 1^{\text {xiv }}-\mathrm{Ga} 2-\mathrm{O} 1^{\mathrm{i}}$ & $86.48(6)$ \\
\hline $\mathrm{O} 2^{\mathrm{iv}}-\mathrm{Rb} 1 \mathrm{~A}-\mathrm{O} 2^{\mathrm{iii}}$ & $113.21(2)$ & $\mathrm{O} 1^{\mathrm{xix}}-\mathrm{Ga} 2-\mathrm{O}^{\mathrm{i}}$ & $86.48(6)$ \\
\hline $\mathrm{O} 2^{\mathrm{ii}}-\mathrm{Rb} 1 \mathrm{~A}-\mathrm{O} 2^{\mathrm{iii}}$ & $58.87(5)$ & 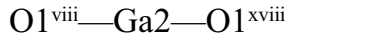 & $86.48(6)$ \\
\hline $\mathrm{O} 2^{\mathrm{v}}-\mathrm{Rb} 1 \mathrm{~A}-\mathrm{O} 2^{\mathrm{iii}}$ & $113.21(2)$ & $\mathrm{O} 1^{\mathrm{xiv}}-\mathrm{Ga} 2-\mathrm{O} 1^{\text {xviii }}$ & 180.0 \\
\hline $\mathrm{O} 2-\mathrm{Rb} 1 \mathrm{~A}-\mathrm{O} 2^{\mathrm{iii}}$ & $74.98(5)$ & $\mathrm{O} 1^{\mathrm{xix}}-\mathrm{Ga} 2-\mathrm{O} 1^{\mathrm{xviii}}$ & $86.48(6)$ \\
\hline $\mathrm{Rb} 1 \mathrm{~B}-\mathrm{Rb} 1 \mathrm{~A}-\mathrm{O} 2^{\mathrm{i}}$ & $85.56(3)$ & $\mathrm{O} 1^{\mathrm{i}}-\mathrm{Ga} 2-\mathrm{O} 1^{\mathrm{xviii}}$ & $93.52(6)$ \\
\hline $\mathrm{Rb} 1 \mathrm{~B}^{\mathrm{ii}}-\mathrm{Rb} 1 \mathrm{~A}-\mathrm{O} 2^{\mathrm{i}}$ & $150.57(14)$ & $\mathrm{O} 1^{\text {viii }-\mathrm{Ga} 2-\mathrm{O} 1^{\mathrm{xvii}}}$ & $86.48(6)$ \\
\hline $\mathrm{O} 3^{\mathrm{iii}}-\mathrm{Rb} 1 \mathrm{~A}-\mathrm{O} 2^{\mathrm{i}}$ & $126.47(4)$ & $\mathrm{O}^{\mathrm{xiv}}-\mathrm{Ga} 2-\mathrm{O} 1^{\mathrm{xvii}}$ & $86.48(6)$ \\
\hline $\mathrm{O} 3^{\mathrm{iv}}-\mathrm{Rb} 1 \mathrm{~A}-\mathrm{O} 2^{\mathrm{i}}$ & $64.84(4)$ & $\mathrm{O}^{\mathrm{xix}}-\mathrm{Ga} 2-\mathrm{O} 1^{\mathrm{xvii}}$ & 180.0 \\
\hline $\mathrm{O} 3^{\mathrm{ii}}-\mathrm{Rb} 1 \mathrm{~A}-\mathrm{O} 2^{\mathrm{i}}$ & $113.71(4)$ & $\mathrm{O} 1^{\mathrm{i}}-\mathrm{Ga} 2-\mathrm{O} 1^{\mathrm{xvii}}$ & $93.52(6)$ \\
\hline $\mathrm{O} 3^{\mathrm{i}}-\mathrm{Rb} 1 \mathrm{~A}-\mathrm{O} 2^{\mathrm{i}}$ & $48.04(4)$ & $\mathrm{O} 1^{\mathrm{xviii}}-\mathrm{Ga} 2-\mathrm{O} 1^{\mathrm{xvii}}$ & $93.52(6)$ \\
\hline $\mathrm{O} 3^{\mathrm{v}}-\mathrm{Rb} 1 \mathrm{~A}-\mathrm{O} 2^{\mathrm{i}}$ & $115.61(4)$ & 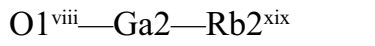 & $63.39(5)$ \\
\hline $\mathrm{O} 3-\mathrm{Rb} 1 \mathrm{~A}-\mathrm{O} 2^{\mathrm{i}}$ & $70.43(4)$ & $\mathrm{O} 1^{\mathrm{xiv}}-\mathrm{Ga} 2-\mathrm{Rb} 2^{\mathrm{xix}}$ & $66.53(5)$ \\
\hline $\mathrm{O} 2^{\mathrm{iv}}-\mathrm{Rb} 1 \mathrm{~A}-\mathrm{O} 2^{\mathrm{i}}$ & $58.87(5)$ & $\mathrm{O} 1^{\mathrm{xix}}-\mathrm{Ga} 2-\mathrm{Rb} 2^{\mathrm{xix}}$ & $146.92(4)$ \\
\hline $\mathrm{O} 2^{\mathrm{ii}}-\mathrm{Rb} 1 \mathrm{~A}-\mathrm{O} 2^{\mathrm{i}}$ & $113.21(2)$ & $\mathrm{O} 1^{\mathrm{i}}-\mathrm{Ga} 2-\mathrm{Rb}^{\mathrm{xix}}$ & $116.61(5)$ \\
\hline $\mathrm{O} 2^{\mathrm{v}}-\mathrm{Rb} 1 \mathrm{~A}-\mathrm{O} 2^{\mathrm{i}}$ & $74.98(5)$ & $\mathrm{O} 1^{\mathrm{xviii}}-\mathrm{Ga} 2-\mathrm{Rb} 2^{\mathrm{xix}}$ & $113.47(5)$ \\
\hline $\mathrm{O} 2-\mathrm{Rb} 1 \mathrm{~A}-\mathrm{O} 2^{\mathrm{i}}$ & $113.21(2)$ & $\mathrm{O} 1^{\mathrm{xvii}}-\mathrm{Ga} 2-\mathrm{Rb} 2^{\mathrm{xix}}$ & $33.08(4)$ \\
\hline $\mathrm{O} 2^{\mathrm{iii}}-\mathrm{Rb} 1 \mathrm{~A}-\mathrm{O} 2^{\mathrm{i}}$ & $171.11(6)$ & $\mathrm{O} 1^{\text {viii }}-\mathrm{Ga} 2-\mathrm{Rb} 2^{\mathrm{xvii}}$ & $116.62(5)$ \\
\hline $\mathrm{Rb} 1 \mathrm{~B}^{\mathrm{i}}-\mathrm{Rb} 1 \mathrm{~A}-\mathrm{As}^{\mathrm{iv}}$ & $60.827(6)$ & $\mathrm{O} 1^{\mathrm{xiv}}-\mathrm{Ga} 2-\mathrm{Rb} 2^{\mathrm{xvii}}$ & $113.48(5)$ \\
\hline $\mathrm{Rb} 1 \mathrm{~B}^{\mathrm{ii}}-\mathrm{Rb} 1 \mathrm{~A}-\mathrm{As}^{\mathrm{iv}}$ & $149.73(2)$ & $\mathrm{O} 1^{\mathrm{xix}}-\mathrm{Ga} 2-\mathrm{Rb} 2^{\mathrm{xvii}}$ & $33.08(4)$ \\
\hline $\mathrm{O} 3^{\mathrm{iii}}-\mathrm{Rb} 1 \mathrm{~A}-\mathrm{As}^{\mathrm{iv}}$ & $73.16(3)$ & $\mathrm{O} 1^{\mathrm{i}}-\mathrm{Ga} 2-\mathrm{Rb} 2^{\mathrm{xvii}}$ & $63.38(5)$ \\
\hline $\mathrm{O}^{3 \mathrm{iv}}-\mathrm{Rb} 1 \mathrm{~A}-\mathrm{As}^{\mathrm{iv}}$ & $24.86(3)$ & $\mathrm{O} 1^{\mathrm{xvii}}-\mathrm{Ga} 2-\mathrm{Rb} 2^{\mathrm{xvii}}$ & $66.52(5)$ \\
\hline $\mathrm{O} 3^{\mathrm{ii}}-\mathrm{Rb} 1 \mathrm{~A}-\mathrm{As}^{\mathrm{iv}}$ & $139.65(3)$ & $\mathrm{O} 1^{\mathrm{xvii}}-\mathrm{Ga} 2-\mathrm{Rb} 2^{\mathrm{xvii}}$ & $146.92(4)$ \\
\hline $\mathrm{O} 3^{\mathrm{i}}-\mathrm{Rb} 1 \mathrm{~A}-\mathrm{As}^{\mathrm{iv}}$ & $79.79(3)$ & $\mathrm{Rb} 2^{\mathrm{xix}}-\mathrm{Ga} 2-\mathrm{Rb} 2^{\mathrm{xvii}}$ & 180.0 \\
\hline $\mathrm{O} 3^{\mathrm{v}}-\mathrm{Rb} 1 \mathrm{~A}-\mathrm{As}^{\mathrm{iv}}$ & $93.74(3)$ & 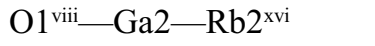 & $113.48(5)$ \\
\hline
\end{tabular}




\begin{tabular}{|c|c|}
\hline $\mathrm{O} 3-\mathrm{Rb} 1 \mathrm{~A}-\mathrm{As}^{\mathrm{iv}}$ & $123.16(3)$ \\
\hline $\mathrm{O} 2^{\mathrm{iv}}-\mathrm{Rb} 1 \mathrm{~A}-\mathrm{As}^{\mathrm{iv}}$ & $24.28(2)$ \\
\hline 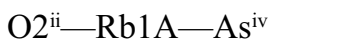 & $97.93(3)$ \\
\hline $\mathrm{O} 2^{\mathrm{v}}-\mathrm{Rb} 1 \mathrm{~A}-\mathrm{As}^{\mathrm{iv}}$ & $89.76(3)$ \\
\hline $\mathrm{O} 2-\mathrm{Rb} 1 \mathrm{~A}-\mathrm{As}^{\mathrm{iv}}$ & $148.57(3)$ \\
\hline $\mathrm{O} 2^{\mathrm{iii}}-\mathrm{Rb} 1 \mathrm{~A}-\mathrm{As}^{\mathrm{iv}}$ & $121.15(3)$ \\
\hline $\mathrm{O} 2^{\mathrm{i}}-\mathrm{Rb} 1 \mathrm{~A}-\mathrm{As}^{\mathrm{iv}}$ & $53.64(3)$ \\
\hline $\mathrm{Rb} 1 \mathrm{~B}^{\mathrm{i}}-\mathrm{Rb} 1 \mathrm{~B}-\mathrm{Rb} 1 \mathrm{~B}^{\mathrm{ii}}$ & $60.00(4)$ \\
\hline $\mathrm{Rb} \mathrm{B}^{\mathrm{i}}-\mathrm{Rb} 1 \mathrm{~B}-\mathrm{O}^{\mathrm{iv}}$ & $94.7(6)$ \\
\hline $\mathrm{Rb} 1 \mathrm{~B}^{\mathrm{ii}-}-\mathrm{Rb} 1 \mathrm{~B}-\mathrm{O}^{\mathrm{iv}}$ & $123.8(6)$ \\
\hline $\mathrm{Rb} 1 \mathrm{~B}^{\mathrm{i}}-\mathrm{Rb} 1 \mathrm{~B}-\mathrm{O} 3$ & $123.8(6)$ \\
\hline $\mathrm{Rb} 1 \mathrm{~B}^{\mathrm{ii}}-\mathrm{Rb} 1 \mathrm{~B}-\mathrm{O} 3$ & $94.7(7)$ \\
\hline $\mathrm{O}{ }^{\mathrm{iv}}-\mathrm{Rb} 1 \mathrm{~B}-\mathrm{O} 3$ & $136.7(14)$ \\
\hline $\mathrm{Rb} 1 \mathrm{~B}^{\mathrm{i}}-\mathrm{Rb} 1 \mathrm{~B}-\mathrm{O} 2^{\mathrm{v}}$ & $160.6(4)$ \\
\hline $\mathrm{Rb} 1 \mathrm{~B}^{\mathrm{ii}}-\mathrm{Rb} 1 \mathrm{~B}-\mathrm{O} 2^{\mathrm{v}}$ & $109.8(6)$ \\
\hline $\mathrm{O}^{3}{ }^{\mathrm{i}}-\mathrm{Rb} 1 \mathrm{~B}-\mathrm{O}_{2}^{\mathrm{v}}$ & $77.3(7)$ \\
\hline $\mathrm{O} 3-\mathrm{Rb} 1 \mathrm{~B}-\mathrm{O} 2^{\mathrm{v}}$ & $71.0(6)$ \\
\hline $\mathrm{Rb} 1 \mathrm{~B}^{\mathrm{i}}-\mathrm{Rb} 1 \mathrm{~B}-\mathrm{O} 2^{\mathrm{i}}$ & $109.8(5)$ \\
\hline $\mathrm{Rb} 1 \mathrm{~B}^{\mathrm{ii}}-\mathrm{Rb} 1 \mathrm{~B}-\mathrm{O} 2^{\mathrm{i}}$ & $160.6(3)$ \\
\hline $\mathrm{O} 3^{\mathrm{i}}-\mathrm{Rb} 1 \mathrm{~B}-\mathrm{O} 2^{\mathrm{i}}$ & $71.0(6)$ \\
\hline $\mathrm{O} 3-\mathrm{Rb} 1 \mathrm{~B}-\mathrm{O} 2^{\mathrm{i}}$ & $77.3(7)$ \\
\hline 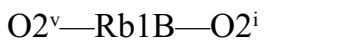 & $84.6(10)$ \\
\hline $\mathrm{Rb} 1 \mathrm{~B}^{\mathrm{i}}-\mathrm{Rb} 1 \mathrm{~B}-\mathrm{O} 3^{\mathrm{i}}$ & $72.1(6)$ \\
\hline $\mathrm{Rb} 1 \mathrm{~B}^{\mathrm{ii}}-\mathrm{Rb} 1 \mathrm{~B}-\mathrm{O}^{\mathrm{i}}$ & $109.8(6)$ \\
\hline $\mathrm{O}^{\mathrm{iv}}-\mathrm{Rb} 1 \mathrm{~B}-\mathrm{O} 3^{\mathrm{i}}$ & $107.2(4)$ \\
\hline $\mathrm{O} 3-\mathrm{Rb} 1 \mathrm{~B}-\mathrm{O}^{\mathrm{i}}$ & $72.0(2)$ \\
\hline $\mathrm{O} 2^{\mathrm{v}}-\mathrm{Rb} 1 \mathrm{~B}-\mathrm{O}^{\mathrm{i}}$ & $127.0(11)$ \\
\hline $\mathrm{O} 2^{\mathrm{i}}-\mathrm{Rb} 1 \mathrm{~B}-\mathrm{O} 3^{\mathrm{i}}$ & $51.0(2)$ \\
\hline $\mathrm{Rb} 1 \mathrm{~B}^{\mathrm{i}}-\mathrm{Rb} 1 \mathrm{~B}-\mathrm{O}^{\mathrm{v}}$ & $109.8(7)$ \\
\hline $\mathrm{Rb}_{1 \mathrm{~B}}{ }^{\mathrm{ii}}-\mathrm{Rb} 1 \mathrm{~B}-\mathrm{O}^{\mathrm{v}}$ & $72.1(7)$ \\
\hline $\mathrm{O}^{3 \mathrm{iv}}-\mathrm{Rb} 1 \mathrm{~B}-\mathrm{O}^{\mathrm{v}}$ & $72.0(2)$ \\
\hline $\mathrm{O} 3-\mathrm{Rb} 1 \mathrm{~B}-\mathrm{O}^{\mathrm{v}}$ & $107.2(4)$ \\
\hline $\mathrm{O} 2^{\mathrm{v}}-\mathrm{Rb} 1 \mathrm{~B}-\mathrm{O}^{\mathrm{v}}$ & $51.0(2)$ \\
\hline $\mathrm{O} 2^{\mathrm{i}}-\mathrm{Rb} 1 \mathrm{~B}-\mathrm{O}^{\mathrm{v}}$ & $127.0(11)$ \\
\hline $\mathrm{O} 3^{\mathrm{i}}-\mathrm{Rb} 1 \mathrm{~B}-\mathrm{O}^{\mathrm{v}}$ & $177.9(14)$ \\
\hline $\mathrm{Rb} 1 \mathrm{~B}^{\mathrm{i}}-\mathrm{Rb} 1 \mathrm{~B}-\mathrm{O} 2^{\mathrm{iv}}$ & $58.5(7)$ \\
\hline 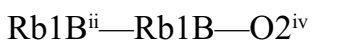 & $116.2(6)$ \\
\hline $\mathrm{O}^{\text {iv }}-\mathrm{Rb} 1 \mathrm{~B}-\mathrm{O} 2^{\mathrm{iv}}$ & $49.25(8)$ \\
\hline $\mathrm{O} 3-\mathrm{Rb} 1 \mathrm{~B}-\mathrm{O} 2^{\mathrm{iv}}$ & $133.4(5)$ \\
\hline $\mathrm{O} 2^{\mathrm{v}}-\mathrm{Rb} 1 \mathrm{~B}-\mathrm{O} 2^{\mathrm{iv}}$ & $122.6(9)$ \\
\hline $\mathrm{O} 2^{\mathrm{i}}-\mathrm{Rb} 1 \mathrm{~B}-\mathrm{O} 2^{\mathrm{iv}}$ & $62.0(3)$ \\
\hline $\mathrm{O} 3^{\mathrm{i}}-\mathrm{Rb} 1 \mathrm{~B}-\mathrm{O} 2^{\mathrm{iv}}$ & $65.28(4)$ \\
\hline $\mathrm{O} 3^{\mathrm{v}}-\mathrm{Rb} 1 \mathrm{~B}-\mathrm{O} 2^{\mathrm{iv}}$ & $114.83(5)$ \\
\hline $\mathrm{Rb} 1 \mathrm{~B}-\mathrm{Rb} 1 \mathrm{~B}-\mathrm{O} 2$ & $116.2(7)$ \\
\hline $\mathrm{Rb} 1 \mathrm{~B}^{\mathrm{ii}}-\mathrm{Rb} 1 \mathrm{~B}-\mathrm{O} 2$ & $58.5(8)$ \\
\hline $\mathrm{O}{ }^{\mathrm{iv}}-\mathrm{Rb} 1 \mathrm{~B}-\mathrm{O} 2$ & $133.4(5)$ \\
\hline $\mathrm{O} 3-\mathrm{Rb} 1 \mathrm{~B}-\mathrm{O} 2$ & $49.25(8)$ \\
\hline $\mathrm{O} 2 \mathrm{v}-\mathrm{Rb} 1 \mathrm{~B}-\mathrm{O} 2$ & $62.0(3)$ \\
\hline
\end{tabular}

\begin{tabular}{|c|c|}
\hline $\mathrm{O}^{\mathrm{xiv}}-\mathrm{Ga} 2-\mathrm{Rb} 2^{\mathrm{xvi}}$ & $33.08(4)$ \\
\hline $\mathrm{O} 1^{\mathrm{xix}}-\mathrm{Ga} 2-\mathrm{Rb} 2^{\mathrm{xvi}}$ & $116.62(6)$ \\
\hline 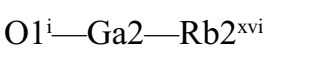 & $66.52(5)$ \\
\hline $\mathrm{O} 1^{\mathrm{xviii}}-\mathrm{Ga} 2-\mathrm{Rb} 2^{\mathrm{xvi}}$ & $146.92(4)$ \\
\hline $\mathrm{O} 1^{\mathrm{xvi}}-\mathrm{Ga} 2-\mathrm{Rb}^{2 \mathrm{xvi}}$ & $63.38(6)$ \\
\hline $\mathrm{Rb} 2^{\mathrm{xix}}-\mathrm{Ga} 2-\mathrm{Rb} 2^{\mathrm{xvi}}$ & 60.0 \\
\hline $\mathrm{Rb} 2^{\mathrm{xvi}}-\mathrm{Ga} 2-\mathrm{Rb} 2^{\mathrm{xvi}}$ & 120.0 \\
\hline $\mathrm{O} 1^{\mathrm{viii}}-\mathrm{Ga} 2-\mathrm{Rb} 2$ & $33.08(5)$ \\
\hline $\mathrm{O} 1^{\mathrm{xi}}-\mathrm{Ga} 2-\mathrm{Rb} 2$ & $116.62(5)$ \\
\hline $\mathrm{O} 1{ }^{\mathrm{xix}}-\mathrm{Ga} 2-\mathrm{Rb} 2$ & $113.48(5)$ \\
\hline $\mathrm{O} 1 \mathrm{i}-\mathrm{Ga} 2-\mathrm{Rb} 2$ & $146.92(5)$ \\
\hline $\mathrm{O} 1^{\mathrm{x} v i i}-\mathrm{Ga} 2-\mathrm{Rb} 2$ & $63.38(5)$ \\
\hline 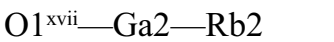 & $66.52(5)$ \\
\hline $\mathrm{Rb} 2^{\mathrm{xix}}-\mathrm{Ga} 2-\mathrm{Rb} 2$ & 60.0 \\
\hline $\mathrm{Rb} 2^{\mathrm{xvii}}-\mathrm{Ga} 2-\mathrm{Rb} 2$ & 120.0 \\
\hline $\mathrm{Rb} 2^{\mathrm{xvi}}-\mathrm{Ga} 2-\mathrm{Rb} 2$ & 120.0 \\
\hline $\mathrm{O} 1^{\text {viii }}-\mathrm{Ga} 2-\mathrm{Rb} 2^{\mathrm{xxii}}$ & $66.52(5)$ \\
\hline $\mathrm{O} 1^{\mathrm{xiv}}-\mathrm{Ga} 2-\mathrm{Rb} 2^{\mathrm{xxii}}$ & $146.92(4)$ \\
\hline $\mathrm{O} 1^{\mathrm{xix}}-\mathrm{Ga} 2-\mathrm{Rb} 2^{\mathrm{xxii}}$ & $63.38(6)$ \\
\hline 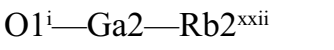 & $113.47(5)$ \\
\hline $\mathrm{O}^{1}{ }^{\mathrm{xiii}}-\mathrm{Ga} 2-\mathrm{Rb}^{\mathrm{xxii}}$ & $33.08(4)$ \\
\hline $\mathrm{O} 1^{\mathrm{xvii}}-\mathrm{Ga} 2-\mathrm{Rb} 2^{\mathrm{xxii}}$ & $116.62(6)$ \\
\hline $\mathrm{Rb} 2^{\mathrm{xix}}-\mathrm{Ga} 2-\mathrm{Rb} 2^{\mathrm{xxii}}$ & 120.0 \\
\hline $\mathrm{Rb} 2^{\mathrm{xvii}}-\mathrm{Ga} 2-\mathrm{Rb} 2^{\mathrm{xxii}}$ & 60.0 \\
\hline $\mathrm{Rb} 2^{\mathrm{xvi}}-\mathrm{Ga} 2-\mathrm{Rb}^{\mathrm{xxii}}$ & 180.0 \\
\hline $\mathrm{Rb} 2-\mathrm{Ga} 2-\mathrm{Rb} 2^{\mathrm{xxii}}$ & 60.0 \\
\hline $\mathrm{O} 1^{\mathrm{viii}}-\mathrm{Ga} 2-\mathrm{Rb} 2^{\mathrm{xxiii}}$ & $146.92(5)$ \\
\hline $\mathrm{O} 1^{\mathrm{xiv}}-\mathrm{Ga} 2-\mathrm{Rb} 2^{\mathrm{xxiii}}$ & $63.38(5)$ \\
\hline $\mathrm{O} 1^{\mathrm{xix}}-\mathrm{Ga} 2-\mathrm{Rb} 2^{\mathrm{xxiii}}$ & $66.52(5)$ \\
\hline $\mathrm{O} 11^{\mathrm{i}}-\mathrm{Ga} 2-\mathrm{Rb} 2^{\mathrm{xxiii}}$ & $33.08(5)$ \\
\hline $\mathrm{O} 1^{\mathrm{xviii}}-\mathrm{Ga} 2-\mathrm{Rb} 2^{\mathrm{xxiii}}$ & $116.61(5)$ \\
\hline $\mathrm{O} 1^{\mathrm{xvii}}-\mathrm{Ga} 2-\mathrm{Rb} 2^{\mathrm{xxiii}}$ & $113.47(5)$ \\
\hline $\mathrm{Rb} 2^{\mathrm{xix}}-\mathrm{Ga} 2-\mathrm{Rb} 2^{\mathrm{xxiii}}$ & 120.0 \\
\hline $\mathrm{Rb} 2^{\mathrm{xvii}}-\mathrm{Ga} 2-\mathrm{Rb} 2^{\mathrm{xxiii}}$ & 60.0 \\
\hline $\mathrm{Rb} 2^{\mathrm{xvi}}-\mathrm{Ga} 2-\mathrm{Rb} 2^{\mathrm{xxiii}}$ & 60.0 \\
\hline $\mathrm{Rb} 2-\mathrm{Ga} 2-\mathrm{Rb} 2^{\mathrm{xxiii}}$ & 180.0 \\
\hline $\mathrm{Rb} 2^{\mathrm{xxii}}-\mathrm{Ga} 2-\mathrm{Rb} 2^{\mathrm{xxiii}}$ & $119.997(1)$ \\
\hline $\mathrm{O} 1^{\mathrm{xx}}-\mathrm{As}-\mathrm{O} 2$ & $119.20(8)$ \\
\hline $\mathrm{O} 1^{\mathrm{xx}}-\mathrm{As}-\mathrm{O} 4^{\mathrm{iv}}$ & $105.45(8)$ \\
\hline $\mathrm{O} 2-\mathrm{As}-\mathrm{O} 4^{\mathrm{iv}}$ & $114.88(7)$ \\
\hline $\mathrm{O} 1^{\mathrm{xx}}-\mathrm{As}-\mathrm{O} 3$ & $107.00(9)$ \\
\hline $\mathrm{O} 2-\mathrm{As}-\mathrm{O} 3$ & $103.30(8)$ \\
\hline $\mathrm{O} 4^{\mathrm{iv}}-\mathrm{As}-\mathrm{O} 3$ & $106.04(8)$ \\
\hline $\mathrm{O} 1^{\mathrm{xx}}-\mathrm{As}-\mathrm{Rb} 2^{\mathrm{xii}}$ & $64.25(6)$ \\
\hline $\mathrm{O} 2-\mathrm{As}-\mathrm{Rb} 2^{\mathrm{xii}}$ & $172.80(5)$ \\
\hline $\mathrm{O} 4^{\mathrm{iv}}-\mathrm{As}-\mathrm{Rb} 2^{\mathrm{xii}}$ & $68.49(5)$ \\
\hline $\mathrm{O} 3-\mathrm{As}-\mathrm{Rb} 2^{\mathrm{xii}}$ & $69.51(6)$ \\
\hline $\mathrm{O} 1^{\mathrm{xx}}-\mathrm{As}-\mathrm{Rb} 1 \mathrm{~B}^{\mathrm{ii}}$ & $142.0(2)$ \\
\hline
\end{tabular}




\begin{tabular}{|c|c|}
\hline $\mathrm{O} 2^{\mathrm{i}}-\mathrm{Rb} 1 \mathrm{~B}-\mathrm{O} 2$ & $122.6(9)$ \\
\hline 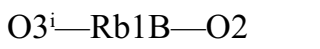 & $114.83(5)$ \\
\hline $\mathrm{O}^{\mathrm{v}}-\mathrm{Rb} 1 \mathrm{~B}-\mathrm{O} 2$ & $65.28(4)$ \\
\hline $\mathrm{O} 2^{\mathrm{iv}}-\mathrm{Rb} 1 \mathrm{~B}-\mathrm{O} 2$ & $174.7(13)$ \\
\hline $\mathrm{Rb} 1 \mathrm{~B}^{\mathrm{i}}-\mathrm{Rb} 1 \mathrm{~B}-\mathrm{O} 3^{\mathrm{iii}}$ & $46.2(3)$ \\
\hline $\mathrm{Rb} 1 \mathrm{~B}^{\mathrm{ii}}-\mathrm{Rb} 1 \mathrm{~B}-\mathrm{O} 3^{\mathrm{iii}}$ & $58.9(3)$ \\
\hline $\mathrm{O}^{\mathrm{iv}}-\mathrm{Rb} 1 \mathrm{~B}-\mathrm{O} 3^{\mathrm{iii}}$ & $67.6(2)$ \\
\hline $\mathrm{O} 3-\mathrm{Rb} 1 \mathrm{~B}-\mathrm{O} 3^{\mathrm{iii}}$ & $153.6(10)$ \\
\hline $\mathrm{O} 2^{\mathrm{v}}-\mathrm{Rb} 1 \mathrm{~B}-\mathrm{O} 3^{\mathrm{iii}}$ & $114.75(4)$ \\
\hline $\mathrm{O} 2^{\mathrm{i}}-\mathrm{Rb} 1 \mathrm{~B}-\mathrm{O} 3^{\mathrm{iii}}$ & $127.90(5)$ \\
\hline 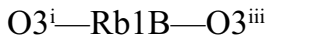 & $115.4(7)$ \\
\hline $\mathrm{O}^{\mathrm{v}}-\mathrm{Rb} 1 \mathrm{~B}-\mathrm{O} 3^{\mathrm{iii}}$ & $66.2(3)$ \\
\hline $\mathrm{O} 2^{\mathrm{iv}}-\mathrm{Rb} 1 \mathrm{~B}-\mathrm{O} 3^{\mathrm{iii}}$ & $67.3(3)$ \\
\hline $\mathrm{O} 2-\mathrm{Rb} 1 \mathrm{~B}-\mathrm{O} 3^{\mathrm{iii}}$ & $108.7(7)$ \\
\hline $\mathrm{Rb} 1 \mathrm{~B}^{\mathrm{i}}-\mathrm{Rb} 1 \mathrm{~B}-\mathrm{O}^{\mathrm{ii}}$ & $58.9(4)$ \\
\hline $\mathrm{Rb} 1 \mathrm{~B}^{\mathrm{ii}}-\mathrm{Rb} 1 \mathrm{~B}-\mathrm{O} 3^{\mathrm{ii}}$ & $46.2(3)$ \\
\hline $\mathrm{O}^{\mathrm{iv}}-\mathrm{Rb} 1 \mathrm{~B}-\mathrm{O} 3^{\mathrm{ii}}$ & $153.6(10)$ \\
\hline $\mathrm{O} 3-\mathrm{Rb} 1 \mathrm{~B}-\mathrm{O} 3^{3 i}$ & $67.6(2)$ \\
\hline $\mathrm{O} 2^{\mathrm{v}}-\mathrm{Rb} 1 \mathrm{~B}-\mathrm{O} 3^{\mathrm{ii}}$ & $127.90(5)$ \\
\hline $\mathrm{O} 2^{\mathrm{i}}-\mathrm{Rb} 1 \mathrm{~B}-\mathrm{O} 3^{\mathrm{ii}}$ & $114.75(4)$ \\
\hline $\mathrm{O} 3^{\mathrm{i}}-\mathrm{Rb} 1 \mathrm{~B}-\mathrm{O} 3^{\mathrm{ii}}$ & $66.2(3)$ \\
\hline $\mathrm{O}^{2}-\mathrm{Rb} 1 \mathrm{~B}-\mathrm{O}^{\mathrm{ii}}$ & $115.4(7)$ \\
\hline $\mathrm{O} 2^{\mathrm{iv}}-\mathrm{Rb} 1 \mathrm{~B}-\mathrm{O} 3^{\mathrm{ii}}$ & $108.7(7)$ \\
\hline $\mathrm{O} 2-\mathrm{Rb} 1 \mathrm{~B}-\mathrm{O} 3^{3 i}$ & $67.3(3)$ \\
\hline 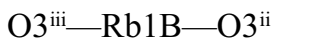 & $91.5(9)$ \\
\hline $\mathrm{O} 3-\mathrm{Rb} 2-\mathrm{O}^{\mathrm{i}}$ & $76.48(6)$ \\
\hline $\mathrm{O} 3-\mathrm{Rb} 2-\mathrm{O}^{\mathrm{ii}}$ & $76.48(6)$ \\
\hline $\mathrm{O} 3^{\mathrm{i}}-\mathrm{Rb} 2-\mathrm{O} 3^{\mathrm{ii}}$ & $76.48(6)$ \\
\hline $\mathrm{O} 3-\mathrm{Rb} 2-\mathrm{O} 1^{\mathrm{vi}}$ & $91.00(4)$ \\
\hline $\mathrm{O}^{\mathrm{i}}-\mathrm{Rb} 2-\mathrm{O}^{\mathrm{vi}}$ & $76.80(4)$ \\
\hline $\mathrm{O}^{3 i}-\mathrm{Rb} 2-\mathrm{O}^{\mathrm{ii}}$ & $152.49(5)$ \\
\hline $\mathrm{O} 3-\mathrm{Rb} 2-\mathrm{O} 1^{\mathrm{vii}}$ & $76.80(5)$ \\
\hline 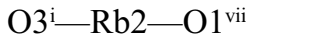 & $152.49(5)$ \\
\hline $\mathrm{O}^{3}{ }^{\mathrm{ii}}-\mathrm{Rb} 2-\mathrm{O} 1^{\mathrm{vii}}$ & $91.00(4)$ \\
\hline 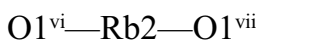 & $110.14(3)$ \\
\hline $\mathrm{O} 3-\mathrm{Rb} 2-\mathrm{O}^{\mathrm{vii}}$ & $152.49(5)$ \\
\hline 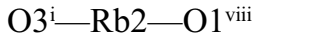 & $91.00(5)$ \\
\hline 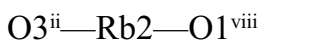 & $76.80(5)$ \\
\hline 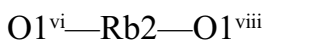 & $110.14(3)$ \\
\hline 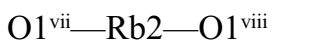 & $110.13(2)$ \\
\hline $\mathrm{O} 3-\mathrm{Rb} 2-\mathrm{O} 4^{\mathrm{ix}}$ & $126.84(4)$ \\
\hline 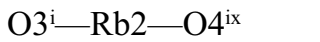 & $111.15(5)$ \\
\hline 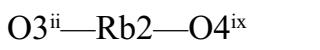 & $156.15(4)$ \\
\hline 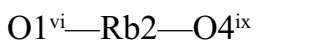 & $45.47(4)$ \\
\hline $\mathrm{O} 1^{\mathrm{vii}-\mathrm{Rb} 2-\mathrm{O} 4^{\mathrm{ix}}}$ & $90.21(4)$ \\
\hline $\mathrm{O} 1^{\mathrm{viii}-\mathrm{Rb} 2-\mathrm{O} 4^{\mathrm{ix}}}$ & $80.43(4)$ \\
\hline $\mathrm{O} 3-\mathrm{Rb} 2-\mathrm{O} 4^{\mathrm{x}}$ & $111.15(5)$ \\
\hline 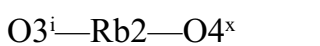 & 156.15 \\
\hline
\end{tabular}

\begin{tabular}{|c|c|}
\hline $\mathrm{O} 2-\mathrm{As}-\mathrm{Rb} 1 \mathrm{~B}^{\mathrm{ii}}$ & $50.6(6)$ \\
\hline $\mathrm{O} 4^{\mathrm{iv}}-\mathrm{As}-\mathrm{Rb} 1 \mathrm{~B}^{\mathrm{ii}}$ & $111.5(3)$ \\
\hline $\mathrm{O} 3-\mathrm{As}-\mathrm{Rb} 1 \mathrm{~B}^{\mathrm{ii}}$ & $54.9(5)$ \\
\hline $\mathrm{Rb} 2^{\mathrm{xii}}-\mathrm{As}-\mathrm{Rb} 1 \mathrm{~B}^{\mathrm{ii}}$ & $122.5(5)$ \\
\hline $\mathrm{O}^{\mathrm{xx}}-\mathrm{As}-\mathrm{Rb} 1 \mathrm{~B}$ & $146.8(4)$ \\
\hline $\mathrm{O} 2-\mathrm{As}-\mathrm{Rb} 1 \mathrm{~B}$ & $60.0(4)$ \\
\hline $\mathrm{O} 4^{\mathrm{iv}}-\mathrm{As}-\mathrm{Rb} 1 \mathrm{~B}$ & $103.6(5)$ \\
\hline $\mathrm{O} 3-\mathrm{As}-\mathrm{Rb} 1 \mathrm{~B}$ & $48.68(19)$ \\
\hline $\mathrm{Rb} 2^{\mathrm{xii}}-\mathrm{As}-\mathrm{Rb} 1 \mathrm{~B}$ & $113.4(4)$ \\
\hline $\mathrm{Rb} 1 \mathrm{~B}^{\mathrm{ii}}-\mathrm{As}-\mathrm{Rb} 1 \mathrm{~B}$ & $10.8(11)$ \\
\hline $\mathrm{O} 1^{\mathrm{xx}}-\mathrm{As}-\mathrm{Rb} 1 \mathrm{~A}$ & $143.22(6)$ \\
\hline $\mathrm{O} 2-\mathrm{As}-\mathrm{Rb} 1 \mathrm{~A}$ & $55.94(5)$ \\
\hline $\mathrm{O} 4^{\mathrm{iv}}-\mathrm{As}-\mathrm{Rb} 1 \mathrm{~A}$ & $108.77(5)$ \\
\hline $\mathrm{O} 3-\mathrm{As}-\mathrm{Rb} 1 \mathrm{~A}$ & $50.50(6)$ \\
\hline $\mathrm{Rb} 2^{\mathrm{xii}}-\mathrm{As}-\mathrm{Rb} 1 \mathrm{~A}$ & $117.262(8)$ \\
\hline $\mathrm{Rb} 1 \mathrm{~B}^{\mathrm{ii}}-\mathrm{As}-\mathrm{Rb} 1 \mathrm{~A}$ & $5.5(5)$ \\
\hline $\mathrm{Rb} 1 \mathrm{~B}-\mathrm{As}-\mathrm{Rb} 1 \mathrm{~A}$ & $5.7(6)$ \\
\hline $\mathrm{O} 1^{\mathrm{xx}}-\mathrm{As}-\mathrm{Rb} 2$ & $81.53(7)$ \\
\hline $\mathrm{O} 2-\mathrm{As}-\mathrm{Rb} 2$ & $99.68(5)$ \\
\hline $\mathrm{O} 4^{\mathrm{iv}}-\mathrm{As}-\mathrm{Rb} 2$ & $133.31(5)$ \\
\hline $\mathrm{O} 3-\mathrm{As}-\mathrm{Rb} 2$ & $32.31(6)$ \\
\hline $\mathrm{Rb}^{2 \mathrm{xi}}-\mathrm{As}-\mathrm{Rb} 2$ & $74.193(8)$ \\
\hline $\mathrm{Rb} 1 \mathrm{~B}^{\mathrm{ii}}-\mathrm{As}-\mathrm{Rb} 2$ & $67.2(2)$ \\
\hline $\mathrm{Rb} 1 \mathrm{~B}-\mathrm{As}-\mathrm{Rb} 2$ & $66.79(16)$ \\
\hline $\mathrm{Rb} 1 \mathrm{~A}-\mathrm{As}-\mathrm{Rb} 2$ & $65.327(14)$ \\
\hline $\mathrm{O} 1^{\mathrm{xx}}-\mathrm{As}-\mathrm{Rb} 1^{\mathrm{xxiv}}$ & $87.84(7)$ \\
\hline $\mathrm{O} 2-\mathrm{As}-\mathrm{Rb} 1 \mathrm{~A}^{\mathrm{xxiv}}$ & $94.28(5)$ \\
\hline $\mathrm{O} 4^{\mathrm{iv}}-\mathrm{As}-\mathrm{Rb} 1 \mathrm{~A}^{\mathrm{xxiv}}$ & $40.78(5)$ \\
\hline $\mathrm{O} 3-\mathrm{As}-\mathrm{Rb} 1 \mathrm{~A}^{\mathrm{xxiv}}$ & $146.81(6)$ \\
\hline $\mathrm{Rb} 2^{\mathrm{xii}}-\mathrm{As}-\mathrm{Rb} 1 \mathrm{~A}^{\mathrm{xxiv}}$ & $92.146(8)$ \\
\hline $\mathrm{Rb} 1 \mathrm{~B}^{\mathrm{ii}}-\mathrm{As}-\mathrm{Rb} 1 \mathrm{~A}^{\mathrm{xxiv}}$ & $126.24(14)$ \\
\hline $\mathrm{Rb} 1 \mathrm{~B}-\mathrm{As}-\mathrm{Rb} 1 \mathrm{~A}^{\mathrm{xxiv}}$ & $125.1(3)$ \\
\hline $\mathrm{Rb} 1 \mathrm{~A}-\mathrm{As}-\mathrm{Rb} 1 \mathrm{~A}^{\mathrm{xxiv}}$ & $127.415(12)$ \\
\hline $\mathrm{Rb} 2-\mathrm{As}-\mathrm{Rb} 1 \mathrm{~A}^{\mathrm{xxiv}}$ & $165.357(7)$ \\
\hline $\mathrm{O} 1^{\mathrm{xx}}-\mathrm{As}-\mathrm{Rb} 2^{\mathrm{xxiv}}$ & $42.87(6)$ \\
\hline $\mathrm{O} 2-\mathrm{As}-\mathrm{Rb} 2^{\mathrm{xxiv}}$ & $127.54(5)$ \\
\hline $\mathrm{O} 4^{\mathrm{iv}}-\mathrm{As}-\mathrm{Rb} 2^{\mathrm{xxiv}}$ & $64.16(5)$ \\
\hline $\mathrm{O} 3-\mathrm{As}-\mathrm{Rb} 2^{\mathrm{xxiv}}$ & $128.20(6)$ \\
\hline $\mathrm{Rb} 2^{\mathrm{xii}}-\mathrm{As}-\mathrm{Rb} 2^{\mathrm{xxiv}}$ & $59.507(5)$ \\
\hline $\mathrm{Rb} 1 \mathrm{~B}^{\mathrm{ii}}-\mathrm{As}-\mathrm{Rb} 2^{\mathrm{xxiv}}$ & $174.80(5)$ \\
\hline $\mathrm{Rb} 1 \mathrm{~B}-\mathrm{As}-\mathrm{Rb} 2^{\mathrm{xxiv}}$ & $167.1(6)$ \\
\hline $\mathrm{Rb} 1 \mathrm{~A}-\mathrm{As}-\mathrm{Rb} 2^{\mathrm{xxiv}}$ & $172.736(5)$ \\
\hline $\mathrm{Rb} 2-\mathrm{As}-\mathrm{Rb} 2^{\mathrm{xxiv}}$ & $117.769(15)$ \\
\hline $\mathrm{Rb} 1 \mathrm{~A}^{\mathrm{xxi}}-\mathrm{As}-\mathrm{Rb} 2^{\mathrm{xxiv}}$ & $48.647(11)$ \\
\hline $\mathrm{As}{ }^{\mathrm{xxv}}-\mathrm{O} 1-\mathrm{Ga}^{\mathrm{xxvi}}$ & $137.45(10)$ \\
\hline $\mathrm{As}^{\mathrm{xxv}}-\mathrm{O} 1-\mathrm{Rb} 2^{\mathrm{vi}}$ & $89.47(6)$ \\
\hline $\mathrm{Ga} 2^{\mathrm{xxvi}}-\mathrm{O} 1-\mathrm{Rb} 2^{\mathrm{vi}}$ & $128.38(6)$ \\
\hline $\mathrm{As}^{\mathrm{xxv}}-\mathrm{O} 1-\mathrm{Rb}^{\mathrm{xxv}}$ & $76.24(6)$ \\
\hline
\end{tabular}




\begin{tabular}{|c|c|}
\hline $\mathrm{O} 3^{\mathrm{ii}}-\mathrm{Rb} 2-\mathrm{O}^{\mathrm{x}}$ & $126.84(4)$ \\
\hline 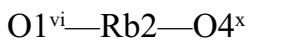 & $80.43(4)$ \\
\hline $\mathrm{O} 1^{\mathrm{vii}-\mathrm{Rb} 2-\mathrm{O} 4^{\mathrm{x}}}$ & $45.46(4)$ \\
\hline $\mathrm{O} 1^{\mathrm{vii}-\mathrm{Rb} 2-\mathrm{O} 4^{\mathrm{x}}}$ & $90.21(4)$ \\
\hline $\mathrm{O} 4^{\mathrm{ix}}-\mathrm{Rb} 2-\mathrm{O} 4^{\mathrm{x}}$ & $45.74(4)$ \\
\hline $\mathrm{O} 3-\mathrm{Rb} 2-\mathrm{O} 4^{\mathrm{xi}}$ & $156.15(5)$ \\
\hline $\mathrm{O} 3^{\mathrm{i}}-\mathrm{Rb} 2-\mathrm{O}^{\mathrm{xi}}$ & $126.84(5)$ \\
\hline $\mathrm{O} 3^{\mathrm{ii}}-\mathrm{Rb} 2-\mathrm{O}^{\mathrm{xi}}$ & $111.15(5)$ \\
\hline $\mathrm{O} 1^{\mathrm{vi}}-\mathrm{Rb} 2-\mathrm{O} 4^{\mathrm{xi}}$ & $90.21(4)$ \\
\hline 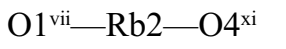 & $80.43(4)$ \\
\hline 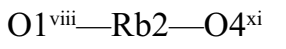 & $45.46(4)$ \\
\hline $\mathrm{O} 4^{\mathrm{ix}}-\mathrm{Rb} 2-\mathrm{O} 4^{\mathrm{xi}}$ & $45.74(4)$ \\
\hline $\mathrm{O} 4^{\mathrm{x}}-\mathrm{Rb} 2-\mathrm{O} 4^{\mathrm{xi}}$ & $45.74(4)$ \\
\hline $\mathrm{O} 3-\mathrm{Rb} 2-\mathrm{O} 3^{\mathrm{xii}}$ & $83.50(5)$ \\
\hline 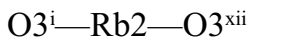 & $119.25(6)$ \\
\hline $\mathrm{O} 3^{\mathrm{ii}}-\mathrm{Rb} 2-\mathrm{O}^{\mathrm{xii}}$ & $150.88(6)$ \\
\hline 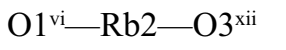 & $46.57(4)$ \\
\hline 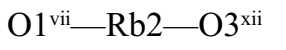 & $63.66(4)$ \\
\hline 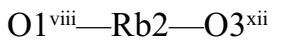 & $123.76(4)$ \\
\hline $\mathrm{O} 4^{\mathrm{ix}}-\mathrm{Rb} 2-\mathrm{O} 3^{\mathrm{xii}}$ & $45.78(4)$ \\
\hline $\mathrm{O} 4^{\mathrm{x}}-\mathrm{Rb} 2-\mathrm{O} 3^{\mathrm{xii}}$ & $43.39(4)$ \\
\hline $\mathrm{O} 4^{\mathrm{xi}}-\mathrm{Rb} 2-\mathrm{O} 3^{\mathrm{xii}}$ & $80.11(4)$ \\
\hline $\mathrm{O} 3-\mathrm{Rb} 2-\mathrm{O} 3^{\mathrm{xiii}}$ & $150.89(6)$ \\
\hline $\mathrm{O} 3^{\mathrm{i}}-\mathrm{Rb} 2-\mathrm{O}^{\mathrm{xiii}}$ & $83.50(5)$ \\
\hline $\mathrm{O} 3^{\mathrm{ii}}-\mathrm{Rb} 2-\mathrm{O} 3^{\mathrm{xiii}}$ & $119.25(6)$ \\
\hline 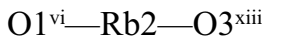 & $63.66(4)$ \\
\hline $\mathrm{O} 1^{\mathrm{vii}}-\mathrm{Rb} 2-\mathrm{O} 3^{\mathrm{xii}}$ & $123.75(4)$ \\
\hline $\mathrm{O} 1^{\text {viii }}-\mathrm{Rb} 2-\mathrm{O} 3^{\text {xiii }}$ & $46.57(4)$ \\
\hline $\mathrm{O} 4^{\mathrm{ix}}-\mathrm{Rb} 2-\mathrm{O} 3^{\mathrm{xiii}}$ & $43.39(4)$ \\
\hline $\mathrm{O} 4^{\mathrm{x}}-\mathrm{Rb} 2-\mathrm{O} 3^{\mathrm{xiii}}$ & $80.11(4)$ \\
\hline $\mathrm{O} 4^{\mathrm{xi}}-\mathrm{Rb} 2-\mathrm{O} 3^{\mathrm{xiii}}$ & $45.78(4)$ \\
\hline $\mathrm{O} 3^{\mathrm{xii}}-\mathrm{Rb} 2-\mathrm{O} 3^{\mathrm{xii}}$ & $88.19(4)$ \\
\hline $\mathrm{O} 3-\mathrm{Rb} 2-\mathrm{O}^{\mathrm{xiv}}$ & $119.25(6)$ \\
\hline 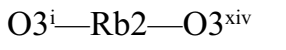 & $150.88(6)$ \\
\hline $\mathrm{O} 3^{\mathrm{ii}}-\mathrm{Rb} 2-\mathrm{O} 3^{\mathrm{xiv}}$ & $83.50(5)$ \\
\hline 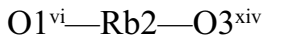 & $123.76(4)$ \\
\hline $\mathrm{O} 1^{\mathrm{vii}}-\mathrm{Rb} 2-\mathrm{O} 3^{\mathrm{xiv}}$ & $46.57(4)$ \\
\hline $\mathrm{O} 1^{\mathrm{vii}-\mathrm{Rb} 2-\mathrm{O} 3^{\mathrm{xiv}}}$ & $63.66(4)$ \\
\hline $\mathrm{O} 4^{\mathrm{ix}}-\mathrm{Rb} 2-\mathrm{O} 3^{\mathrm{xiv}}$ & $80.11(4)$ \\
\hline $\mathrm{O} 4^{\mathrm{x}}-\mathrm{Rb} 2-\mathrm{O} 3^{\mathrm{xiv}}$ & $45.78(4)$ \\
\hline $\mathrm{O} 4^{\mathrm{xi}}-\mathrm{Rb} 2-\mathrm{O}^{\mathrm{xiv}}$ & $43.38(4)$ \\
\hline $\mathrm{O} 3^{\mathrm{xii}}-\mathrm{Rb} 2-\mathrm{O} 3^{\mathrm{xiv}}$ & $88.19(4)$ \\
\hline $\mathrm{O} 3^{\mathrm{xiii}}-\mathrm{Rb} 2-\mathrm{O}^{\mathrm{xiv}}$ & $88.19(4)$ \\
\hline $\mathrm{O} 3-\mathrm{Rb} 2-\mathrm{As}^{\mathrm{xiv}}$ & $102.80(4)$ \\
\hline $\mathrm{O} 3^{\mathrm{i}}-\mathrm{Rb} 2-\mathrm{As}^{\mathrm{xiv}}$ & $177.27(4)$ \\
\hline $\mathrm{O} 3^{\mathrm{ii}-\mathrm{Rb} 2-\mathrm{As}^{\mathrm{xiv}}}$ & $100.79(4)$ \\
\hline $\mathrm{O} 1^{\mathrm{vi}}-\mathrm{Rb} 2-\mathrm{As}^{\mathrm{xiv}}$ & $105.88(3)$ \\
\hline $\mathrm{O} 1^{\mathrm{vii}-\mathrm{Rb} 2-\mathrm{As}^{\mathrm{xiv}}}$ & $26.29(3)$ \\
\hline
\end{tabular}

\begin{tabular}{|c|c|}
\hline $\mathrm{Ga} 2^{\mathrm{xxv}}-\mathrm{O} 1-\mathrm{Rb} 2^{\mathrm{xxv}}$ & $92.73(6)$ \\
\hline $\mathrm{Rb} 2^{\mathrm{vi}}-\mathrm{O} 1-\mathrm{Rb} 2^{\mathrm{xxv}}$ & $76.74(3)$ \\
\hline $\mathrm{As}^{\mathrm{xxv}}-\mathrm{O} 1-\mathrm{Rb}^{\mathrm{xxvi}}$ & $122.41(7)$ \\
\hline $\mathrm{Ga} 2^{\mathrm{xxvi}}-\mathrm{O} 1-\mathrm{Rb} 2^{\mathrm{xxvi}}$ & $89.56(6)$ \\
\hline $\mathrm{Rb}^{\mathrm{vi}}-\mathrm{O} 1-\mathrm{Rb} 2^{\mathrm{xxvi}}$ & $75.20(3)$ \\
\hline $\mathrm{Rb} 2^{\mathrm{xx}}-\mathrm{O} 1-\mathrm{Rb} 2^{\mathrm{xxvi}}$ & $145.76(4)$ \\
\hline As- $\mathrm{O} 2-\mathrm{Ga}^{\mathrm{xxiv}}$ & $121.66(8)$ \\
\hline As $-\mathrm{O} 2-\mathrm{Rb} 1 \mathrm{~B}^{\mathrm{ii}}$ & $104.3(5)$ \\
\hline $\mathrm{Ga}^{\mathrm{xxi}}-\mathrm{O} 2-\mathrm{Rb}_{1} \mathrm{~B}^{\mathrm{ii}}$ & $125.9(3)$ \\
\hline $\mathrm{As}-\mathrm{O} 2-\mathrm{Rb} 1 \mathrm{~B}$ & $94.4(5)$ \\
\hline $\mathrm{Ga} 1^{\mathrm{xxiv}}-\mathrm{O} 2-\mathrm{Rb} 1 \mathrm{~B}$ & $130.14(12)$ \\
\hline $\mathrm{Rb} 1 \mathrm{~B}^{\mathrm{ii}}-\mathrm{O} 2-\mathrm{Rb} 1 \mathrm{~B}$ & $11.7(11)$ \\
\hline $\mathrm{As}-\mathrm{O} 2-\mathrm{Rb} 1 \mathrm{~A}$ & $99.78(6)$ \\
\hline $\mathrm{Ga}^{\mathrm{xxiv}}-\mathrm{O} 2-\mathrm{Rb} 1 \mathrm{~A}$ & $128.83(6)$ \\
\hline $\mathrm{Rb} 1 \mathrm{~B}^{\mathrm{ii}}-\mathrm{O} 2-\mathrm{Rb} 1 \mathrm{~A}$ & $4.8(5)$ \\
\hline $\mathrm{Rb} 1 \mathrm{~B}-\mathrm{O} 2-\mathrm{Rb} 1 \mathrm{~A}$ & $7.1(7)$ \\
\hline $\mathrm{As}-\mathrm{O} 2-\mathrm{Rb} 2$ & $60.35(4)$ \\
\hline $\mathrm{Ga}{ }^{\mathrm{xxiv}}-\mathrm{O} 2-\mathrm{Rb} 2$ & $162.84(6)$ \\
\hline $\mathrm{Rb} \mathrm{B}^{\mathrm{ii}}-\mathrm{O} 2-\mathrm{Rb} 2$ & $64.9(2)$ \\
\hline $\mathrm{Rb} 1 \mathrm{~B}-\mathrm{O} 2-\mathrm{Rb} 2$ & $63.47(7)$ \\
\hline $\mathrm{Rb} 1 \mathrm{~A}-\mathrm{O} 2-\mathrm{Rb} 2$ & $63.10(2)$ \\
\hline $\mathrm{As}-\mathrm{O} 3-\mathrm{Rb} 2$ & $129.19(8)$ \\
\hline $\mathrm{As}-\mathrm{O} 3-\mathrm{Rb} 1 \mathrm{~B}$ & $105.64(12)$ \\
\hline $\mathrm{Rb} 2-\mathrm{O} 3-\mathrm{Rb} 1 \mathrm{~B}$ & $97.7(5)$ \\
\hline $\mathrm{As}-\mathrm{O} 3-\mathrm{Rb} 1 \mathrm{~B}^{\mathrm{ii}}$ & $98.2(6)$ \\
\hline $\mathrm{Rb} 2-\mathrm{O} 3-\mathrm{Rb} \mathrm{B}^{\mathrm{ii}}$ & $94.64(16)$ \\
\hline $\mathrm{Rb} 1 \mathrm{~B}-\mathrm{O} 3-\mathrm{Rb} 1 \mathrm{~B}^{\mathrm{ii}}$ & $13.2(13)$ \\
\hline $\mathrm{As}-\mathrm{O} 3-\mathrm{Rb} 1 \mathrm{~A}$ & $104.65(8)$ \\
\hline $\mathrm{Rb} 2-\mathrm{O} 3-\mathrm{Rb} 1 \mathrm{~A}$ & $93.37(5)$ \\
\hline $\mathrm{Rb} 1 \mathrm{~B}-\mathrm{O} 3-\mathrm{Rb} 1 \mathrm{~A}$ & $7.0(7)$ \\
\hline $\mathrm{Rb} 1 \mathrm{~B}^{\mathrm{ii}}-\mathrm{O} 3-\mathrm{Rb} 1 \mathrm{~A}$ & $7.5(7)$ \\
\hline $\mathrm{As}-\mathrm{O} 3-\mathrm{Rb} 1 \mathrm{~B}^{\mathrm{i}}$ & $109.5(4)$ \\
\hline $\mathrm{Rb} 2-\mathrm{O} 3-\mathrm{Rb} \mathrm{B}^{\mathrm{i}}$ & $88.4(5)$ \\
\hline $\mathrm{Rb} 1 \mathrm{~B}-\mathrm{O} 3-\mathrm{Rb} \mathrm{B}^{\mathrm{i}}$ & $10.0(9)$ \\
\hline $\mathrm{Rb}_{1 \mathrm{~B}}{ }^{\mathrm{ii}}-\mathrm{O} 3-\mathrm{Rb} \mathrm{B}^{\mathrm{i}}$ & $11.3(10)$ \\
\hline $\mathrm{Rb} 1 \mathrm{~A}-\mathrm{O} 3-\mathrm{Rb} 1 \mathrm{~B}^{\mathrm{i}}$ & $5.4(5)$ \\
\hline $\mathrm{As}-\mathrm{O} 3-\mathrm{Rb} 2^{\mathrm{xii}}$ & $82.99(7)$ \\
\hline $\mathrm{Rb} 2-\mathrm{O} 3-\mathrm{Rb} 2^{\mathrm{xii}}$ & $96.50(5)$ \\
\hline $\mathrm{Rb} 1 \mathrm{~B}-\mathrm{O} 3-\mathrm{Rb} 2^{\mathrm{xii}}$ & $152.4(7)$ \\
\hline $\mathrm{Rb} 1 \mathrm{~B}^{\mathrm{ii}}-\mathrm{O} 3-\mathrm{Rb} 2^{\mathrm{xii}}$ & $164.5(4)$ \\
\hline $\mathrm{Rb} 1 \mathrm{~A}-\mathrm{O} 3-\mathrm{Rb} 2^{\mathrm{xii}}$ & $159.28(6)$ \\
\hline $\mathrm{Rb} 1 \mathrm{~B}^{\mathrm{i}}-\mathrm{O} 3-\mathrm{Rb} 2^{\mathrm{xii}}$ & $159.23(8)$ \\
\hline $\mathrm{As}{ }^{\mathrm{iv}}-\mathrm{O} 4-\mathrm{Ga} 1^{\mathrm{xxvi}}$ & $130.00(8)$ \\
\hline $\mathrm{As}^{\mathrm{iv}}-\mathrm{O} 4-\mathrm{Rb} 2^{\mathrm{xxvii}}$ & $84.95(5)$ \\
\hline $\mathrm{Ga} 1^{\mathrm{xxvi}}-\mathrm{O} 4-\mathrm{Rb} 2^{\mathrm{xxvii}}$ & $100.50(6)$ \\
\hline $\mathrm{As}^{\mathrm{iv}}-\mathrm{O} 4-\mathrm{Rb} 1 \mathrm{~A}^{\mathrm{xxv}}$ & $124.06(6)$ \\
\hline $\mathrm{Ga} 1^{\mathrm{xxvi}}-\mathrm{O} 4-\mathrm{Rb} 1 \mathrm{~A}^{\mathrm{xxv}}$ & $96.95(5)$ \\
\hline $\mathrm{Rb} 2^{\mathrm{xxvii}}-\mathrm{O} 4-\mathrm{Rb} 1 \mathrm{~A}^{\mathrm{xxv}}$ & $118.51(4)$ \\
\hline
\end{tabular}




\section{supporting information}

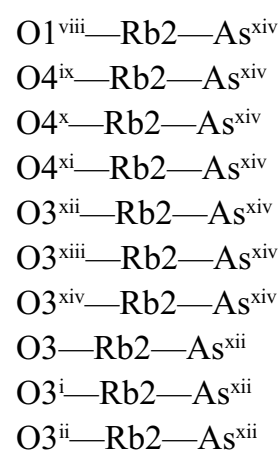

$88.53(3)$

$71.43(3)$

$26.56(2)$

$54.15(3)$

$63.12(3)$

$98.10(3)$

$27.50(3)$

100.79 (4)

102.80 (4)

177.27 (4)
$\mathrm{As}^{\mathrm{iv}}$ - $\mathrm{O} 4-\mathrm{Rb} 1 \mathrm{~A}$

$\mathrm{Ga}^{\mathrm{xxvi}}-\mathrm{O} 4-\mathrm{Rb} 1 \mathrm{~A}$

$\mathrm{Rb} 2^{\text {xxvii }}-\mathrm{O} 4-\mathrm{Rb} 1 \mathrm{~A}$

$\mathrm{Rb} 1 \mathrm{~A}^{\mathrm{xxv}}-\mathrm{O} 4-\mathrm{Rb} 1 \mathrm{~A}$

$\mathrm{As}^{\mathrm{iv}}-\mathrm{O} 4-\mathrm{Rb} 2^{\text {xxviii }}$

$\mathrm{Ga} 1^{\mathrm{xxv}}-\mathrm{O} 4-\mathrm{Rb} 2^{\mathrm{xxvii}}$

$\mathrm{Rb} 2^{\mathrm{xxvii}}-\mathrm{O} 4-\mathrm{Rb} 2^{\text {xxviii }}$

$\mathrm{Rb}_{1} \mathrm{~A}^{\mathrm{xxy}}-\mathrm{O} 4-\mathrm{Rb} 2^{\mathrm{xxviii}}$

$\mathrm{Rb} 1 \mathrm{~A}-\mathrm{O} 4-\mathrm{Rb} 2^{\text {xxviii }}$
$51.95(4)$

$78.99(4)$

$104.39(3)$

$136.81(4)$

$98.27(5)$

$129.76(6)$

$66.64(2)$

$57.20(2)$

$150.18(3)$

Symmetry codes: (i) $-y, x-y, z$; (ii) $-x+y,-x, z$; (iii) $x-y,-y,-z+3 / 2$; (iv) $-x,-x+y,-z+3 / 2$; (v) $y, x,-z+3 / 2$; (vi) $-x+2 / 3,-y-2 / 3,-z+4 / 3$; (vii) $x-y-4 / 3$, $x-2 / 3,-z+4 / 3$; (viii) $y+2 / 3,-x+y+4 / 3,-z+4 / 3$; (ix) $x-1 / 3, x-y-2 / 3, z-1 / 6$; (x) $-y-1 / 3,-x+1 / 3, z-1 / 6$; (xi) $-x+y+2 / 3, y+1 / 3, z-1 / 6$; (xii) $-x-1 / 3,-y-2 / 3$, $-z+4 / 3$; (xiii) $y+2 / 3,-x+y+1 / 3,-z+4 / 3$; (xiv) $x-y-1 / 3, x+1 / 3,-z+4 / 3$; (xv) $-y, x-y+1, z$; (xvi) $x+1, y+1, z$; (xvii) $x, y+1, z$; (xviii) $-x+y+1,-x+1, z$; (xix) $-x+2 / 3,-y+1 / 3,-z+4 / 3$; (xx) $x-1, y, z$; (xxi) $-y+1 / 3,-x+2 / 3, z+1 / 6$; (xxii) $-x-1 / 3,-y+1 / 3,-z+4 / 3$; (xxiii) $-x+2 / 3,-y+4 / 3,-z+4 / 3$; (xxiv) $x-1, y-1, z$; (xxv) $x+1, y, z$; (xxvi) $x, y-1, z$; (xxvii) $-y+1 / 3,-x-1 / 3, z+1 / 6$; (xxviii) $y+1, x,-z+3 / 2$.

Hydrogen-bond geometry $\left(\stackrel{A}{\circ}{ }^{\circ}\right)$

\begin{tabular}{lllll}
\hline$D-\mathrm{H} \cdots A$ & $D-\mathrm{H}$ & $\mathrm{H} \cdots A$ & $D \cdots A$ & $D-\mathrm{H} \cdots A$ \\
\hline $\mathrm{O} 3-\mathrm{H} \cdots{ }^{\text {Oxix }}$ & $0.85(3)$ & $1.76(3)$ & $2.598(2)$ & $168(4)$
\end{tabular}

Symmetry code: (xxix) $y, x-1,-z+3 / 2$. 